\title{
Efficient Revocable Identity-Based Encryption via Subset Difference Methods
}

\author{
Kwangsu Lee* Dong Hoon Lee ${ }^{\dagger} \quad$ Jong Hwan Park
}

\begin{abstract}
Providing an efficient revocation mechanism for identity-based encryption (IBE) is very important since a user's credential (or private key) can be expired or revealed. Revocable IBE (RIBE) is an extension of IBE that provides an efficient revocation mechanism. Previous RIBE schemes essentially use the complete subtree (CS) scheme of Naor, Naor and Lotspiech (CRYPTO 2001) for key revocation. In this paper, we present a new technique for RIBE that uses the efficient subset difference (SD) scheme of Naor et al. instead of using the CS scheme to improve the size of update keys. Following our new technique, we first propose an efficient RIBE scheme in prime-order bilinear groups by combining the IBE scheme of Boneh and Boyen and the SD scheme and prove its selective security under the standard assumption. Our RIBE scheme is the first RIBE scheme in bilinear groups that has $O(r)$ number of group elements in an update key where $r$ is the number of revoked users. Next, we also propose another RIBE scheme in composite-order bilinear groups and prove its full security under static assumptions. Our RIBE schemes also can be integrated with the layered subset difference (LSD) scheme of Halevy and Shamir (CRYPTO 2002) to reduce the size of a private key.
\end{abstract}

Keywords: Identity-based encryption, Revocable identity-based encryption, Key revocation, Subset difference method, Bilinear maps.

\footnotetext{
*Sejong University, Korea. Email: kwangsu@ sejong.ac.kr. This work was partially done at Korea University.

${ }^{\dagger}$ Korea University, Korea. Email: donghlee@korea. ac. kr.

† Sangmyung University, Korea. Email: jhpark@smu. ac.kr.
} 


\section{Introduction}

Identity-based encryption (IBE) is a new paradigm of public-key encryption (PKE) that uses the identity string of a user for the public key of the user [5, 6, 40, 42]. IBE and its extensions like hierarchical IBE (HIBE) [5, 12, 16], attribute-based encryption (ABE) [3, 14, 37], predicate encryption (PE) [8, 17, 21], and functional encryption (FE) [7, 13] opened new applications of encryption systems such as the delegation of decryption capability, access control in encrypted data, searches on encrypted data, and functional evaluation on encrypted data. If an IBE scheme is used in real-world applications, an efficient revocation mechanism for IBE that can handle dynamic credentials of users is needed since a user's credential can be revealed or expired. Revocable IBE (RIBE) is an extension of IBE that can handle the dynamic credentials of users by providing an efficient revocation mechanism. An ideal revocation method in IBE is that a sender just creates a ciphertext without worrying about the revocation of a receiver and only the receiver needs to check the revocation of his credential to decrypt the ciphertext.

Boneh and Franklin [6] proposed the first RIBE scheme by representing an identity as $I D \| T$ where $I D$ is the real identity and $T$ is a current time, but it is inefficient and not scalable because all non-revoked users should update their private keys from a trusted center by establishing secure channels. A scalable RIBE scheme was introduced by Boldyreva, Goyal, and Kumar [4]. They defined the first formal definition of RIBE and proposed a selectively secure RIBE scheme by combining the fuzzy IBE (FIBE) scheme of Sahai and Waters [37] and the complete subtree (CS) scheme of Naor et al. [29]. In their RIBE scheme, each user receives a (long-term) private key $S K_{I D}$ for an identity $I D$ from a trusted center where $S K_{I D}$ is associated with path nodes in a binary tree, and the center periodically broadcasts an update key $U K_{T, R}$ on current time $T$ for non-revoked users that are not in $R$ where $U K_{T, R}$ is associated with minimum covering subsets in the binary tree. If a user with $S K_{I D}$ is not revoked in $R$ on time $T$, then he can drive a (short-term) decryption key $D K_{I D, T}$ from $S K_{I D}$ and $U K_{T, R}$ to decrypt a ciphertext $C T_{I D, T}$. After the work of Boldyreva et al., fully secure RIBE schemes [26, 39], a revocable HIBE (RHIBE) scheme [38], and revocable-storage ABE (RS-ABE) schemes [18,36] were proposed.

Although efficient RIBE schemes and their extended schemes were proposed, the main design principle of these constructions essentially follows that of Boldyreva et al. [4] that uses the CS scheme of Naor et al. [29] for key revocation. The CS scheme is one instance of the general subset cover framework of Naor et al. and there are other efficient subset cover schemes like the subset difference (SD) scheme [29] and the layered subset difference (LSD) scheme [15]. Therefore, we ask the following natural question in this paper.

"Can we build an efficient RIBE scheme by using the SD scheme (or the LSD scheme) instead of using the CS scheme?"

If it is possible, then the size of an update key can be reduced from $O\left(r \log \left(N_{\max } / r\right)\right)$ to $O(r)$ group elements by slightly increasing the size of a private key where $N_{\max }$ is the maximum number of users and $r$ is the number of revoked users. Reducing the size of an update key is an important issue in RIBE since an update key should be broadcasted to all users for each time period whereas a private key is given to a user at the first time only.

\subsection{Our Results}

In this paper, we give the affirmative answer for the above question by presenting a new technique that combines an IBE scheme and the SD scheme. The followings are our results:

New Technique for RIBE. We first present a new technique for RIBE that combines an IBE scheme and the SD scheme instead of using the CS scheme. The CS scheme was easily integrated with an IBE scheme 
since an assigned key for a subset in the CS scheme is independent of each other [29]. However, it is unclear how to integrate the SD scheme with an IBE scheme since an assigned key for a subset in the SD scheme is dependent on other keys [29]. We use a random polynomial of degree one to solve the complex key assignment problem in the SD scheme, and then integrate the SD scheme with an IBE scheme by using the observation that the SD scheme is related with a single member revocation scheme which can be implemented by a random polynomial of degree one. However, this idea does not directly lead to a secure scheme because of collusion attacks. To provide the security against collusion attacks, we personalize the private key components with an identity $I D$ and constrain the usage of update key components by time $T$. The technical details are discussed below in this section.

RIBE with Selective Security. Following our new technique for RIBE, we construct an RIBE scheme in prime-order bilinear groups by combining the IBE scheme of Boneh and Boyen [5] and the SD scheme of Naor et al. [29], and then we prove its security in the selective revocation list model under the standard assumption. In our RIBE scheme, the number of group elements in the public parameters, a private key, an update key, and a ciphertext is $O(1), O\left(\log ^{2} N_{\text {max }}\right), O(r)$, and $O(1)$ respectively where $N_{\text {max }}$ is the maximum number of users and $r$ is the number of revoked users. Compared with the previous RIBE schemes [4, 26, 39] that have $O\left(r \log \left(N_{\max } / r\right)\right)$ group elements in an update key, our RIBE scheme just has $O(r)$ group elements in an update key. If the LSD scheme of Halevy and Shamir [15] is used instead of the SD scheme, then the number of group elements in a private key is reduced from $O\left(\log ^{2} N_{\max }\right)$ to $O\left(\log ^{1.5} N_{\max }\right)$. The detailed comparison of RIBE schemes is given in Table1.

RIBE with Full Security. Next, we propose another RIBE scheme in composite-order bilinear groups by combining the IBE scheme of Lewko and Waters [25] and the SD scheme, and then we prove its security in the full model under static assumptions. To prove the security in the full model, we use the dual system encryption technique of Waters [25,43]. However, the original dual system encryption technique that was used to prove the security of IBE and HIBE is not directly applicable to an RIBE scheme since the adversary of RIBE can request a private key for a challenge identity $I D^{*}$ and an update key for a challenge time $T^{*}$ that were not allowed in the security model of IBE. Additionally, the complex key assignment of the SD scheme introduces another difficulties in the proof of using the dual system encryption technique. To solve these problems, we carefully redesign the semi-functional types of each key and hybrid games for the dual system encryption.

\subsection{Our Techniques}

The CS scheme can be easily integrated with an IBE scheme to construct an RIBE scheme since it assigns a random independent key for each subset in CS [4,26,39]. That is, the master key $g^{\alpha}$ of RIBE is split into two shares $g^{\gamma_{i}}$ and $g^{\alpha-\gamma_{i}}$ to build a private key and an update key respectively where $\gamma_{i}$ is a random independent key for a subset $S_{i}$, and these shares are easily reconstructed as $g^{\alpha}=g^{\gamma_{i}} \cdot g^{\alpha-\gamma_{i}}$. In contrast, the SD scheme cannot be easily integrated with an IBE scheme to construct an RIBE scheme since it assigns a dependent key for each subset in SD by using a pseudo-random generator [29]. That is, if a dependent key generated by a pseudo-random generator is raised to an exponent, then the dependency between group elements cannot be computed without solving the discrete logarithm problem. In the SD scheme, a subset $S_{i, j}$ that is associated with two nodes $v_{i}$ and $v_{j}$ of a binary tree is defined as the set of leaf nodes that belong to $T_{i} \backslash T_{j}$ where $T_{i}$ is a subtree rooted at $v_{i}$ and $T_{j}$ is a subtree rooted at $v_{j}$. To overcome the complex and dependent key assignment of the SD scheme by using a pseudo-random generator, we use the observation that a subset $S_{i, j}$ in SD can be interpreted as single member revocation. That is, if we define a group label $G L$ as the set of subsets $S_{i, j}$ such that $v_{i}$ is the same and the depth $d_{j}$ of $v_{j}$ is also the same, then the subset $S_{i, j}$ can be interpreted as this subset 
Table 1: Comparison of revocable identity-based encryption schemes

\begin{tabular}{lccccccc}
\hline Scheme & PP Size & SK Size & UK Size & CT Size & Decrypt Cost & Model & Assumption \\
\hline BF [6] & $1 k_{p}$ & $1 k_{p}$ & $\left(N_{\max }-r\right) k_{p}$ & $2 k_{p}$ & $1 \mathrm{P}$ & $\mathrm{RO}, \mathrm{AD}$ & $\mathrm{BDH}$ \\
$\mathrm{BGK}[4]$ & $5 k_{p}$ & $2 \log \left(N_{\max }\right) k_{p}$ & $2 r \log \left(N_{\max } / r\right) k_{p}$ & $4 k_{p}$ & $4 \mathrm{P}+4 \mathrm{E}$ & $\mathrm{SE}$ & $\mathrm{DBDH}$ \\
LV [26] & $2 \lambda k_{p}$ & $3 \log \left(N_{\max }\right) k_{p}$ & $3 r \log \left(N_{\max } / r\right) k_{p}$ & $5 k_{p}$ & $3 \mathrm{P}+7 \mathrm{E}$ & $\mathrm{AD}$ & $\mathrm{mDBDH}$ \\
$\mathrm{SE}[39]$ & $2 \lambda k_{p}$ & $2 \log \left(N_{\max }\right) k_{p}$ & $2 r \log \left(N_{\max } / r\right) k_{p}$ & $4 k_{p}$ & $3 \mathrm{P}+6 \mathrm{E}$ & $\mathrm{AD}$ & $\mathrm{DBDH}$ \\
Ours (SD) & $-5 k_{p}$ & $\log ^{2}\left(N_{\max }\right) k_{p}$ & $2 r k_{p}$ & $4 k_{p}$ & $3 \mathrm{P}+10 \mathrm{E}$ & $\mathrm{SRL}$ & $\mathrm{DBDH}$ \\
Ours (SD) & $6 k_{c}$ & $\log ^{2}\left(N_{\max }\right) k_{c}$ & $2 r k_{c}$ & $4 k_{c}$ & $3 \mathrm{P}+10 \mathrm{E}$ & $\mathrm{AD}$ & Static \\
Ours (LSD) & $5 k_{p}$ & $\log ^{1.5}\left(N_{\max }\right) k_{p}$ & $4 r k_{p}$ & $4 k_{p}$ & $3 \mathrm{P}+10 \mathrm{E}$ & $\mathrm{SRL}$ & $\mathrm{DBDH}$ \\
Ours (LSD) & $6 k_{c}$ & $\log ^{1.5}\left(N_{\max }\right) k_{c}$ & $4 r k_{c}$ & $4 k_{c}$ & $3 \mathrm{P}+10 \mathrm{E}$ & $\mathrm{AD}$ & Static \\
\hline
\end{tabular}

We let $\lambda$ be a security parameter, $N_{\max }$ be the number of maximum users, $r$ be the number of revoked users, $k_{p}\left(k_{c}\right)$ be the element bit size of prime-order (composite-order) groups. For security model, we use symbols RO for random oracle model, AD for adaptive model, SE for selective model, and SRL for selective revocation list model. We also use symbols $\mathrm{P}$ for a pairing operation and $\mathrm{E}$ for an exponentiation.

$S_{i, j}$ is almost the same as $G L$ except that one member node $v_{j}$ is excluded (or revoked). This observation was implicitly made by Lee et al. [20] and they used this observation to construct a public-key trace and revoke scheme by combining the SD scheme and a single-revocation encryption scheme in bilinear groups (without using a pseudo-random generator). Unfortunately, the technique of Lee et al. cannot be used to construct an RIBE scheme since two key shares should be reconstructed without using bilinear map operations in RIBE. Alternatively, we employ the polynomial-based revocation scheme of Naor and Pinkas [31]. That is, a revocation scheme that uses a random polynomial of degree one can be used to revoke a single user, and the polynomial-based secret sharing scheme works well in exponents because of the linear reconstruction property.

In the SD scheme, a collection $S$ is defined as the set of subsets $S_{i, j}$ where $v_{i}$ and $v_{j}$ is a node in a tree and $v_{j}$ is a descendant of $v_{i}$. As mentioned before, each subset $S_{i, j}$ can be categorized as a group label and one group label GL is defined as the set of subsets $S_{i, j}$ such that $v_{i}$ is the same and the depth $d_{j}$ of $v_{j}$ is the same. To use the polynomial-based revocation scheme, we assign a random polynomial $f_{G L}(x)=a_{G L} x+\alpha$ once to each group label where $a_{G L}$ is a random value and $\alpha$ is a fixed value for all group labels. In a group label $G L$, each member $L_{j}$ that is associated with a node $v_{j}$ has a share $g^{f_{G L}\left(L_{j}\right)}$ where $L_{j}$ is the identifier of the node $v_{j}$. If one member $L_{j^{\prime}}$ is revoked, then his share $g^{f_{G L}\left(L_{j^{\prime}}\right)}$ is revealed to all members. Thus any member in the group label $G L$ except the revoked member can reconstruct the secret $g^{\alpha}$ by using the Lagrange interpolation method since two points of a degree one polynomial are enough for reconstruction. However, this simple method is insecure against collusion attacks since any two members can collude to reconstruct the secret $g^{\alpha}$.

To provide the security against collusion attacks, the share of a member is personalized by using his identity $I D$ and the share of the revoked member is constrained by using revoked time $T$. That is, a personalized private key for a member $L_{j}$ is defined as $g^{f_{G L}\left(L_{j}\right)} H(I D)^{r_{1}}, g^{r_{1}}$ where $H$ is a hash function and a time-constrained update key for a revoked member $L_{j^{\prime}}$ is defined as $g^{f_{G L}\left(L_{j}^{\prime}\right)} H(T)^{r_{2}}, g^{-r_{2}}$. Thus a nonrevoked member in the group label can derive a decryption key as $g^{\alpha} H(I D)^{r_{1}} H(T)^{r_{2}}, g^{-r_{1}}, g^{-r_{2}}$ from his personalized private key and the time-constrained update key. Note that if two revoked members collude, then they only can derive $g^{\alpha} H(I D)^{r_{1}} H\left(I D^{\prime}\right)^{r_{2}}, g^{-r_{1}}, g^{-r_{2}}$ that are not useful to decrypt a ciphertext. In the 
RIBE scheme, a private key for a user consists of many subsets and an update key for a time period also consists of many subsets.

\subsection{Related Work}

Certificate Revocation in PKE. In PKE that uses public-key infrastructure (PKI), certificate revocation list (CRL) and online certificate status protocol (OCSP) are traditional methods to revoke certificates of users. However, these methods are inefficient in terms of transmission costs and computation costs since CRL includes all serial numbers of revoked certificates and OCSP requires the generation of digital signature for each queries. Furthermore, they also require for each client who uses a certificate to implement a path validation module to check the validity of a digital signature in CRL or OCSP. A better solution named certificate revocation system (CRS) was proposed by Micali [28] and it uses a hash-chain to check the validity of the certificate. This method was improved by Naor and Nissim [30] and Aiello et al. [1]. Although CRS improves the previous CRL and OCSP, these methods still require a sender to check the validity of a certificate through a heavy infrastructure and this problem is the serious point of these methods. Gentry [11] solved this problem by introducing certificate-based encryption (CBE) and proposed an efficient $\mathrm{CBE}$ scheme in bilinear groups.

Revocation in IBE. As mentioned, an ideal revocation method for IBE is that a sender can create a ciphertext as the same as that of IBE without worrying about the revocation of a receiver and only the receiver checks the revocation of his key to decrypt the ciphertext. Boneh and Franklin [6] proposed the first IBE scheme that supports the revocation capability, but their scheme is inefficient and not scalable since each user should be connected to the center through a secure channel to receive an updated private key. A scalable and RIBE scheme was proposed by Boldyreva et al. [4]. They constructed an RIBE scheme by combining the FIBE scheme of Sahai and Waters [37] and the CS scheme, and then proved its selective security. After that, fully secure RIBE schemes were proposed by Libert and Vergnaud [26] and Seo and Emura [39] where Seo and Emura refined the security model of RIBE by considering the decryption key exposure attacks. Recently, Park et al. [35] proposed an RIBE scheme with shorter private keys and update keys by using multilinear maps, but the size of the public parameters is dependent to the number of all users. The design technique of RIBE also can be applicable to the extensions of IBE, like HIBE, ABE, and PE. Boldyreva et al. [4] already proposed a revocable ABE (R-ABE) scheme. Seo and Emura [38] proposed an RHIBE scheme by using the HIBE scheme of Boneh and Boyen. For cloud storage, Sahai et al. [36] proposed RS-ABE schemes that provide the key revocation and ciphertext update functionalities, and Lee et al. [18] proposed improved RS-ABE and RS-PE schemes by introducing self-updatable encryption.

Revocation Encryption. Revocation encryption (RE) is a special type of broadcast encryption (BE) [10], in which a sender creates a ciphertext by specifying the set of revoked users $R$ instead of specifying the set of receivers $S$ and a receiver can decrypt the ciphertext if he is not included in $R$ [24,29,31]. However, there is a crucial difference between the security model of RE and that of RIBE. In RE, the collusion of nonrevoked users is not allowed since an adversary cannot request private keys for non-revoked users [24], but the collusion of non-revoked users is allowed in RIBE since an adversary can request private keys for nonrevoked users except the challenge user $I D^{*}[4]$. Although $\mathrm{RE}$ alone does not directly solve the revocation problem of IBE, RE can be combined with IBE or its extensions to directly revoke the set of revoked users by specifying a receiver and a revoked set $R$ in a ciphertext [2, 19,32, 41] . In this approach, a sender should take care of the revocation of users. 


\section{Preliminaries}

In this section, we introduce the subset difference method and define the syntax and the security model of revocable IBE.

\subsection{Full Binary Tree}

A full binary tree $\mathcal{B} \mathcal{T}$ is a tree data structure where all internal nodes have two child nodes and all leaf nodes have the same depth. Let $N_{\max }$ be the number of leaf nodes in $\mathcal{B T}$. The number of all nodes in $\mathcal{B T}$ is $2 N_{\max }-1$ and for any $1 \leq i \leq 2 N_{\max }-1$ we denote by $v_{i}$ a node in $\mathcal{B T}$. The depth $d_{i}$ of a node $v_{i}$ is the length of the path from the root node to the node. The root node is at depth zero. The depth of $\mathcal{B} \mathcal{T}$ is the length of the path from the root node to a leaf node. A level of $\mathcal{B} \mathcal{T}$ is a set of all nodes at given depth. For any node $v_{i} \in \mathcal{B} \mathcal{T}, T_{i}$ is defined as a subtree that is rooted at $v_{i}$. For any two nodes $v_{i}, v_{j} \in \mathcal{B} \mathcal{T}$ where $v_{j}$ is a descendant of $v_{i}, T_{i, j}$ is defined as a subtree $T_{i}-T_{j}$, that is, all nodes that are descendants of $v_{i}$ but not $v_{j}$. For any node $v_{i} \in \mathcal{B T}, S_{i}$ is defined as the set of leaf nodes in $T_{i}$. Similarly, $S_{i, j}$ is defined as the set of leaf nodes in $T_{i, j}$, that is, $S_{i, j}=S_{i} \backslash S_{j}$.

For any node $v_{i} \in \mathcal{B} \mathcal{T}, L_{i}$ is defined as an identifier that is a fixed and unique string. The identifier of each node in the tree is assigned as follows: Each edge in the tree is assigned with 0 or 1 depending on whether the edge is connected to its left or right child node. The identifier $L_{i} \in\{0,1\}^{*}$ of a node $v_{i}$ is defined as the bitstring obtained by reading all the labels of edges in the path from the root node to the node $v_{i}$. We define $\mathbf{L a b e l}\left(S_{i, j}\right)$ as a function that uniquely maps a subset $S_{i, j}$ to identifiers $\left(L_{i}, L_{j}\right)$ where $L_{i}$ and $L_{j}$ are the identifiers of $v_{i}$ and $v_{j}$ respectively. We also define Depth $\left(S_{j}\right)$ as a function that returns the depth $d_{j}$ of the node $v_{j}$ associated to $S_{j}$.

For a full binary tree $\mathcal{B T}$ and a subset $R$ of leaf nodes, $S T(\mathcal{B T}, R)$ is defined as the Steiner Tree induced by the set $R$ and the root node, that is, the minimal subtree of $\mathcal{B} \mathcal{T}$ that connects all the leaf nodes in $R$ and the root node. we simply denote $S T(\mathcal{B} \mathcal{T}, R)$ by $S T(R)$.

\subsection{Subset Difference Method}

The subset difference (SD) method is a special instance of the subset cover framework of Naor, Naor, and Lotspiech [29] that is a general methodology for revocation schemes. The well-known complete subtree (CS) scheme is also one instance of the subset cover framework. The original subset cover framework consists of a subset assignment part and a key assignment part. In this paper, we define the subset cover framework by using the subset assignment part only. The formal definition is given as follows:

Definition 2.1 (Subset Cover). A subset cover (SC) scheme for the set $\mathcal{N}=\left\{1, \ldots, N_{\max }\right\}$ of users consists of four PPT algorithms Setup, Assign, Cover, and Match, which are defined as follows:

$\operatorname{Setup}\left(N_{\max }\right)$. The setup algorithm takes as input the maximum number $N_{\max }$ of users and outputs a collection $\mathcal{S}$ of subsets $S_{1}, \ldots, S_{w}$ where $S_{i} \subseteq \mathcal{N}$.

$\operatorname{Assign}(\mathcal{S}, u)$. The assigning algorithm takes as input the collection $\mathcal{S}$ and a user $u \in \mathcal{N}$, and outputs a private set $P V_{u}=\left\{S_{j_{1}}, \ldots, S_{j_{n}}\right\}$ that is associated with the user $u$.

$\operatorname{Cover}(\mathcal{S}, R)$. The covering algorithm takes as the collection $\mathcal{S}$ and a revoked set $R \subset \mathcal{N}$ of users, and outputs a covering set $C V_{R}=\left\{S_{i_{1}} \ldots, S_{i_{m}}\right\}$ that is a partition of the non-revoked users $\mathcal{N} \backslash R$ into disjoint subsets $S_{i_{1}}, \ldots, S_{i_{m}}$ where $\mathcal{N} \backslash R=\bigcup_{k=1}^{m} S_{i_{k}}$. 
$\operatorname{Match}\left(C V_{R}, P V_{u}\right)$. The matching algorithm takes as input a covering set $C V_{R}=\left\{S_{i_{1}}, \ldots, S_{i_{m}}\right\}$ and a private set $P V_{u}=\left\{S_{j_{1}}, \ldots, S_{j_{n}}\right\}$ of a user $u$. It outputs $\left(S_{i_{k}}, S_{j_{k^{\prime}}}\right)$ such that $S_{i_{k}} \in C V_{R}, u \in S_{i_{k}}$, and $S_{j_{k^{\prime}}} \in P V_{u}$, or it outputs $\perp$.

The correctness of SC is defined as follows: For all $\mathcal{S}$ generated by Setup, all $P V_{u}$ generated by Assign, and any $R$, it is required that:

- If $u \notin R$, then $\operatorname{Match}\left(\operatorname{Cover}(\mathcal{S}, R), P V_{u}\right)=\left(S_{i_{k}}, S_{j_{k^{\prime}}}\right)$ such that $S_{i_{k}} \in C V_{R}$ and $S_{j_{k^{\prime}}} \in P V_{u}$.

- If $u \in R$, then $\operatorname{Match}\left(\operatorname{Cover}(\mathcal{S}, R), P V_{u}\right)=\perp$.

Note that the exact conditions of the subsets outputted by the matching algorithm is defined by the specific instance of the SC scheme.

As mentioned, the SD scheme is one instance of the SC scheme and it was proposed by Naor et al. [29] as an improvement on the CS scheme. The SD scheme is described as follows:

SD.Setup $\left(N_{\max }\right)$ : This algorithm takes as input the maximum number $N_{\max }$ of users. Let $N_{\max }=2^{n}$ for simplicity. It first sets a full binary tree $\mathcal{B} \mathcal{T}$ of depth $n$. Each user is assigned to a different leaf node in $\mathcal{B T}$. The collection $\mathcal{S}$ of SD is the set of all subsets $\left\{S_{i, j}\right\}$ where $v_{i}, v_{j} \in \mathcal{B T}$ and $v_{j}$ is a descendant of $v_{i}$. It outputs the full binary tree $\mathcal{B} \mathcal{T}$.

$\operatorname{SD.Assign}(\mathcal{B T}, u)$ : This algorithm takes as input the tree $\mathcal{B T}$ and a user $u \in \mathcal{N}$. Let $v_{u}$ be the leaf node of $\mathcal{B} \mathcal{T}$ that is assigned to the user $u$. Let $\left(v_{k_{0}}, v_{k_{1}}, \ldots, v_{k_{n}}\right)$ be the path from the root node $v_{k_{0}}$ to the leaf node $v_{k_{n}}=v_{u}$. It first sets a private set $P V_{u}$ as an empty one. For all $i, j \in\left\{k_{0}, k_{1}, \ldots, k_{n}\right\}$ where $v_{j}$ is a descendant of $v_{i}$, it adds the subset $S_{i, j}$ defined by two nodes $v_{i}$ and $v_{j}$ in the path into $P V_{u}$. It outputs the private set $P V_{u}=\left\{S_{i, j}\right\}$.

SD.Cover $(\mathcal{B} \mathcal{T}, R)$ : This algorithm takes as input the tree $\mathcal{B} \mathcal{T}$ and a revoked set $R$ of users. It first sets a subtree $T$ as $S T(R)$, and then it builds a covering set $C V_{R}$ iteratively by removing nodes from $T$ until $T$ consists of just a single node as follows:

1. It finds two leaf nodes $v_{i}$ and $v_{j}$ in $T$ where the least-common-ancestor $v$ of $v_{i}$ and $v_{j}$ does not contain any other leaf nodes of $T$ in its subtree. Let $v_{l}$ and $v_{k}$ be the two child nodes of $v$ where $v_{i}$ is a descendant of $v_{l}$ and $v_{j}$ is a descendant of $v_{k}$. If there is only one leaf node left, it makes $v_{i}=v_{j}$ to the leaf node, $v$ to be the root of $T$ and $v_{l}=v_{k}=v$.

2. If $v_{l} \neq v_{i}$, then it adds the subset $S_{l, i}$ to $C V_{R}$; likewise, if $v_{k} \neq v_{j}$, it adds the subset $S_{k, j}$ to $C V_{R}$.

3. It removes from $T$ all the descendants of $v$ and makes $v$ a leaf node.

It outputs the covering set $C V_{R}=\left\{S_{i, j}\right\}$.

SD.Match $\left(C V_{R}, P V_{u}\right)$ : This algorithm takes input as a covering set $C V_{R}=\left\{S_{i, j}\right\}$ and a private set $P V_{u}=$ $\left\{S_{i, j}^{\prime}\right\}$. It finds two subsets $S_{i, j}$ and $S_{i^{\prime}, j^{\prime}}^{\prime}$ such that $S_{i, j} \in C V_{R}, S_{i^{\prime}, j^{\prime}}^{\prime} \in P V_{u}$, and $\left(v_{i}=v_{i}^{\prime}\right) \wedge\left(d_{j}=\right.$ $\left.d_{j^{\prime}}\right) \wedge\left(v_{j} \neq v_{j^{\prime}}\right)$ where $d_{j}$ is the depth of $v_{j}$. If it found two subsets, then it outputs $\left(S_{i, j}, S_{i^{\prime}, j^{\prime}}^{\prime}\right)$. Otherwise, it outputs $\perp$.

Lemma 2.1 ( [29]). Let $N_{\text {max }}$ be the number of leaf nodes in a full binary tree and $r$ be the size of a revoked set. In the SD scheme, the size of a private set is $O\left(\log ^{2} N_{\text {max }}\right)$ where the hidden constant is $1 / 2$ and the size of a covering set is at most $2 r-1$. 
Remark 1. The covering algorithm of the SD scheme is only defined for $r \geq 1$ where $r$ is the size of $R$. One simple way to handle the case $r=0$ is to define a new set $R^{\prime}$ by adding a dummy user that is always revoked since $\left|R^{\prime}\right|=r^{\prime}=r+1$. In the SD scheme, the size of the covering set is at most $2 r-1$, but it is rough worst-case analysis and the size is always smaller than that of the CS scheme since a subset in the CS scheme is defined by a subset in the SD scheme [29]. The better analysis of this covering set size is given by Martin et al. [27].

The layered subset difference (LSD) scheme was proposed by Halevy and Shamir [15] to reduce the size of a private set in the SD scheme. The SD scheme in a cryptosystem generally can be replaced by the LSD scheme since the LSD scheme is a special case of the SD scheme.

Lemma 2.2 ([15]). Let $N_{\max }$ be the number of leaf nodes in a full binary tree and $r$ be the size of a revoked set. In the LSD scheme, the size of a private set is $O\left(\log 1.5 N_{\text {max }}\right)$ where the hidden constant is $1 / 2$ and the size of a covering set is at most $4 r-2$.

\subsection{Revocable Identity-Based Encryption}

Revocable IBE (RIBE) is an extension of IBE that can revoke a users if his credential is expired or revealed [4]. In RIBE, a sender creates a ciphertext for a receiver identity $I D$ and time $T$. A user first obtains a (long-term) private key $S K_{I D}$ for his identity $I D$ from a center, and the center periodically broadcasts an update key $U K_{T, R}$ for time $T$ and a revoked identity set $R$. If a user $I D$ is not revoked in $R$ of the update key, then he can derive a (short-term) decryption key $D K_{I D, T}$ for his identity $I D$ and the time $T$ from $S K_{I D}$ and $U K_{T, R}$ and he can use this decryption key to decrypt the ciphertext. Note that the center does not encrypt an update key for broadcasting. The syntax of RIBE is formally defined as follows:

Definition 2.2 (Revocable IBE). A revocable IBE (RIBE) scheme that is associated with the identity space $\mathcal{I}$, the time space $\mathcal{T}$, and the message space $\mathcal{M}$, consists of seven algorithms Setup, GenKey, UpdateKey, DeriveKey, Encrypt, Decrypt, and Revoke, which are defined as follows:

$\operatorname{Setup}\left(1^{\lambda}, N_{\max }\right)$ : The setup algorithm takes as input a security parameter $1^{\lambda}$ and the maximum number of users $N_{\max }$. It outputs a master key $M K$, an (empty) revocation list $R L$, a state $S T$, and public parameters $P P$.

$\operatorname{GenKey}(I D, M K, S T, P P)$ : The private key generation algorithm takes as input an identity $I D \in \mathcal{I}$, the master key $M K$, the state $S T$, and public parameters $P P$. It outputs a private key $S K_{I D}$ for $I D$ and an updated state $S T$.

$\operatorname{UpdateKey}(T, R L, M K, S T, P P):$ The update key generation algorithm takes as input update time $T \in \mathcal{T}$, the revocation list $R L$, the master key $M K$, the state $S T$, and the public parameters $P P$. It outputs an update key $U K_{T, R}$ for $T$ and $R$ where $R$ is a revoked identity set on the time $T$.

DeriveKey $\left(S K_{I D}, U K_{T, R}, P P\right)$ : The decryption key derivation algorithm takes as input a private key $S K_{I D}$, an update key $U K_{T, R}$, and the public parameters $P P$. It outputs a decryption key $D K_{I D, T}$ or $\perp$.

$\operatorname{Encrypt}(I D, T, M, P P)$ : The encryption algorithm takes as input an identity $I D \in \mathcal{I}$, time $T$, a message $M \in \mathcal{M}$, and the public parameters $P P$. It outputs a ciphertext $C T_{I D, T}$ for $I D$ and $T$.

$\operatorname{Decrypt}\left(C T_{I D, T}, D K_{I D^{\prime}, T^{\prime}}, P P\right)$ : The decryption algorithm takes as input a ciphertext $C T_{I D, T}$, a decryption key $D K_{I D^{\prime}, T^{\prime}}$, and the public parameters $P P$. It outputs an encrypted message $M$ or $\perp$. 
$\operatorname{Revoke}(I D, T, R L, S T)$ : The revocation algorithm takes as input an identity $I D$ to be revoked and revocation time $T$, a revocation list $R L$, and a state $S T$. It outputs an updated revocation list $R L$.

The correctness of RIBE is defined as follows: For all $M K, R L, S T$, and $P P$ generated by $\operatorname{Setup}\left(1^{\lambda}, N_{\max }\right)$, $S K_{I D}$ generated by $\operatorname{GenKey}(I D, M K, S T, P P)$ for any $I D, U K_{T, R}$ generated by $\operatorname{UpdateKey}(T, R L, M K, S T, P P)$ for any $T$ and $R L, C T_{I D_{c}, T_{c}}$ generated by $\operatorname{Encrypt}\left(I D_{c}, T_{c}, M, P P\right)$ for any $I D_{c}, T_{c}$, and $M$, it is required that

- If $(I D \notin R)$, then DeriveKey $\left(S K_{I D}, U K_{T, R}, P P\right)=D K_{I D, T}$.

- If $(I D \in R)$, then DeriveKey $\left(S K_{I D}, U K_{T, R}, P P\right)=\perp$ with all but negligible probability.

- If $\left(I D_{c}=I D\right) \wedge\left(T_{c}=T\right)$, then $\operatorname{Decrypt}\left(C T_{I D_{c}, T_{c}}, D K_{I D, T}, P P\right)=M$.

- If $\left(I D_{c} \neq I D\right) \vee\left(T_{c} \neq T\right)$, then $\operatorname{Decrypt}\left(C T_{I D_{c}, T_{c}}, D K_{I D, T}, P P\right)=\perp$ with all but negligible probability.

The security model of RIBE was introduced by Boldyreva et al. [4] and it was refined by Seo and Emura [39] by considering the decryption key exposure attacks. In this paper, we follow the refined security model of RIBE. In the security game of RIBE, an adversary adaptively request a private key for an identity $I D$, an update key for time $T$ and a current revocation list $R L$, a decryption key for an identity $I D$ and time $T$, and a revocation of an identity. In the challenge step, the adversary submits a challenge identity $I D^{*}$, challenge time $T^{*}$, and challenge messages $M_{0}^{*}, M_{1}^{*}$ with additional restrictions and he receives a challenge ciphertext $C T^{*}$ that is an encryption of a message $M_{\mu}^{*}$ for a random bit $\mu$. After that, the adversary may request additional private key, update key, and decryption key queries, and finally he outputs a guess $\mu^{\prime}$. If his guess is correct, then he wins the game. The security of RIBE is formally defined as follows:

Definition 2.3 (Security). The security of RIBE under chosen plaintext attacks is defined in terms of the following experiment between a challenger $\mathcal{C}$ and a PPT adversary $\mathcal{A}$ :

1. Setup: $\mathcal{C}$ generates a master key $M K$, a revocation list $R L$, a state $S T$, and public parameters $P P$ by running $\operatorname{Setup}\left(1^{\lambda}, N_{\text {max }}\right)$. It keeps $M K, R L, S T$ to itself and gives $P P$ to $\mathcal{A}$.

2. Phase 1: $\mathcal{A}$ adaptively request a polynomial number of queries. These queries are processed as follows:

- If this is a private key query for an identity $I D$, then it gives the corresponding private key $S K_{I D}$ to $\mathcal{A}$ by running $\operatorname{GenKey}(I D, M K, S T, P P)$.

- If this is an update key query for time $T$, then it gives the corresponding update key $U K_{T, R}$ to $\mathcal{A}$ by running UpdateKey $(T, R L, M K, S T, P P)$.

- If this is a decryption key query for an identity $I D$ and time $T$, then it gives the corresponding decryption key $D K_{I D, T}$ to $\mathcal{A}$ by running DeriveKey $\left(S K_{I D}, U K_{T, R}, P P\right)$.

- If this is a revocation query for an identity $I D$ and revocation time $T$, then it updates the revocation list $R L$ by running $\operatorname{Revoke}(I D, T, R L, S T)$ with the restriction: The revocation query for time $T$ cannot be queried if the update key query for the time $T$ was already requested.

Note that we assume that the update key queries and the revocation queries are requested in nondecreasing order of time.

3. Challenge: $\mathcal{A}$ submits a challenge identity $I D^{*}$, challenge time $T^{*}$, and two challenge messages $M_{0}^{*}, M_{1}^{*}$ with equal length with the following restrictions: 
- If a private key query for an identity $I D$ such that $I D=I D^{*}$ was requested, then the identity $I D^{*}$ should be revoked at some time $T$ such that $T \leq T^{*}$.

- The decryption key query for $I D^{*}$ and $T^{*}$ was not requested.

$\mathcal{C}$ flips a random coin $\mu \in\{0,1\}$ and gives the challenge ciphertext $C T^{*}$ to $\mathcal{A}$ by running $\operatorname{Encrypt}\left(I D^{*}\right.$, $\left.T^{*}, M_{\mu}^{*}, P P\right)$.

4. Phase 2: $\mathcal{A}$ may continue to request a polynomial number of additional queries subject to the same restrictions as before.

5. Guess: Finally, $\mathcal{A}$ outputs a guess $\mu^{\prime} \in\{0,1\}$, and wins the game if $\mu=\mu^{\prime}$.

The advantage of $\mathcal{A}$ is defined as $\operatorname{Adv}_{R I B E, \mathcal{A}}^{I N D-C P A}\left(1^{\lambda}\right)=\left|\operatorname{Pr}\left[\mu=\mu^{\prime}\right]-\frac{1}{2}\right|$ where the probability is taken over all the randomness of the experiment. An RIBE scheme is fully secure under chosen plaintext attacks if for all PPT adversary $\mathcal{A}$, the advantage of $\mathcal{A}$ in the above experiment is negligible in the security parameter $\lambda$.

We can also define the selective revocation list security that is weaker than the previous full security of RIBE. In the selective revocation list model, an adversary should submits a challenge identity $I D^{*}$, challenge time $T^{*}$, and a revocation identity set $R^{*}$ at the time $T^{*}$ before the adversary receives public parameters. This model was introduced by Boldyreva et al. [4] to prove the security of their revocable ABE scheme.

Definition 2.4 (Selective Revocation List Security). The selective revocation list security of RIBE under chosen plaintext attacks is similar to the full security except that the adversary $\mathcal{A}$ submits a challenge identity $I D^{*}$, challenge time $T^{*}$, and a revoked identity set $R^{*}$ on the time $T^{*}$ before receiving public parameters. The advantage of $\mathcal{A}$ is defined as $\operatorname{Adv}_{R I B E, \mathcal{A}}^{S R L-I N A}\left(1^{\lambda}\right)=\left|\operatorname{Pr}\left[\mu=\mu^{\prime}\right]-\frac{1}{2}\right|$ where the probability is taken over all the randomness of the experiment. An RIBE scheme is secure in the selective revocation list model under chosen plaintext attacks if for all PPT adversary $\mathcal{A}$, the advantage of $\mathcal{A}$ in the above experiment is negligible in the security parameter $\lambda$.

\section{Revocable IBE with Selective Security}

In this section, we propose an RIBE scheme in prime-order bilinear groups and prove its security in the selective revocation list model under the standard assumption.

\subsection{Bilinear Groups of Prime Order}

Let $\mathbb{G}$ and $\mathbb{G}_{T}$ be two multiplicative cyclic groups of same prime order $p$ and $g$ be a generator of $\mathbb{G}$. The bilinear map $e: \mathbb{G} \times \mathbb{G} \rightarrow \mathbb{G}_{T}$ has the following properties:

1. Bilinearity: $\forall u, v \in \mathbb{G}$ and $\forall a, b \in \mathbb{Z}_{p}, e\left(u^{a}, v^{b}\right)=e(u, v)^{a b}$.

2. Non-degeneracy: $\exists g$ such that $e(g, g)$ has order $p$, that is, $e(g, g)$ is a generator of $\mathbb{G}_{T}$.

We say that $\mathbb{G}$ is a bilinear group if the group operations in $\mathbb{G}$ and $\mathbb{G}_{T}$ as well as the bilinear map $e$ are all efficiently computable. Furthermore, we assume that the description of $\mathbb{G}$ and $\mathbb{G}_{T}$ includes generators of $\mathbb{G}$ and $\mathbb{G}_{T}$ respectively. 


\subsection{Complexity Assumptions}

Assumption 1 (Decisional Bilinear Diffie-Hellman, DBDH). Let $\left(p, \mathbb{G}, \mathbb{G}_{T}, e\right)$ be a description of the bilinear group of prime order $p$. Let $g$ be generators of subgroups $\mathbb{G}$. The DBDH assumption is that if the challenge tuple

$$
D=\left(\left(p, \mathbb{G}, \mathbb{G}_{T}, e\right), g, g^{a}, g^{b}, g^{c}\right) \text { and } Z,
$$

are given, no PPT algorithm $\mathcal{A}$ can distinguish $Z=Z_{0}=e(g, g)^{a b c}$ from $Z=Z_{1}=e(g, g)^{d}$ with more than a negligible advantage. The advantage of $\mathcal{A}$ is defined as $\mathbf{A d} \mathbf{v}_{\mathcal{A}}^{D B D H}\left(1^{\lambda}\right)=\mid \operatorname{Pr}\left[\mathcal{A}\left(D, Z_{0}\right)=0\right]-\operatorname{Pr}\left[\mathcal{A}\left(D, Z_{1}\right)=\right.$ $0] \mid$ where the probability is taken over random choices of $a, b, c, d \in \mathbb{Z}_{p}$.

\subsection{Construction}

Let $\Delta_{i, I}$ be a Lagrange coefficient which is defined as $\Delta_{i, I}(x)=\prod_{j \in I, j \neq i} \frac{x-j}{i-j}$ for an index $i \in \mathbb{Z}_{p}$ and a set of indexes $I$ in $\mathbb{Z}_{p}$. Our RIBE scheme is described as follows:

RIBE.Setup $\left(1^{\lambda}, N_{\max }\right)$ : This algorithm takes as input a security parameter $1^{\lambda}$ and the maximum number $N_{\max }$ of users.

1. It first generates bilinear groups $\mathbb{G}, \mathbb{G}_{T}$ of prime order $p$ of bit size $\Theta(\lambda)$. Let $g$ be a random generator of $\mathbb{G}$. It selects a random exponent $\alpha \in \mathbb{Z}_{p}$ and random elements $u_{1}, h_{1}, u_{2}, h_{2} \in \mathbb{G}$. It initializes a user list $U L$ that contains a tuple $(I D, u)$, and also initializes a function list $F L$ that contains a tuple $\left(G L, f_{G L}(x)\right)$ for a group label $G L$.

2. It obtains $\mathcal{B} \mathcal{T}$ by running $\operatorname{SD.Setup}\left(N_{\max }\right)$. Let $\mathcal{S}$ be the collection of all subsets $S_{i, j}$ of $\mathcal{B T}$. For each $S_{i, j} \in \mathcal{S}$, it performs the following: It sets $G L=L_{i} \| d_{j}$ by obtaining $\left(L_{i}, L_{j}\right)=\operatorname{Label}\left(S_{i, j}\right)$ and $d_{j}=\operatorname{Depth}\left(S_{j}\right)$. If $(G L, *) \notin F L$, then it sets a random polynomial $f_{G L}(x)=a_{G L} x+\alpha$ by selecting a random $a_{G L} \in \mathbb{Z}_{p}$ and saves $\left(G L, f_{G L}(x)\right)$ to $F L$.

3. It outputs a master key $M K=(\alpha, F L)$, an empty revocation list $R L$, a state $S T=(\mathcal{B T}, U L)$, and public parameters $P P=\left(\left(p, \mathbb{G}, \mathbb{G}_{T}, e\right), g, u_{1}, h_{1}, u_{2}, h_{2}, \Omega=e(g, g)^{\alpha}\right)$.

RIBE.GenKey $(I D, M K, S T, P P)$ : This algorithm takes as input an identity $I D \in \mathcal{I}$, the master key $M K$, the state $S T=(\mathcal{B T}, U L)$, and public parameters $P P$.

1. It assigns the identity $I D$ to a leaf node $v_{u}$ in $\mathcal{B T}$ that is not yet assigned where $u \in \mathcal{N}$ is an index that is assigned to $I D$. It saves $(I D, u)$ to $U L$. Next, it obtains $P V_{u}=\left\{S_{i, j}\right\}$ by running $\operatorname{SD.Assign}(\mathcal{B T}, u)$.

2. For each $S_{i, j} \in P V_{u}$, it performs the following steps: It sets $G L=L_{i} \| d_{j}$ by obtaining $\left(L_{i}, L_{j}\right)=$ $\operatorname{Label}\left(S_{i, j}\right)$ and $d_{j}=\operatorname{Depth}\left(S_{j}\right)$, and then retrieves $\left(G L, f_{G L}(x)\right)$ from $F L$. Next, it selects a random exponent $r_{1} \in \mathbb{Z}_{p}$ and creates a personalized private key as

$$
P S K_{I D, S_{i, j}}=\left(K_{0}=g^{f_{G L}\left(L_{j}\right)}\left(u_{1}^{I D} h_{1}\right)^{r_{1}}, K_{1}=g^{-r_{1}}\right) .
$$

3. Finally, it outputs the updated state $S T$ and a private key $S K_{I D}=\left(P V_{u},\left\{P S K_{I D, S_{i, j}}\right\}_{S_{i, j} \in P V_{u}}\right)$.

RIBE.UpdateKey $(T, R L, M K, S T, P P)$ : This algorithm takes as input time $T$, the revocation list $R L$, the master key $M K$, the state $S T=(\mathcal{B T}, U L)$, and public parameters $P P$. 
1. It first defines the revoked set $R$ of user identities on the time $T$ from $R L$. That is, if there exists $\left(I D^{\prime}, T^{\prime}\right)$ such that $\left(I D^{\prime}, T^{\prime}\right) \in R L$ for any $T^{\prime} \leq T$, then $I D^{\prime} \in R$. It also defines the revoked index set $R I \subseteq \mathcal{N}$ of the revoked identity set $R$ by using $U L$. Next, it obtains $C V_{R I}=\left\{S_{i, j}\right\}$ by running $\operatorname{SD.Cover}(\mathcal{B T}, R I)$.

2. For each $S_{i, j} \in C V_{R}$, it performs the following steps: It sets $G L=L_{i} \| d_{j}$ by obtaining $\left(L_{i}, L_{j}\right)=$ $\operatorname{Label}\left(S_{i, j}\right)$ and $d_{j}=\operatorname{Depth}\left(S_{j}\right)$, and then retrieves $\left(G L, f_{G L}(x)\right)$ from $F L$. Next, it selects a random exponent $r_{2} \in \mathbb{Z}_{p}$ and creates a time-constrained update key as

$$
T U K_{T, S_{i, j}}=\left(U_{0}=g^{f_{G L}\left(L_{j}\right)}\left(u_{2}^{T} h_{2}\right)^{r_{2}}, U_{1}=g^{-r_{2}}\right) .
$$

3. Finally, it outputs the updated state $S T$ and an update key $U K_{T, R}=\left(C V_{R I},\left\{T U K_{T, S_{i, j}}\right\}_{S_{i, j} \in C V_{R I}}\right)$.

RIBE.DeriveKey $\left(S K_{I D}, U K_{T, R}, P P\right)$ : This algorithm takes as input a private key $S K_{I D}=\left(P V_{u},\left\{P S K_{I D, S_{i, j}}\right\}\right)$, an update key $U K_{T, R}=\left(C V_{R I},\left\{T U K_{T, S_{i, j}}\right\}\right)$, and the public parameters $P P$.

1. If $I D \notin R$, then it obtains $\left(S_{i, j}, S_{i^{\prime}, j^{\prime}}\right)$ by running $\mathbf{S D . M a t c h}\left(C V_{R I}, P V_{u}\right)$ such that $S_{i, j} \in C V_{R}$, $S_{i^{\prime}, j^{\prime}} \in P V_{u}$, and $\left(L_{i}=L_{i^{\prime}}\right) \wedge\left(d_{j}=d_{j^{\prime}}\right) \wedge\left(L_{j} \neq L_{j^{\prime}}\right)$ where $\left(L_{i}, L_{j}\right)=\operatorname{Label}\left(S_{i, j}\right),\left(L_{i^{\prime}}, L_{j}\right)=$ $\operatorname{Label}\left(S_{i^{\prime}, j^{\prime}}\right), d_{j}=\operatorname{Depth}\left(S_{j}\right)$, and $d_{j^{\prime}}=\operatorname{Depth}\left(S_{j^{\prime}}\right)$. Otherwise, it outputs $\perp$.

2. It retrieves $T U K_{T, S_{i, j}}=\left(U_{0}, U_{1}\right)$ from $U K_{T, R}$ and $P S K_{I D, S_{i^{\prime}, j^{\prime}}}=\left(K_{0}, K_{1}\right)$ from $S K_{I D}$. Note that $T U K_{T, S_{i, j}}$ and $P S K_{I D, S_{i^{\prime}, j^{\prime}}^{\prime}}$ share the same $f_{G L}(x)$ for $G L=L_{i} \| d_{j}$ since $\left(L_{i}=L_{i^{\prime}}\right) \wedge\left(d_{j}=d_{j^{\prime}}\right)$. Next, it sets $I=\left\{L_{j}, L_{j^{\prime}}\right\}$ and calculates two Lagrange coefficients $\Delta_{L_{j}, I}(0)$ and $\Delta_{L_{j^{\prime}}, I}(0)$ by using the fact $L_{j} \neq L_{j^{\prime}}$. It chooses random exponents $r_{1}^{\prime}, r_{2}^{\prime} \in \mathbb{Z}_{p}$ and creates decryption key components as

$$
\begin{aligned}
& D_{0}=\left(K_{0}\right)^{\Delta_{L^{\prime}, I}(0)}\left(U_{0}\right)^{\Delta_{L_{j}, I}(0)} \cdot\left(u_{1}^{I D} h_{1}\right)^{r_{1}^{\prime}}\left(u_{2}^{T} h_{2}\right)^{r_{2}^{\prime}}, \\
& D_{1}=\left(K_{1}\right)^{\Delta_{L_{j^{\prime}}, I}(0)} \cdot g^{-r_{1}^{\prime}}, D_{2}=\left(U_{1}\right)^{\Delta_{L_{j}, I}(0)} \cdot g^{-r_{2}^{\prime}} .
\end{aligned}
$$

3. Finally, it outputs a decryption key $D K_{I D, T}=\left(D_{0}, D_{1}, D_{2}\right)$. Note that the components are formed as $D_{0}=g^{\alpha}\left(u_{1}^{I D} h_{1}\right)^{r_{1}^{\prime \prime}}\left(u_{2}^{T} h_{2}\right)^{r_{2}^{\prime \prime}}, D_{1}=g^{-r_{1}^{\prime \prime}}, D_{2}=g^{-r_{2}^{\prime \prime}}$ since $f_{G L}(0)=\alpha$ where $r_{1}^{\prime \prime}=r_{1} \Delta_{L_{j^{\prime}}, I}(0)+r_{1}^{\prime}$ and $r_{2}^{\prime \prime}=r_{2} \Delta_{L_{j}, I}(0)+r_{2}^{\prime}$.

RIBE.Encrypt $(I D, T, M, P P)$ : This algorithm takes as input an identity $I D$, time $T$, a message $M$, and the public parameters $P P$. It first chooses a random exponent $s \in \mathbb{Z}_{p}$ and outputs a ciphertext by implicitly including $I D$ and $T$ as

$$
C T_{I D, T}=\left(C=\Omega^{s} \cdot M, C_{0}=g^{s}, C_{1}=\left(u_{1}^{I D} h_{1}\right)^{s}, C_{2}=\left(u_{2}^{T} h_{2}\right)^{s}\right) .
$$

RIBE.Decrypt $\left(C T_{I D, T}, D K_{I D^{\prime}, T^{\prime}}, P P\right)$ : This algorithm takes as input a ciphertext $C T_{I D, T}=\left(C, C_{0}, C_{1}, C_{2}\right)$, a decryption key $D K_{I D^{\prime}, T^{\prime}}=\left(D_{0}, D_{1}, D_{2}\right)$, and the public parameters $P P$. If $\left(I D=I D^{\prime}\right) \wedge\left(T=T^{\prime}\right)$, then it outputs the encrypted message $M$ as $M=C \cdot\left(\prod_{i=0}^{2} e\left(C_{i}, D_{i}\right)\right)^{-1}$. Otherwise, it outputs $\perp$.

RIBE.Revoke $(I D, T, R L, S T)$ : This algorithm takes as input an identity $I D$, revocation time $T$, the revocation list $R L$, and the state $S T=(\mathcal{B T}, U L, F L)$. If $(I D, *) \notin U L$, then it outputs $\perp$ since the private key of $I D$ was not generated. Otherwise, it adds $(I D, T)$ to $R L$. It outputs the updated revocation list $R L$.

Remark 2. As noted in Remark 1, we can easily handle the case $r=0$ by using a dummy revoked user. 


\subsection{Correctness}

Let $S K_{I D}$ be a private key for an identity $I D$, and $U K_{T, R}$ be an update key for time $T$ and a revoked identity set $R$. If $I D \notin R$, then two subsets $\left(S_{i, j}, S_{i^{\prime}, j^{\prime}}\right)$ such that $S_{i, j} \in C V_{R I}, S_{i^{\prime}, j^{\prime}} \in P V_{u}$, and $\left(L_{i}=L_{i^{\prime}}\right) \wedge\left(d_{j}=d_{j^{\prime}}\right) \wedge\left(L_{j} \neq\right.$ $L_{j^{\prime}}$ ) can be obtained from the correctness of the SD scheme. A decryption key for $I D$ and $T$ can be derived from $P S K_{I D, S_{i^{\prime}, j^{\prime}}}=\left(K_{0}, K_{1}\right)$ and $T U K_{T, S_{i, j}}=\left(U_{0}, U_{1}\right)$ as

$$
\begin{aligned}
& D_{0}=\left(K_{0}\right)^{\Delta_{j^{\prime}, I}(0)}\left(U_{0}\right)^{\Delta_{L_{j}, I}(0)}\left(u_{1}^{I D} h_{1}\right)^{r_{1}^{\prime}}\left(u_{2}^{T} h_{2}\right)^{r_{2}^{\prime}}
\end{aligned}
$$

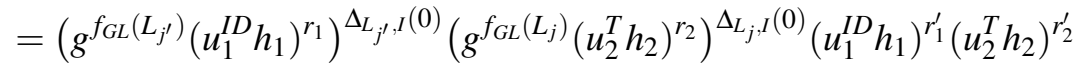

$$
\begin{aligned}
& =g^{f_{G L}(0)}\left(u_{1}^{I D} h_{1}\right)^{r_{1} \Delta_{L^{\prime}}, I}(0)+r_{1}^{\prime}\left(u_{2}^{T} h_{2}\right)^{r_{2} \Delta_{L_{j}, I}(0)+r_{2}^{\prime}}=g^{\alpha}\left(u_{1}^{I D} h_{1}\right)^{r_{1}^{\prime \prime}}\left(u_{2}^{T} h_{2}\right)^{r_{2}^{\prime \prime}} \text {, }
\end{aligned}
$$

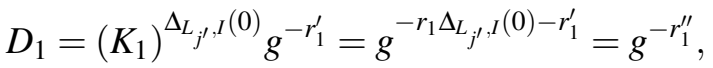

$$
\begin{aligned}
& D_{2}=\left(U_{1}\right)^{\Delta_{L_{j}, I}(0)} g^{-r_{2}^{\prime}}=g^{-r_{2} \Delta_{L_{j}, I}(0)-r_{2}^{\prime}}=g^{-r_{2}^{\prime \prime}}
\end{aligned}
$$

since $f_{G L}(0)=f_{G L}\left(L_{j^{\prime}}\right) \Delta_{L_{j^{\prime}}, I}(0)+f_{G L}\left(L_{j}\right) \Delta_{L_{j}, I}(0)=\left(a_{G L} L_{j^{\prime}}+\alpha\right) \frac{-L_{j}}{L_{j^{\prime}}-L_{j}}+\left(a_{G L} L_{j}+\alpha\right) \frac{-L_{j^{\prime}}}{L_{j}-L_{j^{\prime}}}=\alpha$ and $L_{j} \neq$ $L_{j^{\prime}}$ where $r_{1}^{\prime \prime}=r_{1} \Delta_{L_{j^{\prime}}, I}(0)+r_{1}^{\prime}$ and $r_{2}^{\prime \prime}=r_{2} \Delta_{L_{j}, I}(0)+r_{2}^{\prime}$. If $I D \in R$, then there are no subsets $\left(S_{i, j}, S_{i^{\prime}, j^{\prime}}\right)$ such that $S_{i, j} \in C V_{R I}, S_{i^{\prime}, j^{\prime}} \in P V_{u}$, and $\left(L_{i}=L_{i^{\prime}}\right) \wedge\left(d_{j}=d_{j^{\prime}}\right) \wedge\left(L_{j} \neq L_{j^{\prime}}\right)$ from the correctness of the SD scheme.

Let $C T_{I D, T}$ be a ciphertext for an identity $I D$ and time $T$, and $D K_{I D^{\prime}, T^{\prime}}$ be a decryption key for an identity $I D^{\prime}$ and time $T^{\prime}$. If $\left(I D=I D^{\prime}\right) \wedge\left(T=T^{\prime}\right)$, then the decryption algorithm correctly computes a session key by the following equation as

$$
\begin{aligned}
\prod_{i=0}^{2} e\left(C_{i}, D_{i}\right) & =e\left(g^{s}, g^{\alpha}\left(u_{1}^{I D} h_{1}\right)^{r_{1}^{\prime \prime}}\left(u_{2}^{T} h_{2}\right)^{r_{2}^{\prime \prime}}\right) \cdot e\left(\left(u_{1}^{I D} h_{1}\right)^{s}, g^{-r_{1}^{\prime \prime}}\right) \cdot e\left(\left(u_{2}^{T} h_{2}\right)^{s}, g^{-r_{2}^{\prime \prime}}\right) \\
& =e(g, g)^{\alpha s}=\Omega^{s} .
\end{aligned}
$$

\subsection{Security Analysis}

To prove the security of our RIBE scheme in the selective revocation list model, we use the partitioning method that was widely used for the security proof of other IBE schemes [5,6, 42]. In the security proof of IBE that uses the partitioning method, a simulator divides the identity space into two partitions where the simulator can generate a private key for an identity $I D \neq I D^{*}$ but he cannot generate a private key for a challenge identity $I D^{*}$. However, the direct use of the partitioning method does not work in RIBE since an adversary can request a private key for a challenge identity $I D^{*}$ and an update key for challenge time $T^{*}$ that were not allowed in the security model of IBE. That is, the simulator that uses the partitioning method of IBE cannot handle the private key for $I D^{*}$ and the update key for $T^{*}$.

To overcome this difficulty of using the partitioning method, we use the fact that a random polynomial $f(x)$ of degree one such that $f(0)=\alpha$ can be determined by one fixed point $(0, \alpha)$ and another random point $(\hat{x}, \hat{y})$. That is, if the adversary requests a private key for $I D^{*}$ or an update key for $T^{*}$, then the simulator directly uses the values $\hat{y}$ of the random point $(\hat{x}, \hat{y})$ by implicitly defining $f(\hat{x})=\hat{y}$ instead of using the Lagrange interpolation method to calculate $f\left(x^{\prime}\right)$ for some $x^{\prime}$ since the simulator cannot obtain an element for $f(0)=\alpha$ by using the partitioning method. To generate a private key for $I D^{*}$, the simulator assigns a random leaf node $v_{u^{*}}$ to the identity $I D^{*}$ and creates each personalized private key for a subset $S_{i, j}$ in $P V_{u^{*}}$ by using a random point $(\hat{x}, \hat{y})$ that implicitly defines a random polynomial $f_{G L}(x)$ for the group label $G L$. To generate an update key for $T^{*}$, the simulator obtains $C V_{R I^{*}}$ from the given revocation identity set $R^{*}$ and creates each time-constrained update key for a subset $S_{i, j}$ in $C V_{R I^{*}}$ by using a random point $(\hat{x}, \hat{y})$ 
that implicitly defines a random polynomial $f_{G L}(x)$ for the group label $G L$. Note that the simulation is only possible in the selective revocation list model since $R^{*}$ is needed to generate the update key for $T^{*}$.

However, the above proof idea is not enough to assure us the soundness of the proof. For the assurance, we should show that a subset $S_{i, j}$ of the private key query for $I D^{*}$ and a subset $S_{i, j^{\prime}}$ of the update key query for $T^{*}$ should be the same member label $L_{j}=L_{j^{\prime}}$ in a group label $G L$ if they belong to the same group label $G L$ to use the above simulation technique that uses a random point $(\hat{x}, \hat{y})$. At first, we have that each subset in the private set $P V_{u^{*}}$ for $I D^{*}$ belongs to different group labels since $P V_{u^{*}}$ is associated with a path, and each subset in the covering set $C V_{R I^{*}}$ for $T^{*}$ also belongs to different group labels since $C V_{R I^{*}}$ is a partition. If a subset $S_{i, j}$ of $P V_{u^{*}}$ and a subset $S_{i, j^{\prime}}$ of $C V_{R I^{*}}$ belong to the same group label $G L$, then $v_{j}=v_{j^{\prime}}$ by the correctness of the SD scheme since $I D^{*} \in R^{*}$. Thus, two subsets $S_{i, j}$ and $S_{i, j^{\prime}}$ should be the same member label $L_{j}=L_{j^{\prime}}$ in the group label $G L$.

Theorem 3.1. The above RIBE scheme is secure in the selective revocation list model under chosen plaintext attacks if the DBDH assumption holds. That is, for any PPT adversary $\mathcal{A}$, we have that $\boldsymbol{A d} v_{R I B E, \mathcal{A}}^{S R L-C P A}\left(1^{\lambda}\right) \leq$ $\boldsymbol{A d} \boldsymbol{v}_{\mathcal{B}}^{D B D H}\left(1^{\lambda}\right)$.

Proof. Suppose there exists an adversary $\mathcal{A}$ that attacks the above RIBE scheme with a non-negligible advantage. A simulator $\mathcal{B}$ that solves the DBDH assumption using $\mathcal{A}$ is given: a challenge tuple $D=$ $\left(\left(p, \mathbb{G}, \mathbb{G}_{T}, e\right), g, g^{a}, g^{b}, g^{c}\right)$ and $Z$ where $Z=Z_{0}=e(g, g)^{a b c}$ or $Z=Z_{1} \in_{R} \mathbb{G}_{T}$. Then $\mathcal{B}$ that interacts with $\mathcal{A}$ is described as follows:

Init: $\mathcal{A}$ initially submits a challenge identity $I D^{*}$, a challenge time $T^{*}$, and a revoked identity set $R^{*}$ on the time $T^{*}$.

Setup: $\mathcal{B}$ implicitly sets $\alpha=a b$ and proceeds as follows:

1. It first obtains $\mathcal{B} \mathcal{T}$ by running $\operatorname{SD.Setup}\left(N_{\max }\right)$. It initializes $U L$ and $F L$ as an empty one respectively. It assigns $I D^{*}$ to a random index $u^{*}$ and saves $\left(I D^{*}, u^{*}\right)$ to $U L$. For each $I D \in R^{*} \backslash\left\{I D^{*}\right\}$, it assigns $I D$ to a random index $u$ such that $(*, u) \notin U L$ and saves $(I D, u)$ to $U L$. From $R^{*}$, it also defines the revoked index set $R I^{*}$ by using $U L$.

2. It obtains $P V_{u^{*}}$ and $C V_{R I^{*}}$ by running $\operatorname{SD.Assign}\left(\mathcal{B} \mathcal{T}, u^{*}\right)$ and $\operatorname{SD.} \operatorname{Cover}\left(\mathcal{B} \mathcal{T}, R I^{*}\right)$ respectively. If $I D^{*} \in R^{*}$, then it sets FixedSubset $\left(I D^{*}, R^{*}\right)=P V_{u^{*}} \cup C V_{R I^{*}}$. Otherwise, it sets FixedSubset $\left(I D^{*}, R^{*}\right)=$ $C V_{R I^{*}}$. It sets the function list $F L$ as follows:

(a) For each $S_{i, j} \in$ FixedSubset $\left(I D^{*}, R^{*}\right)$, it selects a random value $\hat{y} \in \mathbb{Z}_{p}$ and saves $\left(G L=L_{i} \| d_{j},(\hat{x}=\right.$ $\left.\left.L_{j}, \hat{y}\right)\right)$ to $F L$ where $\left(L_{i}, L_{j}\right)=\operatorname{Label}\left(S_{i, j}\right)$ and $d_{j}=\operatorname{Depth}\left(S_{j}\right)$.

(b) For each $S_{i, j} \in \mathcal{S} \backslash$ FixedSubset $\left(I D^{*}, R^{*}\right)$, it selects random values $\hat{x}, \hat{y} \in \mathbb{Z}_{p}$ and saves $(G L=$ $\left.L_{i} \| d_{j},(\hat{x}, \hat{y})\right)$ to $F L$ if $\left(G L=L_{i} \| d_{j}, *\right) \notin F L$ where $\left(L_{i}, L_{j}\right)=\operatorname{Label}\left(S_{i, j}\right)$ and $d_{j}=\operatorname{Depth}\left(S_{j}\right)$.

Note that $f_{G L}(x)=a_{G L} x+\alpha$ for some $a_{G L} \in \mathbb{Z}_{p}$ is implicitly defined by two points $(0, \alpha)$ and $(\hat{x}, \hat{y})$ by using the Lagrange interpolation method. By defining the set FixedSubset $\left(I D^{*}, R^{*}\right)$ at the setup phase, we can handle a private key for $I D^{*}$ and an update key for $T^{*}$ without using the partitioning method. That is, we directly use the fixed point $\left(\hat{x}=L_{j}, \hat{y}\right)$ to generate the private key for $I D^{*}$ or the update key for $T^{*}$.

3. It sets $R L$ as an empty one and sets $S T=(\mathcal{B T}, U L)$. It selects random exponents $h_{0}^{\prime}, h_{1}^{\prime} \in \mathbb{Z}_{p}$ and publishes public parameters $P P$ as

$$
g, u_{1}=g^{a} g^{u_{1}^{\prime}}, h_{1}=\left(g^{a}\right)^{-I D^{*}} g^{h_{1}^{\prime}}, u_{2}=g^{a} g^{u_{2}^{\prime}}, h_{2}=\left(g^{a}\right)^{-T^{*}} g^{h_{2}^{\prime}}, \Omega=e\left(g^{a}, g^{b}\right) .
$$


Phase 1: $\mathcal{A}$ adaptively requests a polynomial number of private key, update key, and decryption key queries. If this is a private key query for an identity $I D$, then $\mathcal{B}$ proceeds as follows:

- Case $I D \neq I D^{*}$ : It performs the following steps:

1. It first selects a random exponent $r_{1}^{\prime} \in \mathbb{Z}_{p}$ and builds temporal private key components for the point $(0, \alpha)$ as $A_{0}=\left(g^{b}\right)^{-\left(u_{1}^{\prime} I D+h_{1}^{\prime}\right) /\left(I D-I D^{*}\right)}\left(u_{1}^{I D} h_{1}\right)^{r_{1}^{\prime}}, A_{1}=\left(g^{b}\right)^{1 /\left(I D-I D^{*}\right)} g^{-r_{1}^{\prime}}$.

2. If $(I D, *) \in U L$, then it loads $(I D, u)$ from $U L$. Otherwise, it selects a random index $u$ such that $\left(u \neq u^{*}\right) \wedge((*, u) \notin U L)$ and saves $(I D, u)$ to $U L$. Next, it obtains $P V_{u}=\left\{S_{i, j}\right\}$ by running $\operatorname{SD.Assign}(\mathcal{B T}, u)$.

3. For each $S_{i, j} \in P V_{u}$, it retrieves $\left(G L=L_{i} \| d_{j},(\hat{x}, \hat{y})\right)$ from $F L$ and performs the following:

- Case $S_{i, j} \in$ FixedSubset $\left(I D^{*}, R^{*}\right)$ : Recall that $\hat{x}=L_{j}$ from the setup phase. It selects a random exponent $r_{1} \in \mathbb{Z}_{p}$ and builds a personalized private key by implicitly setting $f_{G L}\left(L_{j}\right)=\hat{y}$ as

$$
P S K_{I D, S_{i, j}}=\left(K_{0}=g^{\hat{y}}\left(u_{1}^{I D} h_{1}\right)^{r_{1}}, K_{1}=g^{-r_{1}}\right) .
$$

- Case $S_{i, j} \notin$ FixedSubset $\left(I D^{*}, R^{*}\right)$ : Recall that $\hat{x} \neq L_{j}$ since $\hat{x}$ is a random value from the setup phase. It sets $I=\{0, \hat{x}\}$ and calculates two Lagrange coefficients $\Delta_{0, I}\left(L_{j}\right)$ and $\Delta_{\hat{x}, I}\left(L_{j}\right)$. Next, it selects a random exponents $r_{1}^{\prime \prime} \in \mathbb{Z}_{p}$ and builds a personalized private key as

$$
P S K_{I D, S_{i, j}}=\left(K_{0}=\left(A_{0}\right)^{\Delta_{0, I}\left(L_{j}\right)}\left(g^{\hat{y}}\right)^{\Delta_{\hat{x}, I}\left(L_{j}\right)} \cdot\left(u_{1}^{I D} h_{1}\right)^{r_{1}^{\prime \prime}}, K_{1}=\left(A_{1}\right)^{\Delta_{0, I}\left(L_{j}\right)} \cdot g^{-r_{1}^{\prime \prime}}\right) .
$$

4. Finally, it creates a private key $S K_{I D}=\left(P V_{u},\left\{P S K_{I D, S_{i, j}}\right\}_{S_{i, j} \in P V_{u}}\right)$.

- Case $I D=I D^{*}$ : In this case, we have $I D^{*} \in R^{*}$. It performs the following steps:

1. It loads $\left(I D^{*}, u^{*}\right)$ from $U L$ where $u^{*}$ is the pre-assigned index for $I D^{*}$. Next, it obtains $P V_{u^{*}}=$ $\left\{S_{i, j}\right\}$ by running $\mathbf{S D} . \operatorname{Assign}\left(\mathcal{B T}, u^{*}\right)$.

2. For each $S_{i, j} \in P V_{u^{*}}$, it retrieves $\left(G L=L_{i} \| d_{j},(\hat{x}, \hat{y})\right)$ from $F L$ and performs the following steps: Recall that $\hat{x}=L_{j}$ from the setup phase. It selects a random exponent $r_{1} \in \mathbb{Z}_{p}$ and builds a personalized private key by implicitly setting $f_{G L}\left(L_{j}\right)=\hat{y}$ as

$$
P S K_{I D^{*}, S_{i, j}}=\left(K_{0}=g^{\hat{y}}\left(u_{1}^{I D} h_{1}\right)^{r_{1}}, K_{1}=g^{-r_{1}}\right) .
$$

3. Finally, it creates a private key $S K_{I D^{*}}=\left(P V_{u^{*}},\left\{P S K_{I D^{*}, S_{i, j}}\right\}_{S_{i, j} \in P V_{u^{*}}}\right)$.

If this is an update key query for time $T$, then $\mathcal{B}$ proceeds as follows:

- Case $T \neq T^{*}$ : It performs the following steps.

1. It first selects a random exponent $r_{2}^{\prime} \in \mathbb{Z}_{p}$ and builds temporal update key components for the point $(0, \alpha)$ as $B_{0}=\left(g^{b}\right)^{-\left(u_{2}^{\prime} T+h_{2}^{\prime}\right) /\left(T-T^{*}\right)}\left(u_{2}^{T} h_{2}\right)^{r_{2}^{\prime}}, B_{1}=\left(g^{b}\right)^{1 /\left(T-T^{*}\right)} g^{-r_{2}^{\prime}}$.

2. It defines the revoked identity set $R$ on the time $T$ and the revoked index set $R I$ of $R$. Next, it obtains $C V_{R I}=\left\{S_{i, j}\right\}$ by running $\mathbf{S D . C o v e r}(\mathcal{B} \mathcal{T}, R I)$.

3. For each $S_{i, j} \in C V_{R I}$, it retrieves $\left(G L=L_{i} \| d_{j},(\hat{x}, \hat{y})\right)$ from $F L$ and performs the following: 
- Case $S_{i, j} \in$ FixedSubset $\left(I D^{*}, R^{*}\right)$ : It selects a random exponent $r_{2} \in \mathbb{Z}_{p}$ and creates a timeconstrained update key by implicitly setting $f_{G L}\left(L_{j}\right)=\hat{y}$ as

$$
T U K_{T, S_{i, j}}=\left(U_{0}=g^{y_{j}}\left(u_{2}^{T} h_{2}\right)^{r_{2}}, U_{1}=g^{-r_{2}}\right) .
$$

- Case $S_{i, j} \in$ FixedSubset $\left(I D^{*}, R^{*}\right)$ : It sets $I=\{0, \hat{x}\}$ and calculates two Lagrange coefficients $\Delta_{0, I}\left(L_{j}\right)$ and $\Delta_{\hat{z}, I}\left(L_{j}\right)$. Next, it selects a random exponent $r_{2}^{\prime \prime} \in \mathbb{Z}_{p}$ and builds a time-constrained update key as

$$
T U K_{T, S_{i, j}}=\left(U_{0}=\left(B_{0}\right)^{\Delta_{0, I}\left(L_{j}\right)}\left(g^{\hat{y}}\right)^{\Delta_{\hat{x}, I}\left(L_{j}\right)}\left(u_{2}^{T} h_{2}\right)^{r_{2}^{\prime \prime}}, U_{1}=\left(B_{1}\right)^{\Delta_{0, I}\left(L_{j}\right)} g^{-r_{2}^{\prime \prime}}\right) .
$$

4. Finally, it creates an update key $U K_{T, R}=\left(C V_{R I},\left\{T U K_{T, S_{i, j}}\right\}_{S_{i, j} \in C V_{R I}}\right)$.

- Case $T=T^{*}$ : In this case, we have $R=R^{*}$. It performs the following steps:

1. It first defines the revoked identity set $R$ on the time $T$ and the revoked index set $R I$ of $R$. Next, it obtains $C V_{R I^{*}}=\left\{S_{i, j}\right\}$ by running $\operatorname{SD.Cover}\left(\mathcal{B} \mathcal{T}, R I^{*}\right)$.

2. For each $S_{i, j} \in C V_{R I^{*}}$, it performs the following steps: It sets $G L=L_{i} \| d_{j}$ and retrieves $(G L,(\hat{x}, \hat{y}))$ from $F L$. Next, it selects a random exponent $r_{2} \in \mathbb{Z}_{p}$ and builds a time-constrained update key by implicitly setting $f_{G L}\left(L_{j}\right)=\hat{y}$ as

$$
T U K_{T^{*}, S_{i, j}}=\left(U_{0}=g^{\hat{y}}\left(u_{2}^{T^{*}} h_{2}\right)^{r_{2}}, U_{1}=g^{-r_{2}}\right) .
$$

3. Finally, it creates an update key $\left.U K_{T^{*}, R^{*}}=\left(C V_{R I^{*}},\left\{T U K_{T^{*}, S_{i, j}}\right)\right\}_{S_{i, j} \in C V_{R I^{*}}}\right)$.

If this is a decryption key query for an identity $I D$ and a time $T$, then $\mathcal{B}$ proceeds as follows:

- Case $I D \neq I D^{*}$ : If $(I D, *) \notin U L$, then it selects a random index $u$ such that $(*, u) \notin U L$ and saves $(I D, u)$ to $U L$. It selects random exponents $r_{1}^{\prime}, r_{2} \in \mathbb{Z}_{p}$ and creates a decryption key $D K_{I D, T}$ by implicitly setting $r_{1}=-b /\left(I D-I D^{*}\right)+r_{1}^{\prime}$ as

$$
D_{0}=\left(g^{b}\right)^{-\left(u_{1}^{\prime} I D+h_{1}^{\prime}\right) /\left(I D-I D^{*}\right)}\left(u_{1}^{I D} h_{1}\right)^{r_{1}^{\prime}}\left(u_{2}^{T} h_{2}\right)^{r_{2}}, D_{1}=\left(g^{b}\right)^{1 /\left(I D-I D^{*}\right)} g^{-r_{1}^{\prime}}, D_{2}=g^{-r_{2}} .
$$

- Case $I D=I D^{*}$ : In this case, we have $T \neq T^{*}$ from the restriction of Definition 2.4. It selects random exponents $r_{1}, r_{2}^{\prime} \in \mathbb{Z}_{p}$ and creates a decryption key $D K_{I D, T}$ implicitly setting $r_{2}=-b /\left(T-T^{*}\right)+r_{2}^{\prime}$ as

$$
D_{0}=\left(u_{1}^{I D} h_{1}\right)^{r_{1}}\left(g^{b}\right)^{-\left(u_{2}^{\prime} T+h_{2}^{\prime}\right) /\left(T-T^{*}\right)}\left(u_{2}^{T} h_{2}\right)^{r_{2}^{\prime}}, D_{1}=g^{-r_{1}}, D_{2}=\left(g^{b}\right)^{1 /\left(T-T^{*}\right)} g^{-r_{2}^{\prime}} .
$$

If this is a revocation query for an identity $I D$ and time $T$, then $\mathcal{B}$ updates $R L$ by running RIBE.Revoke $(I D$, $T, R L, S T)$.

Challenge: $\mathcal{A}$ submits two challenge messages $M_{0}^{*}, M_{1}^{*} . \mathcal{B}$ chooses a random bit $\mu \in\{0,1\}$ and creates the challenge ciphertext $C T^{*}$ by implicitly setting $s=c$ as

$$
C=Z \cdot M_{\mu}^{*}, C_{0}=g^{c}, C_{1}=\left(g^{c}\right)^{u_{1}^{\prime} I D^{*}+h_{1}^{\prime}}, C_{2}=\left(g^{c}\right)^{u_{2}^{\prime} T^{*}+h_{2}^{\prime}} .
$$

Phase 2: Same as Phase 1. 
Guess: Finally, $\mathcal{A}$ outputs a guess $\mu^{\prime} \in\{0,1\} . \mathcal{B}$ outputs 0 if $\mu=\mu^{\prime}$ or 1 otherwise.

To finish the proof, we first show that the simulation is correct. The public parameters is correct since random exponents $u_{1}^{\prime}, h_{1}^{\prime}, u_{2}^{\prime}, h_{2}^{\prime} \in \mathbb{Z}_{p}$ are chosen. We show that the private keys are correct. In case of $I D \neq I D^{*}$, we have that a personalized private key for $S_{i, j}$ such that $S_{i, j} \notin \operatorname{FixedSubset}\left(I D^{*}, R^{*}\right)$ is correctly distributed from the setting $r_{1}=\left(-b /\left(I D-I D^{*}\right)+r_{1}^{\prime}\right) \Delta_{0, I}\left(L_{j}\right)+r_{1}^{\prime \prime}$ as

$$
\begin{aligned}
K_{0} & =g^{f_{G L}\left(L_{j}\right)}\left(u_{1}^{I D} h_{1}\right)^{r_{1}}=g^{\alpha \Delta_{0, I}\left(L_{j}\right)+\hat{y} \Delta_{\hat{,}, I}\left(L_{j}\right)}\left(u_{1}^{I D} h_{1}\right)^{\tilde{r}_{1} \Delta_{0, I}\left(L_{j}\right)+r_{1}^{\prime \prime}} \\
& =\left(g^{\alpha}\left(u_{1}^{I D} h_{1}\right)^{\tilde{r}_{1}}\right)^{\Delta_{0, I}\left(L_{j}\right)} g^{\hat{y} \Delta_{\hat{x}, I}\left(L_{j}\right)}\left(u_{1}^{I D} h_{1}\right)^{r_{1}^{\prime \prime}} \\
& =\left(g^{a b}\left(\left(g^{a}\right)^{I D-I D^{*}} g^{u_{1}^{\prime} I D+h_{1}^{\prime}}\right)^{-b /\left(I D-I D^{*}\right)+r_{1}^{\prime}}\right)^{\Delta_{0, I}\left(L_{j}\right)} g^{\hat{y} \Delta_{\hat{x}, I}\left(x_{j}\right)}\left(u_{1}^{I D} h_{1}\right)^{r_{1}^{\prime \prime}} \\
& =\left(\left(g^{b}\right)^{-\left(u_{1}^{\prime} I D+h_{1}^{\prime}\right) /\left(I D-I D^{*}\right)}\left(u_{1}^{I D} h_{1}\right)^{r_{1}^{\prime}}\right)^{\Delta_{0, I}\left(L_{j}\right)} g^{\hat{y} \Delta_{\hat{x}, I}\left(L_{j}\right)}\left(u_{1}^{I D} h_{1}\right)^{r_{1}^{\prime \prime}}, \\
K_{1} & =g^{-r_{1}}=g^{\left(b /\left(I D-I D^{*}\right)-r_{1}^{\prime}\right) \Delta_{0, I}\left(L_{j}\right)-r_{1}^{\prime \prime}}=\left(\left(g^{b}\right)^{1 /\left(I D-I D^{*}\right)} g^{-r_{1}^{\prime}}\right)^{\Delta_{0, I}\left(L_{j}\right)} g^{-r_{1}^{\prime \prime}} .
\end{aligned}
$$

In case of $I D=I D^{*}$, we have that a personalized private key for $S_{i, j}$ is correctly distributed from the setting $L_{j}=\hat{x}$ and $f_{G L}(\hat{x})=\hat{y}$ as

$$
K_{0}=g^{f_{G L}\left(L_{j}\right)}\left(u_{1}^{I D} h_{1}\right)^{r_{1}}=g^{\hat{y}}\left(u_{1}^{I D} h_{1}\right)^{r_{1}}, K_{1}=g^{-r_{1}} .
$$

Next, we show that the update keys are correct. In case of $T \neq T^{*}$, we have that a time-constrained update key for $S_{i, j}$ such that $S_{i, j} \notin$ FixedSubset $\left(I D^{*}, R^{*}\right)$ is correctly distributed from the setting $r_{2}=(-b /(T-$ $\left.\left.T^{*}\right)+r_{2}^{\prime}\right) \Delta_{0, I}\left(L_{j}\right)+r_{2}^{\prime \prime}$ as

$$
\begin{aligned}
U_{0} & =g^{f_{G L}\left(L_{j}\right)}\left(u_{2}^{T} h_{2}\right)^{r_{2}}=g^{\alpha \Delta_{0, I}\left(L_{j}\right)+\hat{y} \Delta_{\hat{x}, I}\left(L_{j}\right)}\left(u_{2}^{T} h_{2}\right)^{\tilde{r}_{2} \Delta_{0, I}\left(L_{j}\right)+r_{2}^{\prime \prime}} \\
& =\left(g^{\alpha}\left(u_{2}^{T} h_{2}\right)^{\tilde{r}_{2}}\right)^{\Delta_{0, I}\left(L_{j}\right)} g^{\hat{y} \Delta_{\hat{,}, I}\left(L_{j}\right)}\left(u_{2}^{T} h_{2}\right)^{r_{2}^{\prime \prime}} \\
& =\left(g^{a b}\left(\left(g^{a}\right)^{T-T^{*}} g^{u_{2}^{\prime} T+h_{2}^{\prime}}\right)^{-b /\left(T-T^{*}\right)+r_{2}^{\prime}}\right)^{\Delta_{0, I}\left(L_{j}\right)} g^{\hat{y} \Delta_{\hat{x}, I}\left(L_{j}\right)}\left(u_{2}^{T} h_{2}\right)^{r_{2}^{\prime \prime}} \\
& =\left(\left(g^{b}\right)^{-\left(u_{2}^{\prime} T+h_{2}^{\prime}\right) /\left(T-T^{*}\right)}\left(u_{2}^{T} h_{2}\right)^{r_{2}^{\prime}}\right)^{\Delta_{0, I}\left(L_{j}\right)} g^{\hat{y} \Delta_{\hat{x}, I}\left(L_{j}\right)}\left(u_{2}^{T} h_{2}\right)^{r_{2}^{\prime \prime}}, \\
U_{1} & =g^{-r_{2}}=g^{\left(b /\left(T-T^{*}\right)-r_{2}^{\prime}\right) \Delta_{0, I}\left(L_{j}\right)-r_{2}^{\prime \prime}}=\left(\left(g^{b}\right)^{1 /\left(T-T^{*}\right)} g^{-r_{2}^{\prime}}\right)^{\Delta_{0, I}\left(L_{j}\right)} g^{-r_{2}^{\prime \prime}} .
\end{aligned}
$$

In case of $T=T^{*}$, we have that a time-constrained update key for $S_{i, j}$ is correctly distributed from the setting $L_{j}=\hat{x}$ and $f_{G L}(\hat{x})=\hat{y}$ as

$$
U_{0}=g^{f_{G L}\left(L_{j}\right)}\left(u_{2}^{T} h_{2}\right)^{r_{2}}=g^{\hat{y}}\left(u_{2}^{T} h_{2}\right)^{r_{2}}, U_{1}=g^{-r_{2}} .
$$

We show that the decryption keys are correct. In case of $I D \neq I D^{*}$, the decryption key is correctly distributed by setting $r_{1}=-b /\left(I D-I D^{*}\right)+r_{1}^{\prime}$ as

$$
\begin{aligned}
D_{0} & =g^{\alpha}\left(u_{1}^{I D} h_{1}\right)^{r_{1}}\left(u_{2}^{T} h_{2}\right)^{r_{2}}=g^{a b}\left(\left(g^{a}\right)^{I D-I D^{*}} g^{u_{1}^{\prime} I D+h_{1}^{\prime}}\right)^{-b /\left(I D-I D^{*}\right)+r_{1}^{\prime}}\left(u_{2}^{T} h_{2}\right)^{r_{2}} \\
& =\left(g^{b}\right)^{-\left(u_{1}^{\prime} I D+h_{1}^{\prime}\right) /\left(I D-I D^{*}\right)}\left(u_{1}^{I D} h_{1}\right)^{r_{1}^{\prime}}\left(u_{2}^{T} h_{2}\right)^{r_{2}}, \\
D_{1} & =g^{-r_{1}}=g^{b /\left(I D-I D^{*}\right)-r_{1}^{\prime}}=\left(g^{b}\right)^{1 /\left(I D-I D^{*}\right)} g^{-r_{1}^{\prime}} .
\end{aligned}
$$

In case of $I D=I D^{*}$, the decryption key is correctly distributed by setting $r_{2}=-b /\left(T-T^{*}\right)+r_{2}^{\prime}$ as

$$
\begin{aligned}
D_{0} & =g^{\alpha}\left(u_{1}^{I D} h_{1}\right)^{r_{1}}\left(u_{2}^{T} h_{2}\right)^{r_{2}}=g^{a b}\left(u_{1}^{I D} h_{1}\right)^{r_{1}}\left(\left(g^{a}\right)^{T-T^{*}} g^{u_{2}^{\prime} T+h_{2}^{\prime}}\right)^{-b /\left(T-T^{*}\right)+r_{2}^{\prime}} \\
& =\left(u_{1}^{I D} h_{1}\right)^{r_{1}}\left(g^{b}\right)^{-\left(u_{2}^{\prime} T+h_{2}^{\prime}\right) /\left(T-T^{*}\right)}\left(u_{2}^{T} h_{2}\right)^{r_{2}^{\prime}}, \\
D_{2} & =g^{-r_{2}}=g^{b /\left(T-T^{*}\right)-r_{2}^{\prime}}=\left(g^{b}\right)^{1 /\left(T-T^{*}\right)} g^{-r_{2}^{\prime}} .
\end{aligned}
$$


Finally, we show that the challenge ciphertext is correct. If $Z=Z_{0}=e(g, g)^{a b c}$ is given, then the challenge ciphertext is correctly distributed as

$$
\begin{aligned}
& C=\Omega^{s}=e(g, g)^{\alpha s}=e(g, g)^{a b c}, C_{0}=g^{s}=g^{c}, \\
& C_{1}=\left(u_{1}^{I D^{*}} h_{1}\right)^{s}=\left(\left(g^{a}\right)^{I D^{*}-I D^{*}} g^{u_{1}^{\prime} I D^{*}+h_{1}^{\prime}}\right)^{s}=\left(g^{c}\right)^{u_{1}^{\prime} I D^{*}+h_{1}^{\prime}}, \\
& C_{2}=\left(u_{2}^{T^{*}} h_{2}\right)^{s}=\left(\left(g^{a}\right)^{T^{*}-T^{*}} g^{u_{2}^{\prime} T^{*}+h_{2}^{\prime}}\right)^{s}=\left(g^{c}\right)^{u_{2}^{\prime} T^{*}+h_{2}^{\prime}} .
\end{aligned}
$$

Otherwise, the component $C$ of the challenge ciphertext is independent of $\delta$ in the $\mathcal{A}$ 's view since $Z_{1}$ is a random element in $\mathbb{G}_{T}$.

Let $\eta$ be a random bit for $Z_{\eta}$. From the above simulation, we have $\operatorname{Pr}\left[\mu=\mu^{\prime} \mid \eta=0\right]=\frac{1}{2}+\mathbf{A d d} \mathbf{v}_{R I B E, \mathcal{A}}^{S R L-C P A}\left(1^{\lambda}\right)$ since the distribution of the simulation is correct, and we also have $\operatorname{Pr}\left[\mu=\mu^{\prime} \mid \eta=1\right]=\frac{1}{2}$ since $\mu$ is completely hidden to $\mathcal{A}$. Therefore we can obtain the following equation

$$
\begin{aligned}
\operatorname{Adv}_{\mathcal{B}}^{D B D H}\left(1^{\lambda}\right) & =\left|\operatorname{Pr}\left[\mathcal{B}\left(D, Z_{0}\right)=0\right]-\operatorname{Pr}\left[\mathcal{B}\left(D, Z_{1}\right)=0\right]\right|=\left|\operatorname{Pr}\left[\mu=\mu^{\prime} \mid \eta=0\right]\right|-\left|\operatorname{Pr}\left[\mu=\mu^{\prime} \mid \eta=1\right]\right| \\
& =\frac{1}{2}+\mathbf{A d v}_{R I B E, \mathcal{A}}^{S R L-I N A}\left(1^{\lambda}\right)-\frac{1}{2}=\mathbf{A d v}_{R I B E, \mathcal{A}}^{S R L-C N D-C P A}\left(1^{\lambda}\right) .
\end{aligned}
$$

This completes our proof.

\subsection{Discussions}

Efficiency. In our RIBE scheme that employs the SD scheme, the public parameters, a private key, an update key, and a ciphertext consist of $O(1), O\left(\log ^{2} N_{\max }\right), O(r)$, and $O(1)$ number of group elements respectively where $N_{\max }$ is the maximum number of users and $r$ is the number of revoked users in an update key. Compared with the previous RIBE scheme that employs the CS scheme that has $O\left(\log N_{\max }\right)$ number of group elements in a private key and $O\left(r \log \left(N_{\max } / r\right)\right)$ number of group elements in an update key, our RIBE scheme can reduce the number of group elements in an update key by increasing the number of group elements in a private key. If we use the LSD scheme of Halevy and Shamir [15] instead of the SD scheme, then we can reduce the number of group element in a private key to $O\left(\log ^{1.5} N_{\max }\right)$.

Removing Stored Polynomials. The setup algorithm of our RIBE scheme should maintain the function list $F L$ that stores a random polynomial $f(x)$ of degree one for each group label in a tree. If $N_{\max }$ is the maximum number of users in the system, then the maximum number of group labels is $O\left(N_{\max } \log N_{\max }\right)$ since a group label is defined by a node $v_{i}$ that is not a leaf node in the tree and a depth $d_{j}$ in the tree. A pseudo-random function $P R F$ can be used to remove $F L$. That is, the master key $M K$ consists of a random exponent $\alpha$ and a seed $z$ for PRF, and a random polynomial $f_{G L}(x)$ for a group label can be defined as $f_{G L}(x)=a_{G L} x+\alpha$ where $G L$ is a group label and $a_{G L}=P R F_{z}(G L)$. The security of this modified scheme also holds from the security of the pseudo-random function.

Supporting an Exponential Number of Users. Our RIBE scheme takes the maximum number of users $N_{\max }$ as an input and assigns each user to a leaf node of a binary tree with depth $\log N_{\max }$. To support an exponential number of users, a binary tree with depth $2 \lambda$ can be used where $\lambda$ is a security parameter and the bit size of an identity is $2 \lambda$. Additionally, a random function $f_{G L}(x)$ can be deterministically generated by using a pseudo-random function $P R F$ instead of keeping a function list $F L$. Furthermore, if a user is assigned to a leaf node of a tree such that the label $L$ of the leaf node is equal to the identity string $I D$, then the user list $U L$ is not needed.

Layered Subset Difference. Compared with the previous RIBE scheme that employs the CS scheme, our RIBE scheme that uses the SD scheme reduce the number of group elements in update keys from 
$O\left(r \log \left(N_{\max } / r\right)\right)$ to $O(r)$, but it increases the number of group elements in private keys from $O\left(\log N_{\max }\right)$ to $O\left(\log ^{2} N_{\max }\right)$. To reduce the size of private keys in RIBE, we can use the layered subset difference (LSD) scheme of Halevy and Shamir [15]. In the LSD scheme, the number of subsets in a private set $P V_{u}$ is $O\left(\log { }^{1.5} N_{\max }\right)$ and the number of subsets in a covering set $C V_{R}$ is still $O(r)$. Our RIBE scheme also can employ the LSD scheme since the LSD scheme is a special case of the SD scheme. The security proof of this RIBE scheme that uses the LSD scheme also holds.

Comparison with the RIBE scheme of Boldyreva et al. Compared with the previous RIBE schemes, our RIBE scheme and the RIBE scheme of Boldyreva et al. [4] have the similarity of using a random polynomial of degree one. However, the purpose of using a random polynomial is quite different between two schemes. That is, we use a random polynomial of degree one for single member revocation to integrate it with the SD scheme whereas they use a degree one polynomial for the FIBE scheme of Sahai and Waters [37].

Chosen-Ciphertext Security. The proposed RIBE scheme only provides the indistinguishability under chosen-plaintext attacks (IND-CPA). To provide the stronger indistinguishability under chosen-ciphertext attacks (IND-CCA) where an adversary can request decryption queries, we can use the general transformation of Canetti, Halevi, and Katz [9] since our RIBE scheme can be easily modified to support the HIBE scheme with 3-level of Boneh and Boyen [5]. That is, we can use the additional level of HIBE to provide the integrity of ciphertexts by using an one-time signature scheme. The proof of IND-CCA security easily follows since the decryption queries can be easily simulated by the private key delegation capability of the HIBE scheme.

Achieving Full Security. The security of our RIBE scheme is only proven in the selective revocation list model that is weaker than the well-known selective model since the revocation identity set $R^{*}$ should be additionally given. The previous RIBE schemes that employ the CS scheme were already proven in the full model by using a fully secure IBE scheme since the assigned key of a subset in the CS scheme is independent with each other [26, 39]. However, it is not easy to prove the full security of our RIBE scheme by using a fully secure IBE scheme and a partitioning method since the assigned key of a subset in the SD scheme is dependent of each member in a group label. In Section 4, we show that our RIBE scheme in compositeorder bilinear groups can be proven in the full model if we use the dual system encryption technique of Waters [25,43] instead of using the partitioning method.

\section{Revocable IBE with Full Security}

In this section, we propose an RIBE scheme in composite-order bilinear groups and prove its full security under static assumptions.

\subsection{Bilinear Groups of Composite Order}

Let $N=p_{1} p_{2} p_{3}$ where $p_{1}, p_{2}$, and $p_{3}$ are distinct prime numbers. Let $\mathbb{G}$ and $\mathbb{G}_{T}$ be two multiplicative cyclic groups of same composite order $N$ and $g$ be a generator of $\mathbb{G}$. The bilinear map $e: \mathbb{G} \times \mathbb{G} \rightarrow \mathbb{G}_{T}$ has the following properties:

1. Bilinearity: $\forall u, v \in \mathbb{G}$ and $\forall a, b \in \mathbb{Z}_{N}, e\left(u^{a}, v^{b}\right)=e(u, v)^{a b}$.

2. Non-degeneracy: $\exists g$ such that $e(g, g)$ has order $N$, that is, $e(g, g)$ is a generator of $\mathbb{G}_{T}$.

We say that $\mathbb{G}$ is a bilinear group if the group operations in $\mathbb{G}$ and $\mathbb{G}_{T}$ as well as the bilinear map $e$ are all efficiently computable. Furthermore, we assume that the description of $\mathbb{G}$ and $\mathbb{G}_{T}$ includes generators 
of $\mathbb{G}$ and $\mathbb{G}_{T}$ respectively. We use the notation $\mathbb{G}_{p_{i}}$ to denote the subgroups of order $p_{i}$ of $\mathbb{G}$ respectively. Similarly, we use the notation $\mathbb{G}_{T, p_{i}}$ to denote the subgroups of order $p_{i}$ of $\mathbb{G}_{T}$ respectively.

\subsection{Complexity Assumptions}

We present three static assumptions that were introduced by Lewko and Waters [25].

Assumption 2 (Subgroup Decision). Let $\left(N, \mathbb{G}, \mathbb{G}_{T}, e\right)$ be a description of the bilinear group of composite order $N=p_{1} p_{2} p_{3}$. Let $g_{p_{1}}, g_{p_{2}}, g_{p_{3}}$ be generators of subgroups $\mathbb{G}_{p_{1}}, \mathbb{G}_{p_{2}}, \mathbb{G}_{p_{3}}$ respectively. The Assumption is that if the challenge tuple

$$
D=\left(\left(N, \mathbb{G}, \mathbb{G}_{T}, e\right), g_{p_{1}}, g_{p_{3}}\right) \text { and } Z,
$$

are given, no PPT algorithm $\mathcal{A}$ can distinguish $Z=Z_{0}=X_{1}$ from $Z=Z_{1}=X_{1} R_{1}$ with more than a negligible advantage. The advantage of $\mathcal{A}$ is defined as $\operatorname{Adv}_{\mathcal{A}}^{S D}\left(1^{\lambda}\right)=\left|\operatorname{Pr}\left[\mathcal{A}\left(D, Z_{0}\right)=0\right]-\operatorname{Pr}\left[\mathcal{A}\left(D, Z_{1}\right)=0\right]\right|$ where the probability is taken over random choices of $X_{1} \in \mathbb{G}_{p_{1}}$ and $R_{1} \in \mathbb{G}_{p_{2}}$.

Assumption 3 (General Subgroup Decision). Let $\left(N, \mathbb{G}, \mathbb{G}_{T}, e\right)$ be a description of the bilinear group of composite order $N=p_{1} p_{2} p_{3}$. Let $g_{p_{1}}, g_{p_{2}}, g_{p_{3}}$ be generators of subgroups $\mathbb{G}_{p_{1}}, \mathbb{G}_{p_{2}}, \mathbb{G}_{p_{3}}$ respectively. The Assumption is that if the challenge tuple

$$
D=\left(\left(N, \mathbb{G}, \mathbb{G}_{T}, e\right), g_{p_{1}}, g_{p_{3}}, X_{1} R_{1}, R_{2} Y_{1}\right) \text { and } Z,
$$

are given, no PPT algorithm $\mathcal{A}$ can distinguish $Z=Z_{0}=X_{2} Y_{2}$ from $Z=Z_{1}=X_{2} R_{3} Y_{2}$ with more than a negligible advantage. The advantage of $\mathcal{B}$ is defined as $\operatorname{Adv}_{\mathcal{A}}^{G S D}\left(1^{\lambda}\right)=\mid \operatorname{Pr}\left[\mathcal{A}\left(D, Z_{0}\right)=0\right]-\operatorname{Pr}\left[\mathcal{A}\left(D, Z_{1}\right)=\right.$ $0] \mid$ where the probability is taken over random choices of $X_{1}, X_{2} \in \mathbb{G}_{p_{1}}, R_{1}, R_{2}, R_{3} \in \mathbb{G}_{p_{2}}$, and $Y_{1}, Y_{2} \in \mathbb{G}_{p_{3}}$.

Assumption 4 (Composite Diffie-Hellman). Let $\left(N, \mathbb{G}, \mathbb{G}_{T}, e\right)$ be a description of the bilinear group of composite order $N=p_{1} p_{2} p_{3}$. Let $g_{p_{1}}, g_{p_{2}}, g_{p_{3}}$ be generators of subgroups $\mathbb{G}_{p_{1}}, \mathbb{G}_{p_{2}}, \mathbb{G}_{p_{3}}$ respectively. The Assumption is that if the challenge tuple

$$
D=\left(\left(N, \mathbb{G}, \mathbb{G}_{T}, e\right), g_{p_{1}}, g_{p_{2}}, g_{p_{3}}, g_{p_{1}}^{a} R_{1}, g_{p_{1}}^{b} R_{2}\right) \text { and } Z,
$$

are given, no PPT algorithm $\mathcal{A}$ can distinguish $Z=Z_{0}=e\left(g_{p_{1}}, g_{p_{1}}\right)^{a b}$ from $Z=Z_{1}=e\left(g_{p_{1}}, g_{p_{1}}\right)^{c}$ with more than a negligible advantage. The advantage of $\mathcal{A}$ is defined as $\operatorname{Adv}_{\mathcal{A}}^{\operatorname{ComDH}}\left(1^{\lambda}\right)=\mid \operatorname{Pr}\left[\mathcal{A}\left(D, Z_{0}\right)=\right.$ $0]-\operatorname{Pr}\left[\mathcal{A}\left(D, Z_{1}\right)=0\right] \mid$ where the probability is taken over random choices of $a, b, c \in \mathbb{Z}_{N}$, and $R_{1}, R_{2} \in \mathbb{G}_{p_{2}}$.

\subsection{Construction}

Let $\Delta_{i, I}$ be a Lagrange coefficient which is defined as $\Delta_{i, I}(x)=\prod_{j \in I, j \neq i} \frac{x-j}{i-j}$ for an index $i \in \mathbb{Z}_{N}$ and a set of indexes $I$ in $\mathbb{Z}_{N}$. Our RIBE scheme is described as follows:

RIBE.Setup $\left(1^{\lambda}, N_{\max }\right)$ : This algorithm takes as input a security parameter $1^{\lambda}$ and the maximum number $N_{\max }$ of users.

1. It first generates a bilinear group $\mathbb{G}$ of composite order $N=p_{1} p_{2} p_{3}$ where $p_{1}, p_{2}$, and $p_{3}$ are random primes. Let $g_{1}$ be a random generator of $\mathbb{G}_{p_{1}}$. It selects a random exponent $\alpha \in \mathbb{Z}_{N}$ and random elements $u_{1}, h_{1}, u_{2}, h_{2} \in \mathbb{G}_{p_{1}}, Y \in \mathbb{G}_{p_{3}}$. It initializes a user list $U L$ that contains a tuple $(I D, u)$, and also initializes a function list $F L$ that contains a tuple $\left(G L, f_{G L}(x)\right)$ for a group label $G L$. 
2. It obtains $\mathcal{B} \mathcal{T}$ by running $\operatorname{SD.Setup}\left(N_{\max }\right)$. Let $\mathcal{S}$ be the collection of all subsets $S_{i, j}$ of $\mathcal{B T}$. For each $S_{i, j} \in \mathcal{S}$, it performs the following steps: It sets $G L=L_{i} \| d_{j}$ by obtaining $\left(L_{i}, L_{j}\right)=$ $\operatorname{Label}\left(S_{i, j}\right)$ and $d_{j}=\operatorname{Depth}\left(S_{j}\right)$. If $(G L, *) \notin F L$, then it sets a random polynomial $f_{G L}(x)=$ $a_{G L} x+\alpha$ by selecting a random $a_{G L} \in \mathbb{Z}_{N}$ and saves $\left(G L, f_{G L}(x)\right)$ to $F L$.

3. It outputs a master key $M K=(\alpha, F L)$, an empty revocation list $R L$, a state $S T=(\mathcal{B T}, U L)$, and public parameters $P P=\left(\left(N, \mathbb{G}, \mathbb{G}_{T}, e\right), g=g_{1}, Y, u_{1}, h_{1}, u_{2}, h_{2}, \Omega=e(g, g)^{\alpha}\right)$.

RIBE.GenKey $(I D, M K, S T, P P)$ : This algorithm takes as input an identity $I D \in \mathcal{I}$, the master key $M K$, the state $S T=(\mathcal{B T}, U L)$, and public parameters $P P$.

1. It first randomly assigns the identity $I D$ to a leaf node $v_{u}$ in $\mathcal{B} \mathcal{T}$ that is not yet assigned where $u \in \mathcal{N}$ is an index assigned to $I D$. It saves $(I D, u)$ to $U L$. Next, it obtains $P V_{u}=\left\{S_{i, j}\right\}$ by running $\operatorname{SD.Assign}(\mathcal{B T}, u)$.

2. For each $S_{i, j} \in P V_{u}$, it performs the following steps: It sets $G L=L_{i} \| d_{j}$ by obtaining $\left(L_{i}, L_{j}\right)=$ $\operatorname{Label}\left(S_{i, j}\right)$ and $d_{j}=\operatorname{Depth}\left(S_{j}\right)$, and then retrieves $\left(G L, f_{G L}(x)\right)$ from $F L$. Next, it selects random $r_{1} \in \mathbb{Z}_{N}, Y_{0}, Y_{1} \in \mathbb{G}_{p_{3}}$ and creates a personalized private key as

$$
P S K_{I D, S_{i, j}}=\left(K_{0}=g^{f_{G L}\left(L_{j}\right)}\left(u_{1}^{I D} h_{1}\right)^{r_{1}} Y_{0}, K_{1}=g^{-r_{1}} Y_{1}\right) .
$$

3. Finally, it outputs the state $S T$ and a private key $S K_{I D}=\left(P V_{u},\left\{P S K_{I D, S_{i, j}}\right\}_{S_{i, j} \in P V_{u}}\right)$.

RIBE.UpdateKey $(T, R L, M K, S T, P P)$ : This algorithm takes as input time $T$, the revocation list $R L$, the master key $M K$, the state $S T=(\mathcal{B T}, U L, F L)$, and public parameters $P P$.

1. It first defines the revoked set $R$ of user identities on the time $T$ from $R L$. That is, if there exists $\left(I D^{\prime}, T^{\prime}\right)$ such that $\left(I D^{\prime}, T^{\prime}\right) \in R L$ for any $T^{\prime} \leq T$, then $I D^{\prime} \in R$. It also defines the revoked index set $R I \subseteq \mathcal{N}$ of the revoked identity set $R$ by using $U L$. Next, it obtains $C V_{R I}=\left\{S_{i, j}\right\}$ by running $\operatorname{SD.} \operatorname{Cover}(\mathcal{B T}, R I)$.

2. For each $S_{i, j} \in C V_{R}$, it performs the following steps: It sets $G L=L_{i} \| d_{j}$ by obtaining $\left(L_{i}, L_{j}\right)=$ $\operatorname{Label}\left(S_{i, j}\right)$ and $d_{j}=\operatorname{Depth}\left(S_{j}\right)$, and then retrieves $\left(G L, f_{G L}(x)\right)$ from $F L$. Next, it selects ran$\operatorname{dom} r_{2} \in \mathbb{Z}_{N}, Y_{0}, Y_{1} \in \mathbb{G}_{p_{3}}$ and creates a time-constrained update key as

$$
T U K_{T, S_{i, j}}=\left(U_{0}=g^{f_{G L}\left(L_{j}\right)}\left(u_{2}^{T} h_{2}\right)^{r_{2}} Y_{0}, U_{1}=g^{-r_{2}} Y_{1}\right) .
$$

3. Finally, it outputs the state $S T$ and an update key $U K_{T, R}=\left(C V_{R I},\left\{T U K_{T, S_{i, j}}\right\}_{S_{i, j} \in C V_{R I}}\right)$.

RIBE.DeriveKey $\left(S K_{I D}, U K_{T, R}, P P\right)$ : This algorithm takes as input a private key $S K_{I D}=\left(P V_{u},\left\{P S K_{I D, S_{i, j}}\right\}\right)$ for an identity $I D$, an update key $U K_{T, R}=\left(C V_{R I},\left\{T U K_{T, S_{i, j}}\right\}\right)$ for time $T$ and a revoked set $R$ of identities, and the public parameters $P P$.

1. If $I D \notin R$, then it obtains $\left(S_{i, j}, S_{i^{\prime}, j^{\prime}}\right)$ by running $\operatorname{SD.Match}\left(C V_{R I}, P V_{u}\right)$ such that $S_{i, j} \in C V_{R}$, $S_{i^{\prime}, j^{\prime}} \in P V_{u}$, and $\left(L_{i}=L_{i^{\prime}}\right) \wedge\left(d_{j}=d_{j^{\prime}}\right) \wedge\left(L_{j} \neq L_{j^{\prime}}\right)$ where $\left(L_{i}, L_{j}\right)=\operatorname{Label}\left(S_{i, j}\right),\left(L_{i^{\prime}}, L_{j}^{\prime}\right)=$ $\operatorname{Label}\left(S_{i^{\prime}, j^{\prime}}\right), d_{j}=\operatorname{Depth}\left(S_{j}\right)$, and $d_{j^{\prime}}=\operatorname{Depth}\left(S_{j^{\prime}}\right)$. Otherwise, it outputs $\perp$.

2. It retrieves $T U K_{T, S_{i, j}}=\left(U_{0}, U_{1}\right)$ from $U K_{T, R}$, and $P S K_{I D, S_{i^{\prime}, j^{\prime}}}=\left(K_{0}, K_{1}\right)$ from $S K_{I D}$. Next, it sets $I=\left\{L_{j}, L_{j^{\prime}}\right\}$ and calculates two Lagrange coefficients $\Delta_{L_{j}, I}(0)$ and $\Delta_{L_{j^{\prime}}, I}(0)$ by using the fact 
$L_{j} \neq L_{j^{\prime}}$. It chooses random $r_{1}^{\prime}, r_{2}^{\prime} \in \mathbb{Z}_{N}, Y_{0}^{\prime}, Y_{1}^{\prime}, Y_{2}^{\prime} \in \mathbb{G}_{p_{3}}$ and creates decryption key components as

$$
\begin{aligned}
& D_{0}=\left(K_{0}\right)^{\Delta_{L^{\prime}, I}(0)}\left(U_{0}\right)^{\Delta_{L_{j}, I}(0)} \cdot\left(u_{1}^{I D} h_{1}\right)^{r_{1}^{\prime}}\left(u_{2}^{T} h_{2}\right)^{r_{2}^{\prime}} Y_{0}^{\prime}, \\
& D_{1}=\left(K_{1}\right)^{\Delta_{L^{\prime}, I}(0)} \cdot g^{-r_{1}^{\prime}} Y_{1}^{\prime}, D_{2}=\left(U_{1}\right)^{\Delta_{L_{j}, I}(0)} \cdot g^{-r_{2}^{\prime}} Y_{2}^{\prime} .
\end{aligned}
$$

3. Finally, it outputs a decryption key $D K_{I D, T}=\left(D_{0}, D_{1}, D_{2}\right)$.

RIBE.Encrypt $(I D, T, M, P P)$ : This algorithm takes as input an identity $I D$, time $T$, a message $M$, and the public parameters $P P$. It first chooses a random exponent $s \in \mathbb{Z}_{N}$ and outputs a ciphertext by implicitly including $I D$ and $T$ as

$$
C T_{I D, T}=\left(C=\Omega^{s} \cdot M, C_{0}=g^{s}, C_{1}=\left(u_{1}^{I D} h_{1}\right)^{s}, C_{2}=\left(u_{2}^{T} h_{2}\right)^{s}\right) .
$$

RIBE.Decrypt $\left(C T_{I D, T}, D K_{I D^{\prime}, T^{\prime}}, P P\right)$ : This algorithm takes as input a ciphertext $C T_{I D, T}=\left(C, C_{0}, C_{1}, C_{2}\right)$, a decryption key $D K_{I D^{\prime}, T^{\prime}}=\left(D_{0}, D_{1}, D_{2}\right)$, and the public parameters $P P$. If $\left(I D=I D^{\prime}\right) \wedge\left(T=T^{\prime}\right)$, then it outputs the encrypted message $M$ as $M=C \cdot\left(\prod_{i=0}^{2} e\left(C_{i}, D_{i}\right)\right)^{-1}$. Otherwise, it outputs $\perp$.

RIBE.Revoke $(I D, T, R L, S T)$ : This algorithm is the same as that of Section 3.3 .

\subsection{Security Analysis}

To prove the security of our RIBE scheme in composite-order bilinear groups, we use the dual system encryption technique of Waters [25,43]. The dual system encryption technique was successfully used to prove the security of HIBE, ABE, and PE schemes [23, 25, 34, 43]. However, the dual system encryption does not directly applicable to the RIBE scheme since the adversary of RIBE can request a private key query for a challenge identity $I D^{*}$ and an update key query for challenge time $T^{*}$ that were not allowed in IBE, HIBE, and ABE. Note that the dual system encryption technique essentially uses those restrictions of an adversary in IBE, HIBE, and ABE when it changes normal private keys to semi-functional private keys to solve the paradox of dual system encryption. To handle the private key query for $I D^{*}$ and the update key query for $T^{*}$ in RIBE, we need different techniques for dual system encryption.

To solve this problem, we first organize personalized private keys in a private key and time-constrained update keys in an update key in the order of group labels, and change those keys in the same group label from normal to semi-functional through hybrid games. Note that this strategy that changes keys in the same group label from normal to semi-functional was used in the security proof of RS-ABE [18,36]. In contrast to the RS-ABE scheme that uses the CS scheme, our RIBE scheme uses the SD scheme that has a complex key assignment part and this makes it difficult for us to prove the security. To overcome this difficulty, we carefully redesign semi-functional types and hybrid games by using the fact that there are only one private key query for $I D^{*}$ and one update key query for $T^{*}$ in RIBE that match to the challenge ciphertext.

We now consider one group label $G L$ that is associated with a polynomial $f_{G L}(x)$. This group consists of many personalized private keys and time-constrained update keys. Through another hybrid sub-games, we try to change all normal keys in this group to semi-functional keys. If an adversary only requests personalized private keys for $I D \neq I D^{*}$, then those key are first changed to nominally semi-functional keys. Next, those key are changed to semi-functional keys by using the information theoretic argument. After that, all normal time-constrained update keys are easily changed into semi-functional keys. Note that the paradox does not occur when normal time-constrained update keys are changed to semi-functional keys since the 
paradox is already solved when we change the personalized private keys and $f_{G L}(x)$ is shared. For technical reason, this process of hybrid games is more complicated. If an adversary only requests time-constrained update keys for $T \neq T^{*}$, then we also use the similar method by using nominally semi-functional keys and the information theoretic argument.

The difficult part is to handle an adversary that can request a personalized private key for $I D^{*}$ and a time-constrained update key for $T^{*}$. Let $h_{m}^{*}$ be the member index of the group $G L$ that is associated with $I D^{*}$ and $T^{*}$. Through additional hybrid games, we can change all normal personalized private keys and all normal time-constrained update keys that are not related to $h_{m}^{*}$ to semi-functional keys by introducing the nominally semi-functional type since $I D \neq I D^{*}$ and $T \neq T^{*}$ for other member index. Note that personalized private keys and time-constrained update keys for $h_{m}^{*}$ are still normal. To change those normal keys for $h_{m}^{*}$ to semi-functional keys, we embed the challenge value of the assumption into $g^{\alpha}$ instead of embedding it into $\left(u^{x} h\right)^{r}$. This method works well since there is only one member index $h_{m}^{*}$ that is left.

Note that we can solve the paradox when we change normal keys for $h_{m}^{*}$ to semi-functional keys since the Lagrange interpolation method does not work if personalized private key and time-constrained update key are related to the same member index $h_{m}^{*}$. That is, a simulator cannot derive a normal decryption key from a normal personalized private key and a normal time-constrained update key for the same $h_{m}^{*}$ since only one point of $f_{G L}(x)$ is revealed. The simulator also cannot derive a semi-functional decryption key from a semi-functional personalized private key and a semi-functional time-constrained update key for the same $h_{m}^{*}$ because of the same reason. Thus, the simulator cannot distinguish whether a personalized private key and a time-constrained update key for the same $h_{m}^{*}$ are normal of semi-functional.

Theorem 4.1. The above RIBE scheme is fully secure under chosen plaintext attacks if the SD, GSD, and ComDH assumptions hold. That is, for any PPT adversary $\mathcal{A}$, we have that $\boldsymbol{A d} \boldsymbol{v}_{R I B E \cdot \mathcal{A}}^{I N D-C P A}\left(1^{\lambda}\right) \leq \boldsymbol{A d v} v_{\mathcal{B}}^{S D}\left(1^{\lambda}\right)+$ $O\left(q^{2} \log ^{2} N_{\max }+q^{2} r_{\max }\right) \boldsymbol{A d \boldsymbol { d }} \boldsymbol{v}_{\mathcal{B}}^{G S D}\left(1^{\lambda}\right)+\boldsymbol{A d} \boldsymbol{v}_{\mathcal{B}}^{\text {ComDH }}\left(1^{\lambda}\right)$ where $q$ is the maximum number of private key, update key, and decryption key queries of $\mathcal{A}$.

Proof. We first define the semi-functional type of private keys, update keys, decryption keys, and ciphertexts. For the semi-functional type, we let $g_{2}$ denote a fixed generator of the subgroup $\mathbb{G}_{p_{2}}$.

RIBE.GenKeySF. This algorithm first creates a normal private key $S K_{I D}^{\prime}=\left(P V_{u},\left\{P S K_{I D, S_{i, j}}^{\prime}\right\}_{S_{i, j} \in P V_{u}}\right)$ by using $M K$ where $P V_{u}=\left\{S_{i, j}\right\}$ and $P S K_{I D, S_{i, j}}^{\prime}=\left(K_{0}^{\prime}, K_{1}^{\prime}\right)$. For each $S_{i, j} \in P V_{u}$, it chooses a random exponent $\delta_{i, j} \in \mathbb{Z}_{N}$ once for $S_{i, j}$ and builds a semi-functional personalized private key $P S K_{I D, S_{i, j}}=$ $\left(K_{0}=K_{0}^{\prime} g_{2}^{\delta_{i, j}}, K_{1}=K_{1}^{\prime}\right)$. It outputs a semi-functional private key $S K_{I D}=\left(P V_{u},\left\{P S K_{I D, S_{i, j}}\right\}_{S_{i, j} \in P V_{u}}\right)$.

RIBE.UpdateKeySF. This algorithm first creates a normal update key $U K_{T, R}^{\prime}=\left(C V_{R I},\left\{T U K_{T, S_{i, j}}^{\prime}\right\}_{S_{i, j} \in C V_{R I}}\right)$ by using $M K$. For each $S_{i, j} \in C V_{R I}$, it chooses a random exponent $\delta_{i, j} \in \mathbb{Z}_{N}$ once for $S_{i, j}$ and builds a semi-functional time-constrained update key $T U K_{T, S_{i, j}}=\left(U_{0}=U_{0}^{\prime} g_{2} \delta_{i, j}, U_{1}=U_{1}^{\prime}\right)$. It outputs a semifunctional update key $U K_{T, R}=\left(C V_{R I},\left\{P U K_{T, S_{i, j}}\right\}_{S_{i, j} \in C V_{R I}}\right)$.

RIBE.DeriveKeySF. This algorithm first creates a normal decryption key $D K_{I D, T}^{\prime}=\left(D_{0}^{\prime}, D_{1}^{\prime}, D_{2}^{\prime}\right)$ by using $M K$. It chooses a random exponent $a \in \mathbb{Z}_{N}$ and outputs a semi-functional decryption key $D K_{I D, T}=$ $\left(D_{0}=D_{0}^{\prime} g_{2}^{a}, D_{1}=D_{1}^{\prime}, D_{2}=D_{2}^{\prime}\right)$.

RIBE.EncryptSF. This algorithm first creates a normal ciphertext $C T_{I D, T}^{\prime}=\left(C^{\prime}, C_{0}^{\prime}, C_{1}^{\prime}, C_{2}^{\prime}\right)$. It chooses random exponents $c, d_{1}, d_{2} \in \mathbb{Z}_{N}$ and outputs semi-functional ciphertext $C T_{I D, T}=\left(C_{0}=C_{0}^{\prime} g_{2}^{c}, C_{1}=\right.$ $\left.C_{1}^{\prime} g_{2}^{c d_{1}}, C_{2}=C_{2}^{\prime} g_{2}^{c d_{2}}\right)$. 
Note that if a semi-functional decryption key is used to decrypt a semi-functional ciphertext, then the decryption fails since an additional random element $e\left(g_{2}, g_{2}\right)^{a c}$ is left.

To prove the security, we use a sequence of hybrid games. For the hybrid games that change personalized private keys (or time-constrained update keys) that are related with a subset $S_{i, j}$ from normal ones to semifunctional ones, we need to state additional information of a subset $S_{i, j}$ in $\mathcal{B T}$. Note that a personalized private key for $S_{i, j}$ and a time-constrained update key for $S_{i^{\prime}, j^{\prime}}$ share the same polynomial $f(x)$ if $\left(L_{i}=\right.$ $\left.L_{i^{\prime}}\right) \wedge\left(d_{j}=d_{j^{\prime}}\right)$ since they belong to the same group label $G L=L_{i} \| d_{j}$ where $L_{i}$ is the identifier of $v_{i}$ and $d_{j}$ is the depth of $v_{j}$. Thus we associate a personalized private key (or a time-constrained update key) with a tuple of indexes $\left(i_{g}, i_{m}, i_{c}\right)$ to state additional information about the group label $G L$ where $i_{g}$ is a group index, $i_{m}$ is a member index, and $i_{c}$ is a counter index.

Suppose that a personalized private key (or a time-constrained update key) is related with a subset $S_{i, j}$, Then this key has a group label $G L=L_{i} \| d_{j}$ and a member label $L_{j}$. The group index $i_{g}$ for personalized private keys (or time-constrained update keys) is assigned as follows: If the group label $G L$ appears first time in queries, then we set $i_{g}$ as the number of distinct group label $G L^{\prime}$ in previous queries plus one. If the group label $G L$ already appeared before in queries, then we set $i_{g}$ as the value $i_{g}^{\prime}$ of previous personalized private key (or time-constrained update key) with the same group label $G L$. The member index $i_{m}$ for the group index $i_{g}$ is assigned as follows: If the member label $L_{j}$ for this group label $G L$ appears first time in queries, then we set $i_{m}$ as the number of distinct members for this group label $G L$ in previous queries plus one. If the member label $L_{j}$ for this group label already appeared before in queries, then we set $i_{m}$ as the value $i_{m}^{\prime}$ of previous one. The counter index $i_{c}$ is assigned as follows: If the group label and member label $\left(G L, L_{j}\right)$ appears first time in queries, then we set $i_{c}$ as one. If the group label and member label $\left(G L, L_{j}\right)$ appeared before in queries, then we set $i_{c}$ as the number of queries with the group label and member label $\left(G L, L_{j}\right)$ that appeared before plus one.

The security proof consists of the sequence of hybrid games: The first game will be the original security game and the last one will be a game such that the adversary has no advantage. We define the games as follows:

Game $\mathbf{G}_{0}$. This game is the original security game. In this game, all personalized private keys, timeconstrained update keys, decryption keys, and the challenge ciphertext are normal.

Game $\mathbf{G}_{1}$. In the next game, all personalized private keys, time-constrained update keys, and decryption keys are normal, but the challenge ciphertext is semi-functional.

Game $\mathbf{G}_{2}$. Next, we define a new game $\mathbf{G}_{2}$. In this game, all personalized private keys, time-constrained update keys, and the challenge ciphertext are semi-functional, but decryption keys are normal. For the security proof, we additionally define a sequence of games $\mathbf{G}_{1,1}, \ldots, \mathbf{G}_{1, h}, \ldots, \mathbf{G}_{1, q_{g}}$ where $\mathbf{G}_{1}=\mathbf{G}_{1,0}$ and $q_{g}$ is the maximum number of group labels that are used in private keys and update keys. In the game $\mathbf{G}_{1, h}$ for $1 \leq h \leq q_{g}$, the challenge ciphertext is semi-functional, personalized private keys and time-constrained update keys with a group index $i_{g}$ such that $i_{g} \leq h$ are semi-functional, and the remaining personalized private keys and time-constrained update keys with an index $i_{g}$ such that $h<i_{g}$ are normal. It is obvious that $\mathbf{G}_{1, q_{g}}=\mathbf{G}_{2}$.

Game $\mathbf{G}_{3}$. In this game $\mathbf{G}_{3}$, all personalized private keys, time-constrained update keys, decryption keys, and the the challenge ciphertext are semi-functional.

Game $\mathbf{G}_{4}$. In the final game $\mathbf{G}_{4}$, all personalized private keys, time-constrained update keys, decryption keys, and the challenge ciphertext are semi-functional, but the challenge ciphertext component $C$ is random. 
Let $\mathbf{A d v} \mathbf{v}_{\mathcal{A}}^{G_{j}}$ be the advantage of $\mathcal{A}$ in the game $\mathbf{G}_{j}$. We easily obtain that $\operatorname{Adv}_{R I B E, \mathcal{A}}^{I N D-C P A}\left(1^{\lambda}\right)=\mathbf{A d v}_{\mathcal{A}}^{G_{0}}$, $\mathbf{A d v}_{\mathcal{A}}^{G_{1}}=\mathbf{A d v}_{\mathcal{A}}^{G_{1,0,2}}, \mathbf{A d} \mathbf{A v}_{\mathcal{A}}^{G_{2}}=\mathbf{A d} \mathbf{A v}_{\mathcal{A}}^{G_{1, q, 2}}$, and $\mathbf{A d v} \mathbf{v}_{\mathcal{A}}^{G_{4}}=0$. Through the following Lemmas 4.2. 4.3, 4.4 and 4.7. we can obtain the following equation

$$
\begin{aligned}
& \operatorname{Adv}_{R I B E, \mathcal{A}}^{I N D-C P A}\left(1^{\lambda}\right) \\
& \leq\left|\mathbf{A d v} \mathbf{A}_{\mathcal{A}}^{G_{0}}-\mathbf{A d} \mathbf{v v}_{\mathcal{A}}^{G_{1}}\right|+\sum_{h=1}^{q_{g}}\left|\mathbf{A d} \mathbf{v}_{\mathcal{A}}^{G_{1, h-1}}-\mathbf{A d v}_{\mathcal{A}}^{G_{1, h}}\right|+\left|\mathbf{A d} \mathbf{v v}_{\mathcal{A}}^{G_{2}}-\mathbf{A d v}_{\mathcal{A}}^{G_{3}}\right|+\left|\mathbf{A d} \mathbf{d v}_{\mathcal{A}}^{G_{3}}-\mathbf{A d v}_{\mathcal{A}}^{G_{4}}\right| \\
& \leq \mathbf{A d v}_{\mathcal{B}}^{S D}\left(1^{\lambda}\right)+5\left(q_{s k}+q_{u k}\right) \sum_{h=1}^{q_{g}} \sum_{h_{m}=1}^{q_{m}} \sum_{h_{c}=1}^{q_{c}} \mathbf{A d v}_{\mathcal{B}}^{G S D}\left(1^{\lambda}\right)+2 q_{d k} \mathbf{A d v}_{\mathcal{B}}^{G S D}\left(1^{\lambda}\right)+\operatorname{Adv}_{\mathcal{B}}^{C o m D H}\left(1^{\lambda}\right) \\
& \leq \mathbf{A d v}_{\mathcal{B}}^{S D}\left(1^{\lambda}\right)+\left(\frac{5}{2} q^{2} \log ^{2} N_{\max }+10 q^{2} r_{\max }+2 q\right) \mathbf{A d v}_{\mathcal{B}}^{G S D}\left(1^{\lambda}\right)+\operatorname{Adv}_{\mathcal{B}}^{C o m D H}\left(1^{\lambda}\right)
\end{aligned}
$$

where $q=q_{s k}+q_{u k}+q_{d k}$. This completes our proof.

Lemma 4.2. If the SD assumption holds, then no polynomial-time adversary can distinguish between $\boldsymbol{G}_{0}$ and $\boldsymbol{G}_{1}$ with a non-negligible advantage.

Proof. Suppose there exists an adversary $\mathcal{A}$ that distinguishes between $\mathbf{G}_{0}$ and $\mathbf{G}_{1}$ with a non-negligible advantage. A simulator $\mathcal{B}$ that solves the $\mathrm{SD}$ assumption using $\mathcal{A}$ is given: a challenge tuple $D=\left(\left(N, \mathbb{G}, \mathbb{G}_{T}, e\right)\right.$, $\left.g_{p_{1}}, g_{p_{3}}\right)$ and $Z$ where $Z=Z_{0}=X_{1} \in \mathbb{G}_{p_{1}}$ or $Z=Z_{1}=X_{1} R_{1} \in \mathbb{G}_{p_{1} p_{2}}$. Then $\mathcal{B}$ that interacts with $\mathcal{A}$ is described as follows:

Setup: $\mathcal{B}$ first chooses random exponents $u_{1}^{\prime}, h_{1}^{\prime}, u_{2}^{\prime}, h_{2}^{\prime}, \alpha \in \mathbb{Z}_{N}$. It sets $\mathcal{B T}$ by running SD.Setup and $F L$ by selecting $f_{G L}(x)$ for each $G L$ in $\mathcal{B T}$. It sets $M K=(\alpha, F L), R L=\emptyset, S T=(\mathcal{B T}, U L=\emptyset)$, and publishes $P P=\left(\left(N, \mathbb{G}, \mathbb{G}_{T}, e\right), g=g_{p_{1}}, Y=g_{p_{3}}, u_{1}=g_{p_{1}}^{u_{1}^{\prime}}, h_{1}=g_{p_{1}}^{h_{1}^{\prime}}, u_{2}=g_{p_{1}}^{u_{2}^{\prime}}, h_{2}=g_{p_{1}}^{h_{2}^{\prime}}, \Omega=e(g, g)^{\alpha}\right)$.

Phase 1: To response queries, $\mathcal{B}$ creates normal keys by running the normal algorithms since it knows $M K$. Note that it cannot create semi-functional keys since it does not know $g_{p_{2}}$.

Challenge: $\mathcal{A}$ submits a challenge identity $I D^{*}$, a challenge time $T^{*}$, and challenge messages $M_{0}^{*}, M_{1}^{*}$. $\mathcal{B}$ flips a random coin $\mu \in\{0,1\}$ and creates a challenge ciphertext $C T^{*}$ by implicitly setting $g^{s}$ to be the $\mathbb{G}_{p_{1}}$ part of $Z$ as $C T^{*}=\left(C=e(Z, g)^{\alpha} \cdot M_{\mu}^{*}, C_{0}=Z, C_{1}=(Z)^{u_{1}^{\prime} I D^{*}+h_{1}^{\prime}}, C_{2}=(Z)^{u_{2}^{\prime} T^{*}+h_{2}^{\prime}}\right)$.

Phase 2: Same as Phase 1.

Guess: $\mathcal{A}$ outputs a guess $\mu^{\prime}$. If $\mu=\mu^{\prime}$, then $\mathcal{B}$ outputs 1 . Otherwise, it outputs 0 .

If $Z=Z_{0}=X_{1}$, then the simulation is the same as $\mathbf{G}_{0}$ since the challenge ciphertext is correctly distributed. If $Z=Z_{1}=X_{1} R_{1}$, then the simulation is the same as $\mathbf{G}_{1}$ since the challenge ciphertext is generated as semi-functional by implicitly setting $d_{1} \equiv u_{1}^{\prime} I D^{*}+h_{1}^{\prime} \bmod p_{2}, d_{2} \equiv u_{2}^{\prime} T^{*}+h_{2}^{\prime} \bmod p_{2}$. The values $u_{1}^{\prime}, h_{1}^{\prime}, u_{2}^{\prime}, h_{2}^{\prime}$ modulo $p_{2}$ are not correlated with their values modulo $p_{1}$ by the Chinese Remainder Theorem (CRT). This completes our proof.

Lemma 4.3. If the GSD assumption holds, then no polynomial-time adversary can distinguish between $\boldsymbol{G}_{1, h-1}$ and $\boldsymbol{G}_{1, h}$ with a non-negligible advantage.

Proof. We first divide the behavior of an adversary as two types: Type-I and Type-II. We next show that this lemma holds for two types of the adversary. Let $I D^{*}$ and $T^{*}$ be the challenge identity and the challenge time respectively. The two types of adversaries are formally defined as follows: 
Type-I. An adversary is Type-I if it queries on an identity $I D$ such that $I D=I D^{*}$ for at least one personalized private key with the group index $h$, or it queries on time $T$ such that $T=T^{*}$ for at least one timeconstrained update key with the group index $h$. More specifically, this adversary can be divided as follows:

- Type-I-A. This adversary queries on an identity $I D$ such that $I D \neq I D^{*}$ for all personalized private keys with the group index $h$, and it queries on time $T$ such that $T=T^{*}$ for at least one time-constrained update key with the group index $h$.

- Type-I-B. This adversary queries on time $T$ such that $T \neq T^{*}$ for all time-constrained update keys with $h$, and it queries on an identity $I D$ such that $I D=I D^{*}$ for at least one personalized private key with $h$.

- Type-I-C. This adversary queries on an identity $I D$ such that $I D=I D^{*}$ for at least one personalized private key with $h$, and it queries on time $T$ such that $T=T^{*}$ for at least one timeconstrained update key with $h$.

Type-II. An adversary is Type-II if it queries on an identity $I D$ such that $I D \neq I D^{*}$ for all personalized private keys with the group index $h$, and it queries on time $T$ such that $T \neq T^{*}$ for all time-constrained update keys with the group index $h$.

Let $C V_{R I^{*}}$ be the covering set of the update key for the time $T^{*}$ and revoked set $R^{*}$, and $P V_{u^{*}}$ be the private set of the private key for the identity $I D^{*}$. Let $h_{m}^{*}$ be a member index of the group index $h$ such that the personalized private key for $I D^{*}$ or the time-constrained update key for $T^{*}$ belong to the member index $h_{m}^{*}$. If the adversary is Type-I-A, then there is only one member index $h_{m}^{*}$ since $C V_{R I^{*}}$ is a partition. If the adversary is In Type-I-B, then there is only one member index $h_{m}^{*}$ since $P V_{u^{*}}$ is related with a path. If the adversary is Type-I-C, the member index $h_{m}^{*}$ of $C V_{R I^{*}}$ with the group index $h$ should be the same as that of $P V_{u^{*}}$ with the same group index $h$ in the SD scheme if $I D^{*} \in R^{*}$. If the adversary is Type-II, then there is no member index $h_{m}^{*}$ since the adversary does not request a key query for $I D^{*}$ or $T^{*}$.

For the Type-I adversary $\mathcal{A}_{I}$, we define hybrid games $\mathbf{H}_{(1,1), 1}, \mathbf{H}_{(1,1), 2}, \ldots, \mathbf{H}_{\left(q_{m}, q_{c}\right), 1}, \mathbf{H}_{\left(q_{m}, q_{c}\right), 2}=\mathbf{H}_{\left(q_{m}, q_{c}\right), 2}^{\prime}$, $\mathbf{H}_{\left(q_{m}, q_{c}\right), 1}^{\prime}, \ldots, \mathbf{H}_{(1,1), 2}^{\prime}, \mathbf{H}_{(1,1), 1}^{\prime}, \mathbf{H}_{(1,0), 2}^{\prime}, \mathbf{H}^{\prime \prime}$ where $\mathbf{G}_{1, h-1}=\mathbf{H}_{(1,0), 2}, \mathbf{H}^{\prime \prime}=\mathbf{G}_{1, h}, q_{m}$ is the maximum number of distinct member subsets of the group index $h$, and $q_{c}$ is the maximum number of queries for one member subset. The games are formally defined as follows:

Game $\mathbf{H}_{\left(h_{m}, h_{c}\right), 1}$. This game $\mathbf{H}_{\left(h_{m}, h_{c}\right), 1}$ for $1 \leq h_{m} \leq q_{m}$ and $1 \leq h_{c} \leq q_{c}$ is almost the same as $\mathbf{G}_{1, h-1}$ except the generation of personalized private keys and time-constrained update keys with the group index $h$. These personalized private keys and time-constrained update keys with indexes $\left(i_{g}=h, i_{m}, i_{c}\right)$ are generated as follows:

- Case $i_{g}<h$ : The keys (personalized private keys and time-constrained update keys) are generated as semi-functional.

- Case $i_{g}=h$ : The keys are generated as follows:

$-\left(i_{m} \neq h_{m}^{*}\right) \wedge\left(i_{m}<h_{m}\right)$ or $\left(i_{m} \neq h_{m}^{*}\right) \wedge\left(i_{m}=h_{m}\right) \wedge\left(i_{c}<h_{c}\right):$

If this is a personalized private key query, then it generates a normal $P S K^{\prime}=\left(K_{0}^{\prime}, K_{1}^{\prime}\right)$ and creates the semi-functional personalized private key of type 2 as $P S K_{I D, S_{i, j}}=\left(K_{0}=\right.$ $\left.K_{0}^{\prime} g_{2}^{a}, K_{1}=K_{1}^{\prime}\right)$ by selecting a new random exponent $a \in \mathbb{Z}_{N}$.

If this is a time-constrained update key query, then it generates a normal $T U K^{\prime}=\left(U_{0}^{\prime}, U_{1}^{\prime}\right)$ and creates the semi-functional time-constrained update key of type 2 as $T U K_{T, S_{i, j}}=\left(U_{0}=\right.$ $\left.U_{0}^{\prime} g_{2}^{a}, U_{1}=U_{1}^{\prime}\right)$ by selecting a new random exponent $a \in \mathbb{Z}_{N}$. 
- $\left(i_{m} \neq h_{m}^{*}\right) \wedge\left(i_{m}=h_{m}\right) \wedge\left(i_{c}=h_{c}\right)$ :

If this is a personalized private key query, then it generates a normal $P S K^{\prime}=\left(K_{0}^{\prime}, K_{1}^{\prime}\right)$ and creates the semi-functional personalized private key of type 1 as $P S K_{I D, S_{i, j}}=\left(K_{0}=\right.$ $\left.K_{0}^{\prime} g_{2}^{a}, K_{1}=K_{1}^{\prime} g_{2}^{b}\right)$ by selecting new random exponents $a, b \in \mathbb{Z}_{N}$.

If this is a time-constrained update key query, then it generates a normal $T U K^{\prime}=\left(U_{0}^{\prime}, U_{1}^{\prime}\right)$ and creates the semi-functional time-constrained update key of type 1 as $T U K_{T, S_{i, j}}=\left(U_{0}=\right.$ $\left.U_{0}^{\prime} g_{2}^{a}, U_{1}=U_{1}^{\prime} g_{2}^{b}\right)$ by selecting new random exponents $a, b \in \mathbb{Z}_{N}$.

- $\left(i_{m} \neq h_{m}^{*}\right) \wedge\left(i_{m}=h_{m}\right) \wedge\left(h_{c}<i_{c}\right)$ or $\left(i_{m} \neq h_{m}^{*}\right) \wedge\left(h_{m}<i_{m}\right)$ : It simply creates a normal type key.

- $\left(i_{m}=h_{m}^{*}\right):$ It simply creates a normal type key.

- Case $i_{g}>h$ : The keys are generated as normal.

Note that if a semi-functional personalized private key of type 1 and a normal time-constrained update key are used to decrypt a semi-functional ciphertext, then the decryption fails since an additional random element $e\left(g_{2}, g_{2}\right)^{c\left(a-b d_{1}\right)}$ is left. If $a=b d_{1}$, then the decryption succeeds and this personalized private key is nominally semi-functional of type 1. Similarly, if a semi-functional time-constrained update key of type 1 and a normal personalized private key are used to decrypt a semi-functional ciphertext, then the decryption fails since an additional random element $e\left(g_{2}, g_{2}\right)^{c\left(a-b d_{1}\right)}$ is left. If $a=$ $b d_{1}$, then the decryption succeeds and this time-constrained update key is nominally semi-functional of type 1 .

Game $\mathbf{H}_{\left(h_{m}, h_{c}\right), 2}$. This game $\mathbf{H}_{\left(h_{m}, h_{c}\right), 2}$ is almost the same as $\mathbf{H}_{\left(h_{m}, h_{c}\right), 1}$ except that the personalized private key (or the time-constrained update key) with the indexes $\left(i_{g}=h, i_{m}, i_{c}\right)$ such that $\left(i_{m} \neq h_{m}^{*}\right) \wedge\left(i_{m}=\right.$ $\left.h_{m}\right) \wedge\left(i_{c}=h_{c}\right)$ is generated with $b=0$. In the game $\mathbf{H}_{\left(q_{m}, q_{c}\right), 2}$, all personalized private keys and timeconstrained update keys with the group index $h$ are semi-functional of type 2 except that personalized private keys and time-constrained update keys with the member index $h_{m}^{*}$ are normal.

Game $\mathbf{H}_{\left(h_{m}, h_{c}\right), 1}^{\prime}$. This game $\mathbf{H}_{\left(h_{m}, h_{c}\right), 1}^{\prime}$ is almost the same as $\mathbf{H}_{\left(h_{m}, h_{c}\right), 1}$ except the generation of a personalized private key (or a time-constrained update key) with the indexes $\left(i_{g}=h, i_{m}, i_{c}\right)$ such that $\left(i_{m} \neq h_{m}^{*}\right) \wedge$ $\left(i_{m}=h_{m}\right) \wedge\left(h_{c} \leq i_{c}\right)$ or $\left(i_{m} \neq h_{m}\right) \wedge\left(h_{m}<i_{m}\right)$. These personalized private keys (or time-constrained update keys) are generated as follows:

- $\left(i_{m} \neq h_{m}^{*}\right) \wedge\left(i_{m}=h_{m}\right) \wedge\left(i_{c}=h_{c}\right)$ : Let $\delta_{i, j}$ be a random exponent in $\mathbb{Z}_{N}$ that is fixed for this member subset $S_{i, j}$.

If this is a personalized private key query, then it generates $P S K^{\prime \prime}=\left(K_{0}^{\prime \prime}, K_{1}^{\prime \prime}\right)$ as the same as $\mathbf{H}_{\left(h_{m}, h_{c}\right), 1}$ and creates the semi-functional personalized private key as $P S K_{I D, S_{i, j}}=\left(K_{0}=\right.$ $\left.K_{0}^{\prime \prime} g_{2}^{\delta_{i, j}}, K_{1}=K_{1}^{\prime \prime}\right)$.

If this is a time-constrained update key query, then it generates $T U K^{\prime \prime}=\left(U_{0}^{\prime \prime}, U_{1}^{\prime \prime}\right)$ as the same as $\mathbf{H}_{\left(h_{m}, h_{c}\right), 1}$ and creates the semi-functional time-constrained update key as $T U K_{T, S_{i, j}}=\left(U_{0}=\right.$ $\left.U_{0}^{\prime \prime} g_{2}^{\delta_{i, j}}, U_{1}=U_{1}^{\prime \prime}\right)$.

- $\left(i_{m} \neq h_{m}^{*}\right) \wedge\left(i_{m}=h_{m}\right) \wedge\left(h_{c}<i_{c}\right)$ or $\left(i_{m} \neq h_{m}^{*}\right) \wedge\left(h_{m}<i_{m}\right)$ : It creates a semi-functional key by using the fixed $\delta_{i, j}$ for this member subset $S_{i, j}$.

Game $\mathbf{H}_{\left(h_{m}, h_{c}\right), 2}^{\prime}$. This game $\mathbf{H}_{\left(h_{m}, h_{c}\right), 2}^{\prime}$ is almost the same as $\mathbf{H}_{\left(h_{m}, h_{c}\right), 1}^{\prime}$ except that the personalized private key or time-constrained update key with the indexes $\left(i_{g}=h, i_{m}, i_{c}\right)$ such that $\left(i_{m} \neq h_{m}^{*}\right) \wedge\left(i_{m}=h_{m}\right) \wedge$ 
$\left(i_{c}=h_{c}\right)$ is generated with $b=0$. The modification is similar to the game $\mathbf{H}_{\left(h_{m}, h_{c}\right), 1}^{\prime}$. In the game $\mathbf{H}_{(1,0), 2}^{\prime}$, all personalized private keys and all time-constrained update keys with the group index $h$ except the keys with the member index $h_{m}^{*}$ are semi-functional where a fixed $\delta_{i, j}$ is used for each member.

Game $\mathbf{H}^{\prime \prime}$. This game $\mathbf{H}^{\prime \prime}$ is the same as $\mathbf{G}_{1, h}$. Compared to the game $\mathbf{H}_{(1,0), 2}^{\prime}$, the remaining personalized private keys and time-constrained update keys with the member index $h_{m}^{*}$ are changed to be semifunctional by using a fixed $\delta_{i, j}$ for this member subset $S_{i, j}$.

Let $\mathbf{A d} \mathbf{d v}_{\mathcal{A}_{I}}^{H_{i}}$ be the advantage of $\mathcal{A}_{I}$ in a game $\mathbf{H}_{i}$. From the following Lemmas $4.8,4.9,4.10,4.11$, and 4.12, we can obtain the following equation

$$
\begin{aligned}
& \mathbf{A d v}_{\mathcal{A}_{I}}^{H_{(1,0), 2}}-\mathbf{A d v}_{\mathcal{A}_{I}}^{H^{\prime \prime}} \\
& \leq \sum_{h_{m}=1}^{q_{m}} \sum_{h_{c}=1}^{q_{c}}\left|\mathbf{A d d}{ }_{\mathcal{A}_{I}}^{H_{\left(h_{m}, h_{c}-1\right), 2}}-\mathbf{A d d v}{ }_{\mathcal{A}_{I}}^{H_{\left(h_{m}, h_{c}\right), 1}}\right|+\sum_{h_{m}=1}^{q_{m}} \sum_{h_{c}=1}^{q_{c}}\left|\mathbf{A d} \mathbf{d v}_{\mathcal{A}_{I}}^{H_{\left(h_{m}, h_{c}\right), 1}}-\mathbf{A d v}_{\mathcal{A}_{I}}^{H_{\left(h_{m}, h_{c}\right), 2}}\right|+
\end{aligned}
$$

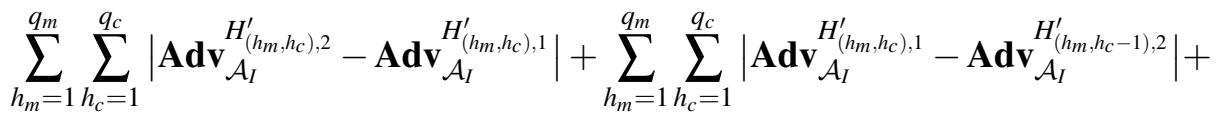

$$
\begin{aligned}
& \left|\mathbf{A d} \mathbf{v v}_{\mathcal{A}_{I}}^{H_{(1,0), 2}^{\prime}}-\mathbf{A d} \mathbf{d} \mathbf{v}_{\mathcal{A}_{I}}^{H^{\prime \prime}}\right| \\
& \leq 5\left(q_{s k}+q_{u k}\right) \sum_{h_{m}=1}^{q_{m}} \sum_{h_{c}=1}^{q_{c}} \mathbf{A d v}_{\mathcal{B}}^{G S D}\left(1^{\lambda}\right)
\end{aligned}
$$

For the Type-II adversary $\mathcal{A}_{I I}$, we define hybrid games $\mathbf{I}_{(1,1), 1}, \mathbf{I}_{(1,1), 2}, \ldots, \mathbf{I}_{\left(q_{m}, q_{c}\right), 1}, \mathbf{I}_{\left(q_{m}, q_{c}\right), 2}=\mathbf{I}_{\left(q_{m}, q_{c}\right), 2}^{\prime}$, $\mathbf{I}_{\left(q_{m}, q_{c}\right), 1}^{\prime}, \ldots, \mathbf{I}_{(1,1), 2}^{\prime}, \mathbf{I}_{(1,1), 1}^{\prime}, \mathbf{I}_{(1,0), 2}^{\prime}, \mathbf{I}^{\prime \prime}$ where $\mathbf{G}_{1, h-1}=\mathbf{I}_{(1,0), 2}$ and $\mathbf{I}_{(1,0), 2}^{\prime}=\mathbf{I}^{\prime \prime}=\mathbf{G}_{1, h}$. The games are formally defined as follows:

Game $\mathbf{I}_{\left(h_{m}, h_{c}\right), 1}$. This game $\mathbf{I}_{\left(h_{m}, h_{c}\right), 1}$ is almost the same as $\mathbf{H}_{\left(h_{m}, h_{c}\right), 1}$ except that there is no case $i_{m}=h_{m}^{*}$ since the adversary is Type-II.

Game $\mathbf{I}_{\left(h_{m}, h_{c}\right), 2}$. This game $\mathbf{I}_{\left(h_{m}, h_{c}\right), 2}$ is almost the same as $\mathbf{H}_{\left(h_{m}, h_{c}\right), 2}$ except that there is no case $i_{m}=h_{m}^{*}$ since the adversary is Type-II.

Game $\mathbf{I}_{\left(h_{m}, h_{c}\right), 1}^{\prime}$. This game $\mathbf{I}_{\left(h_{m}, h_{c}\right), 1}^{\prime}$ is almost the same as $\mathbf{H}_{\left(h_{m}, h_{c}\right), 1}^{\prime}$ except that there is no case $i_{m}=h_{m}^{*}$ since the adversary is Type-II.

Game $\mathbf{I}_{\left(h_{m}, h_{c}\right), 2}^{\prime}$. This game $\mathbf{I}_{\left(h_{m}, h_{c}\right), 1}^{\prime}$ is almost the same as $\mathbf{H}_{\left(h_{m}, h_{c}\right), 1}^{\prime}$ except that there is no case $i_{m}=h_{m}^{*}$ since the adversary is Type-II. In the game $\mathbf{I}_{(1,0), 2}^{\prime}$, all personalized private keys and all time-constrained update keys with the group index $h$ are semi-functional where a fixed $\delta_{i, j}$ is used for each member.

Let $\mathbf{A d} \mathbf{v}_{\mathcal{A}_{I I}}^{I_{i}}$ be the advantage of $\mathcal{A}_{I I}$ in a game $\mathbf{I}_{i}$. From the following Lemmas 4.13, 4.14, 4.15, and 4.16 we can easily obtain the equation $\mathbf{A d v}_{\mathcal{A}_{I I}}^{I_{0,2}}-\mathbf{A d v}_{\mathcal{A}_{I I}}^{I^{\prime \prime}} \leq 5\left(q_{s k}+q_{u k}\right) \mathbf{A d v}_{\mathcal{B}}^{G S D}\left(1^{\lambda}\right)$.

Let $E_{I}, E_{I I}$ be the event such that an adversary behave like the Type-I, Type-II adversary respectively. 
From the above three inequalities for three types, we have the following inequality as

$$
\begin{aligned}
& \mathbf{A d v} \mathbf{v}_{\mathcal{A}}^{G_{1, h-1}}-\mathbf{A d v}_{\mathcal{A}}^{G_{1, h}} \leq \operatorname{Pr}\left[E_{I}\right]\left(\mathbf{A d v}_{\mathcal{A}_{I}}^{G_{1, h-1}}-\mathbf{A d} \mathbf{d v}_{\mathcal{A}_{I}}^{G_{1, h}}\right)+\operatorname{Pr}\left[E_{I I}\right]\left(\mathbf{A d v}_{\mathcal{A}_{I I}}^{G_{1, h-1}}-\mathbf{A d v}_{\mathcal{A}_{I I}}^{G_{1, h}}\right) \\
& \leq \operatorname{Pr}\left[E_{I}\right]\left(\mathbf{A d v}_{\mathcal{A}_{I}}^{H_{(1,0), 2}}-\mathbf{A d v}_{\mathcal{A}_{I}}^{H^{\prime \prime}}\right)+\operatorname{Pr}\left[E_{I I}\right]\left(\mathbf{A d v}_{\mathcal{A}_{I I}}^{I_{(1,0), 2}}-\mathbf{A d v}_{\mathcal{A}_{I I}}^{I^{\prime \prime}}\right) \\
& \leq 5\left(q_{s k}+q_{u k}\right) \sum_{h_{m}=1}^{q_{m}} \sum_{h_{c}=1}^{q_{c}}\left(\operatorname{Pr}\left[E_{I}\right] \mathbf{A d v}_{\mathcal{B}}^{G S D}\left(1^{\lambda}\right)+\operatorname{Pr}\left[E_{I I}\right] \mathbf{A d v}_{\mathcal{B}}^{G S D}\left(1^{\lambda}\right)\right) \\
& \leq 5\left(q_{s k}+q_{u k}\right) \sum_{h_{m}=1}^{q_{m}} \sum_{h_{c}=1}^{q_{c}} \mathbf{A d v}_{\mathcal{B}}^{G S D}\left(1^{\lambda}\right)
\end{aligned}
$$

This completes our proof.

Lemma 4.4. If the GSD assumption holds, then no polynomial-time adversary can distinguish between $\boldsymbol{G}_{2}$ and $\boldsymbol{G}_{3}$ with a non-negligible advantage.

Proof. Let $q_{d k}$ be the number of decryption key queries of an adversary. For the security proof, we additionally define a sequence of games $\mathbf{G}_{2,1,1}, \mathbf{G}_{2,1,2}, \ldots, \mathbf{G}_{2, k, 1}, \mathbf{G}_{2, k, 2}, \ldots, \mathbf{G}_{2, q_{d k}, 1}, \mathbf{G}_{2, q_{d k}, 2}$ where $\mathbf{G}_{2}=\mathbf{G}_{2,0,2}$. The games are defined as follows:

Game $\mathbf{G}_{2, k, 1}$. In this game, all personalized private keys, all time-constrained update keys, and the challenge ciphertext are semi-functional. The first $k-1$ decryption keys are semi-functional. The $k$ th decryption key is semi-functional of type 1 and it is generated as $D K_{I D, T}=\left(D_{0}=D_{0}^{\prime} g_{2}^{a}, D_{1}=D_{1}^{\prime} g_{2}^{-b_{1}}, D_{2}=\right.$ $\left.D_{2}^{\prime} g_{2}^{-b_{2}}\right)$ where $D K_{I D, T}^{\prime}=\left(D_{0}^{\prime}, D_{1}^{\prime}, D_{2}^{\prime}\right)$ is a normal decryption key and $a, b_{1}, b_{2}$ are random exponents in $\mathbb{Z}_{N}$. The remaining decryption keys are normal.

Game $\mathbf{G}_{2, k, 2}$. In this game, all personalized private keys, all time-constrained update keys, the challenge ciphertext header, and the first $k$ decryption keys are semi-functional. But the remaining decryption keys are normal. It is obvious that $\mathbf{G}_{2, q_{d k}, 2}=\mathbf{G}_{3}$.

Note that if a semi-functional decryption key of type 1 is used to decrypt a semi-functional ciphertext, then the decryption fails since an additional random element $e\left(g_{2}, g_{2}\right)^{c\left(a-b_{1} d_{1}-b_{2} d_{2}\right)}$ is left. If $a=b_{1} d_{1}+b_{2} d_{2}$, then the decryption succeeds. In this case, we say that the decryption key is nominally semi-functional of type 1 .

Let $\mathbf{A d v}_{\mathcal{A}}^{G_{2, i, j}}$ be the advantage of $\mathcal{A}$ in the game $\mathbf{G}_{2, i, j}$. We easily obtain that $\mathbf{A d v}_{\mathcal{A}}^{G_{2}}=\mathbf{A d v}_{\mathcal{A}}^{G_{2,0,2}}$ and $\mathbf{A d v}_{\mathcal{A}}^{G_{3}}=\operatorname{Adv}_{\mathcal{A}}^{G_{2, q_{d k}, 2}}$. From the following Lemmas 4.5 and 4.6 , we can obtain the following equation

$$
\begin{aligned}
\mathbf{A d v}_{\mathcal{A}}^{G_{2}}-\mathbf{A d v}_{\mathcal{A}}^{G_{3}} & \leq \sum_{k=1}^{q_{d k}}\left|\mathbf{A d} \mathbf{d v}_{\mathcal{A}}^{G_{2, k-1,2}}-\mathbf{A d} \mathbf{v}_{\mathcal{A}}^{G_{2, k, 1}}\right|+\sum_{k=1}^{q_{d k}}\left|\mathbf{A d} \mathbf{d v}_{\mathcal{A}}^{G_{2, k, 1}}-\mathbf{A d} \mathbf{v}_{\mathcal{A}}^{G_{2, k, 2}}\right| \\
& \leq 2 q_{d k} \mathbf{A d v}_{\mathcal{B}}^{G S D}\left(1^{\lambda}\right) .
\end{aligned}
$$

This completes our proof.

Lemma 4.5. If the GSD assumption holds, then no polynomial-time adversary can distinguish between $\boldsymbol{G}_{2, k-1,2}$ and $\boldsymbol{G}_{2, k, 1}$ with a non-negligible advantage.

Proof. Suppose there exists an adversary $\mathcal{A}$ that distinguishes between $\mathbf{G}_{2, k-1,2}$ and $\mathbf{G}_{2, k, 1}$ with a nonnegligible advantage. A simulator $\mathcal{B}$ that solves the GSD assumption using $\mathcal{A}$ is given: a challenge tuple 
$D=\left(\left(N, \mathbb{G}, \mathbb{G}_{T}, e\right), g_{p_{1}}, g_{p_{3}}, X_{1} R_{1}, R_{2} Y_{1}\right)$ and $Z$ where $Z=Z_{0}=X_{2} Y_{2}$ or $Z=Z_{1}=X_{2} R_{3} Y_{2}$. Then $\mathcal{B}$ that interacts with $\mathcal{A}$ is described as follows:

Setup: $\mathcal{B}$ first chooses random exponents $u_{1}^{\prime}, h_{1}^{\prime}, u_{2}^{\prime}, h_{2}^{\prime}, \alpha \in \mathbb{Z}_{N}$. It sets $\mathcal{B} \mathcal{T}$ by running SD.Setup and $F L$ by selecting $f_{G L}(x)$ for each $G L$ in $\mathcal{B T}$. It sets $M K=(\alpha, F L), R L=\emptyset, S T=(\mathcal{B T}, U L=\emptyset)$, and publishes $P P=\left(\left(N, \mathbb{G}, \mathbb{G}_{T}, e\right), g=g_{p_{1}}, Y=g_{p_{3}}, u_{1}=g_{p_{1}}^{u_{1}^{\prime}}, h_{1}=g_{p_{1}}^{h_{1}^{\prime}}, u_{2}=g_{p_{1}}^{u_{2}^{\prime}}, h_{2}=g_{p_{1}}^{h_{2}^{\prime}}, \Omega=e(g, g)^{\alpha}\right)$.

Phase 1: For each query, $\mathcal{B}$ proceeds as follows: If this is a personalized private key (or time-constrained update key) query, then it creates a semi-functional one by using $M K$ and $R_{2} Y_{1}$ given in the assumption. If this is a $j$ th decryption key query for $I D$ and $T$, then it handles this query as follows:

- If $j<k$, then it creates a semi-functional decryption key since it knows $M K$ and $R_{2} Y_{1}$ is given in the assumption.

- If $j=k$, then it selects random $r_{1}^{\prime}, r_{2}^{\prime} \in \mathbb{Z}_{N}, Y_{0}^{\prime}, Y_{1}^{\prime}, Y_{2}^{\prime} \in \mathbb{G}_{p_{3}}$ and creates a decryption key $D K_{I D, T}=$ $\left(D_{0}=g^{\alpha}(Z)^{\left(u_{1}^{\prime} I D+h_{1}^{\prime}\right) r_{1}^{\prime}}(Z)^{\left(u_{2}^{\prime} T+h_{2}^{\prime}\right) r_{2}^{\prime}} Y_{0}^{\prime}, D_{1}=(Z)^{-r_{1}^{\prime}} Y_{1}^{\prime}, D_{2}=(Z)^{-r_{2}^{\prime}} Y_{2}^{\prime}\right)$.

- If $j>k$, then it creates a normal decryption key since it knows $M K$.

Challenge: $\mathcal{B}$ flips a random coin $\mu \in\{0,1\}$ and creates a semi-functional ciphertext by implicitly setting $g^{s}=X_{1}$ and $g_{2}^{c}=R_{1}$ as $C T^{*}=\left(C=e\left(X_{1} R_{1}, g\right)^{\alpha} \cdot M_{\mu}^{*}, C_{0}=X_{1} R_{1}, C_{1}=\left(X_{1} R_{1}\right)^{u_{1}^{\prime} I D^{*}+h_{1}^{\prime}}, C_{2}=\left(X_{1} R_{1}\right)^{u_{2}^{\prime} T^{*}+h_{2}^{\prime}}\right)$.

Phase 2: Same as Phase 1.

Guess: $\mathcal{A}$ outputs a guess $\mu^{\prime}$. If $\mu=\mu^{\prime}$, then $\mathcal{B}$ outputs 1 . Otherwise, it outputs 0 .

If $Z=Z_{0}=X_{2} Y_{2}$, then the simulation is the same as $\mathbf{G}_{2, k-1,2}$ since the $k$ th decryption key and the semi-functional challenge ciphertext are correctly distributed by implicitly setting $r_{1} \equiv \log _{g}\left(X_{2}\right) r_{1}^{\prime} \bmod p_{1}$, $r_{2} \equiv \log _{g}\left(X_{2}\right) r_{2}^{\prime} \bmod p_{1}$, and $s \equiv \log _{g}\left(X_{1}\right) \bmod p_{1}$. If $Z=Z_{1}=X_{2} R_{3} Y_{2}$, then the simulation is almost the same as $\mathbf{G}_{2, k, 1}$ except that the $k$ th decryption key is generated as a nominally semi-functional one of type 1 by implicitly setting $b_{1} \equiv \log _{g_{2}}\left(R_{3}\right) r_{1}^{\prime} \bmod p_{2}, b_{2} \equiv \log _{g_{2}}\left(R_{3}\right) r_{2}^{\prime} \bmod p_{2}$, and $a \equiv b_{1}\left(u_{1}^{\prime} I D+h_{1}^{\prime}\right)+$ $b_{2}\left(u_{2}^{\prime} T+h_{2}^{\prime}\right) \bmod p_{2}$. Note that we solve the paradox of the dual system encryption by introducing the nominally semi-functional decryption key which is similar to that of Lewko and Waters [25]. That is, the simulator cannot distinguish whether the $k$ th decryption key is normal or nominally semi-functional since the nominally semi-functional one is correlated to the challenge ciphertext.

To finish the proof, we should argue that the adversary cannot distinguish the nominally semi-functional decryption key from the semi-functional decryption key of type 1. For this argument, we show an information theoretic argument by using the restriction of the security model such that a decryption key query for the identity $I D^{*}$ and the time $T^{*}$ is not allowed. Suppose there exists an unbounded adversary. Then the adversary can gather the values $a \equiv b_{1}\left(u_{1}^{\prime} I D+h_{1}^{\prime}\right)+b_{2}\left(u_{2}^{\prime} T+h_{2}^{\prime}\right), b_{1}, b_{2} \bmod p_{2}$ from the $k$ th decryption key and $d_{1} \equiv u_{1}^{\prime} I D^{*}+h_{1}^{\prime}, d_{2} \equiv u_{2}^{\prime} T^{*}+h_{2}^{\prime} \bmod p_{2}$ from the challenge ciphertext. If $\left(I D \neq I D^{*}\right) \vee\left(T \neq T^{*}\right)$, then $b_{1}\left(u_{1}^{\prime} I D+h_{1}^{\prime}\right)+b_{2}\left(u_{2}^{\prime} T+h_{2}^{\prime}\right) \bmod p_{2}, u_{1}^{\prime} I D^{*}+h_{1}^{\prime} \bmod p_{2}$ and $u_{2}^{\prime} T^{*}+h_{2}^{\prime} \bmod p_{2}$ look random to the adversary since $u_{i}^{\prime} x+h_{i}^{\prime}$ is a pair-wise independent function, $\left(I D \neq I D^{*}\right) \vee\left(T \neq T^{*}\right)$ by the restriction of the security model, and $u_{1}^{\prime}, h_{1}^{\prime}, u_{2}^{\prime}, h_{2}^{\prime} \bmod p_{2}$ are information theoretically hidden to the adversary. This completes our proof.

Lemma 4.6. If the GSD assumption holds, then no polynomial-time adversary can distinguish between $\boldsymbol{G}_{2, k, 1}$ and $\boldsymbol{G}_{2, k, 2}$ with a non-negligible advantage.

Proof. The proof of this lemma is almost the same as that of Lemma 4.5, except the generation of the $k$ th decryption key. The $k$ th decryption key for $I D$ and $T$ is generated as follows: 
- If $j=k$, then it selects random $r_{1}^{\prime}, r_{2}^{\prime}, a^{\prime} \in \mathbb{Z}_{N}, Y_{0}^{\prime}, Y_{1}^{\prime}, Y_{2}^{\prime} \in \mathbb{G}_{p_{3}}$ and creates a decryption key $D K_{I D, T}=$ $\left(D_{0}=g^{\alpha}(Z)^{\left(u_{1}^{\prime} I D+h_{1}^{\prime}\right) r_{1}^{\prime}}(Z)^{\left(u_{2}^{\prime} T+h_{2}^{\prime}\right) r_{2}^{\prime}}\left(R_{2} Y_{1}\right)^{a^{\prime}} Y_{0}^{\prime}, D_{1}=(Z)^{-r_{1}^{\prime}} Y_{1}^{\prime}, D_{2}=(Z)^{-r_{2}^{\prime}} Y_{2}^{\prime}\right)$.

Note that the $k$ th decryption key is no longer correlated with $C T^{*}$ since the element $D_{0}$ is re-randomized by $\left(R_{2} Y_{1}\right)^{a^{\prime}}$. If $Z=Z_{0}=X_{2} Y_{2}$, then the simulation is the same as $\mathbf{G}_{2, k, 2}$. If $Z=Z_{1}=X_{2} R_{3} Y_{2}$, then the simulation is the same as $\mathbf{G}_{2, k, 1}$.

Lemma 4.7. If the ComDH assumption holds, then no polynomial-time adversary can distinguish between $\boldsymbol{G}_{3}$ and $\boldsymbol{G}_{4}$ with a non-negligible advantage.

Proof. Suppose there exists an adversary $\mathcal{A}$ that distinguish $\mathbf{G}_{3}$ from $\mathbf{G}_{4}$ with a non-negligible advantage. A simulator $\mathcal{B}$ that solves the ComDH assumption using $\mathcal{A}$ is given: a challenge tuple $D=\left(\left(N, \mathbb{G}, \mathbb{G}_{T}, e\right)\right.$, $\left.g_{p_{1}}, g_{p_{2}}, g_{p_{3}}, g_{p_{1}}^{a} R_{1}, g_{p_{1}}^{b} R_{2}\right)$ and $Z$ where $Z=Z_{0}=e\left(g_{p_{1}}, g_{p_{1}}\right)^{a b}$ or $Z=Z_{1}=e\left(g_{p_{1}}, g_{p_{1}}\right)^{c}$. Then $\mathcal{B}$ that interacts with $\mathcal{A}$ is described as follows:

Setup: $\mathcal{B}$ chooses random exponents $u_{1}^{\prime}, h_{1}^{\prime}, u_{2}^{\prime}, h_{2}^{\prime} \in \mathbb{Z}_{N}$ and implicitly sets $\alpha=a$ from the term $g_{p_{1}}^{a} R_{1}$. It sets $\mathcal{B T}$ by running SD.Setup and $F L$ by selecting a random point $(x, y)$ for each $G L$ in $B T$. Note that a random $f_{G L}(x)$ for $G L$ is implicitly defined by two points $(0, a)$ and $(x, y)$ and $g_{p_{1}}^{f_{G L}\left(L_{j}\right)} R$ for any $L_{j}$ can be computable from the Lagrange interpolation method where $R \in \mathbb{G}_{p_{2}}$. It sets $R L=\emptyset, S T=(\mathcal{B T}, U L=\emptyset)$ and publishes the public parameters $P P=\left(\left(N, \mathbb{G}, \mathbb{G}_{T}, e\right), g=g_{p_{1}}, Y=g_{p_{3}}, u_{1}=g^{u_{1}^{\prime}}, h_{1}=g^{h_{1}^{\prime}}, u_{2}=g^{u_{2}^{\prime}}, h_{2}=\right.$ $\left.g^{h_{2}^{\prime}}, \Omega=e\left(g, g_{p_{1}}^{a} R_{1}\right)\right)$.

Phase 1: For each query, $\mathcal{B}$ creates a semi-functional key since $g_{p_{2}}^{a} R_{1}$ and $g_{p_{2}}$ are given from the assumption. Note that it cannot create a normal update key since it does not know $g_{p_{1}}^{a} \in \mathbb{G}_{p_{1}}$.

Challenge: $\mathcal{B}$ first flips a random coin $\mu \in\{0,1\}$ and creates a challenge ciphertext $C T^{*}=\left(C=Z \cdot M_{\mu}^{*}, C_{0}=\right.$ $\left.g_{p_{1}}^{b} R_{2}, C_{1}=\left(g_{p_{1}}^{b} R_{2}\right)^{u_{1}^{\prime} I D^{*}+h_{1}^{\prime}}, C_{2}=\left(g_{p_{1}}^{b} R_{2}\right)^{u_{2}^{\prime} T^{*}+h_{2}^{\prime}}\right)$.

Phase 2: Same as Phase 1 .

Guess: $\mathcal{A}$ outputs a guess $\mu^{\prime}$. If $\mu=\mu^{\prime}$, then $\mathcal{B}$ outputs 1 . Otherwise, it outputs 0 .

If $Z=Z_{0}$, then the simulation is the same as $\mathbf{G}_{3}$ since the challenge ciphertext is correctly distributed by implicitly setting $s=b$. If $Z=Z_{1}$, then the simulation is the same as $\mathbf{G}_{4}$ since the element $C$ is random.

\subsubsection{Type-I Adversary}

Lemma 4.8. If the GSD assumption holds, then no polynomial-time Type-I adversary can distinguish between $\boldsymbol{H}_{\left(h_{m}, h_{c}-1\right), 2}$ and $\boldsymbol{H}_{\left(h_{m}, h_{c}\right), 1}$ with a non-negligible advantage.

Proof. Suppose there exists an adversary $\mathcal{A}_{I}$ that distinguishes between $\mathbf{H}_{\left(h_{m}, h_{c}-1\right), 2}$ and $\mathbf{H}_{\left(h_{m}, h_{c}\right), 1}$ with a non-negligible advantage. A simulator $\mathcal{B}$ that solves the GSD assumption using $\mathcal{A}_{I}$ is given: a challenge tuple $D=\left(\left(N, \mathbb{G}, \mathbb{G}_{T}, e\right), g_{p_{1}}, g_{p_{3}}, X_{1} R_{1}, R_{2} Y_{1}\right)$ and $Z$ where $Z=Z_{0}=X_{2} Y_{2}$ or $Z=Z_{1}=X_{2} R_{3} Y_{2}$. Then $\mathcal{B}$ that interacts with $\mathcal{A}_{I}$ is described as follows:

Setup: $\mathcal{B}$ selects random exponents $u_{1}^{\prime}, h_{1}^{\prime}, u_{2}^{\prime}, h_{2}^{\prime}, \alpha \in \mathbb{Z}_{N}$. It sets $\mathcal{B} \mathcal{T}$ by running SD.Setup and $F L$ by selecting $f_{G L}(x)$ for each $G L$ in $\mathcal{B T}$. It sets $M K=(\alpha, F L), R L=\emptyset, S T=(\mathcal{B T}, U L=\emptyset)$, and $P P=$ $\left(\left(N, \mathbb{G}, \mathbb{G}_{T}, e\right), g=g_{p_{1}}, Y=g_{p_{3}}, u_{1}=g^{u_{1}^{\prime}}, h_{1}=g^{h_{1}^{\prime}}, u_{2}=g^{u_{2}^{\prime}}, h_{2}=g^{h_{2}^{\prime}}, \Omega=e(g, g)^{\alpha}\right)$.

Phase 1: Let $h_{m}^{*}$ be a member index of the group index $h$ such that the personalized private key for $I D^{*}$ or the time-constrained update key for $T^{*}$ belong to the member index $h_{m}^{*}$ such that $1 \leq h_{m}^{*} \leq q_{m}$ where $q_{m}$ is the maximum number of members in the group index $h$. As mentioned before, there is only one index $h_{m}^{*}$ in the Type-I adversary. $\mathcal{B}$ selects a random index $k$ such that $1 \leq k \leq q_{m}$ to guess $h_{m}^{*}$, and it can correctly guess 
$h_{m}^{*}$ with the probability of $1 / q_{m}$. Note that $q_{m} \leq q_{s k}+q_{u k}$ since the private set of a private key is related with a path and the covering set of an update key is a partition where $q_{s k}$ is the number of private key queries and $q_{u k}$ is the number of update key queries of the adversary.

For each query, $\mathcal{B}$ proceeds as follows: If this is a decryption key query, then it creates a normal one since it knows $M K$. If this is a personalized private key or a time-constrained update key query with indexes $\left(i_{g}, i_{m}, i_{c}\right)$, then it handles this query as follows:

- Case $i_{g}<h$ : It first builds a normal key since it knows $M K$ and converts it to a semi-functional one by using $R_{2} Y_{1}$ that is given in the assumption and selecting a random exponent $\delta_{i, j}^{\prime}$ once for the subset $S_{i, j}$

- Case $i_{g}=h$ : It generates the key as follows:

- $\left(i_{m} \neq h_{m}^{*}\right) \wedge\left(i_{m}<h_{m}\right)$ or $\left(i_{m} \neq h_{m}^{*}\right) \wedge\left(i_{m}=h_{m}\right) \wedge\left(i_{c}<h_{c}\right)$ :

If this is a personalized private key query, then it first builds a normal one and converts it to a semi-functional one of type 2 by selecting a new random exponent $a^{\prime} \in \mathbb{Z}_{N}$ as $P S K_{I D, S_{i, j}}=\left(K_{0}=\right.$ $\left.g^{f_{G L}\left(L_{j}\right)}\left(u_{1}^{I D} h_{1}\right)^{r_{1}} Y_{0}^{\prime} \cdot\left(R_{2} Y_{1}\right)^{a^{\prime}}, K_{1}=g^{-r_{1}} Y_{1}^{\prime}\right)$.

If this is a time-constrained update key query, then it first builds a normal one and converts it to a semi-functional one of type 2 by selecting a new random exponent $a^{\prime} \in \mathbb{Z}_{N}$ as $T U K_{T, S_{i, j}}=$ $\left(U_{0}=g^{f_{G L}\left(L_{j}\right)}\left(u_{2}^{T} h_{2}\right)^{r_{2}} Y_{0}^{\prime} \cdot\left(R_{2} Y_{1}\right)^{a^{\prime}}, U_{1}=g^{-r_{2}} Y_{1}^{\prime}\right)$.

- $\left(i_{m} \neq h_{m}^{*}\right) \wedge\left(i_{m}=h_{m}\right) \wedge\left(i_{c}=h_{c}\right)$ :

If this is a personalized private key query, then it chooses random elements $Y_{0}^{\prime}, Y_{1}^{\prime} \in \mathbb{G}_{p_{3}}$ and creates a key as $P S K_{I D, S_{i, j}}=\left(K_{0}=g^{f_{G L}\left(L_{j}\right)}(Z)^{u_{1}^{\prime} I D+h_{1}^{\prime}} Y_{0}^{\prime}, K_{1}=Z^{-1} Y_{1}^{\prime}\right)$.

If this is a time-constrained update key query, then it chooses random elements $Y_{0}^{\prime}, Y_{1}^{\prime} \in \mathbb{G}_{p_{3}}$ and creates a key as $T U K_{T, S_{i, j}}=\left(U_{0}=g^{f_{G L}\left(L_{j}\right)}(Z)^{u_{2}^{\prime} T+h_{2}^{\prime}} Y_{0}^{\prime}, U_{2}=Z^{-1} Y_{1}^{\prime}\right)$.

- $\left(i_{m} \neq h_{m}^{*}\right) \wedge\left(i_{m}=h_{m}\right) \wedge\left(h_{c}<i_{c}\right)$ or $\left(i_{m} \neq h_{m}^{*}\right) \wedge\left(h_{m}<i_{m}\right)$ : It creates a normal key since it knows $M K$.

- $\left(i_{m}=h_{m}^{*}\right)$ : It creates a normal key since it knows $M K$.

- Case $i_{g}>h$ : It creates a normal key since it knows $M K$.

Challenge: $\mathcal{B}$ flips a random coin $\mu \in\{0,1\}$ and creates a semi-functional ciphertext by implicitly setting $g^{s}=X_{1}$ and $g_{2}^{c}=R_{1}$ as $C T^{*}=\left(C=e\left(X_{1} R_{1}, g\right)^{\alpha} \cdot M_{\mu}^{*}, C_{0}=X_{1} R_{1}, C_{1}=\left(X_{1} R_{1}\right)^{u_{1}^{\prime} I D^{*}+h_{1}^{\prime}}, C_{2}=\left(X_{1} R_{1}\right)^{u_{2}^{\prime} T^{*}+h_{2}^{\prime}}\right)$.

Phase 2: Same as Phase 1.

Guess: $\mathcal{A}$ outputs a guess $\mu^{\prime}$. If $\mu=\mu^{\prime}$, then $\mathcal{B}$ outputs 1 . Otherwise, it outputs 0 .

If $Z=Z_{0}=X_{2} Y_{2}$, then the simulation is the same as $\mathbf{H}_{\left(h_{m}, h_{c}-1\right), 2}$ since the personalized private key (or the time-constrained update key) with $\left(i_{m} \neq h_{m}^{*}\right) \wedge\left(i_{m}=h_{m}\right) \wedge\left(i_{c}=h_{c}\right)$ and the semi-functional challenge ciphertext are correctly distributed by implicitly setting $r_{1} \equiv \log _{g}\left(X_{2}\right) \bmod p_{1}\left(\operatorname{or} r_{2} \equiv \log _{g}\left(X_{2}\right) \bmod p_{1}\right)$, and $s \equiv \log _{g}\left(X_{1}\right) \bmod p_{1}$. If $Z=Z_{1}=X_{2} R_{3} Y_{2}$, then the simulation is almost the same as $\mathbf{H}_{\left(h_{m}, h_{c}\right), 1}$ except that the personalized private key (or the time-constrained update key) with $\left(i_{m} \neq h_{m}^{*}\right) \wedge\left(i_{m}=h_{m}\right) \wedge\left(i_{c}=h_{c}\right)$ is generated as a nominally semi-functional one of type 1 by implicitly setting $a \equiv \log _{g_{2}}\left(R_{3}\right)\left(u_{1}^{\prime} I D+h_{1}^{\prime}\right)$ $\bmod p_{2}\left(\right.$ or $\left.a \equiv \log _{g_{2}}\left(R_{3}\right)\left(u_{2}^{\prime} T+h_{2}^{\prime}\right) \bmod p_{2}\right)$ and $b \equiv \log _{g_{2}}\left(R_{3}\right) \bmod p_{2}$. Note that we solve the paradox of the dual system encryption by introducing the nominally semi-functional one of type 1 which is similar to that of Lewko and Waters [25]. That is, the simulator cannot distinguish whether the personalized private key (or time-constrained update key) of $h_{c}$ is normal or nominally semi-functional since the nominally semifunctional one is correlated to the challenge ciphertext. 
To finish the proof, we should argue that the Type-I adversary cannot distinguish the nominally semifunctional one of type 1 from the semi-functional one of type 1. For this argument, we show an information theoretic argument by using the fact that we have $I D \neq I D^{*}$ for all personalized private key queries for $I D$ with indexes $\left(i_{g}=h, i_{m}, i_{c}\right)$ such that $i_{m} \neq h_{m}^{*}$, and $T \neq T^{*}$ for all time-constrained update key queries for $T$ with indexes $\left(i_{g}=h, i_{m}, i_{c}\right)$ such that $i_{m} \neq h_{m}^{*}$. Suppose there exists an unbounded Type-I adversary. If the query with $\left(i_{m} \neq h_{m}^{*}\right) \wedge\left(i_{m}=h_{m}\right) \wedge\left(i_{c}=h_{c}\right)$ is a personalized private key, then the adversary can gather the values $a \equiv b\left(u_{1}^{\prime} I D+h_{1}^{\prime}\right), b \bmod p_{2}$ from the personalized private key with $\left(i_{m} \neq h_{m}^{*}\right) \wedge\left(i_{m}=\right.$ $\left.h_{m}\right) \wedge\left(i_{c}=h_{c}\right)$ and $d_{1} \equiv u_{1}^{\prime} I D^{*}+h_{1}^{\prime} \bmod p_{2}, d_{2} \equiv u_{2}^{\prime} T^{*}+h_{2}^{\prime} \bmod p_{2}$ from the challenge ciphertext. We obtain that $u_{1}^{\prime} I D+h_{1}^{\prime} \bmod p_{2}$ and $u_{1}^{\prime} I D^{*}+h_{1}^{\prime} \bmod p_{2}$ look random to the adversary since $u_{1}^{\prime} x+h_{1}^{\prime}$ is a pairwise independent function, $I D \neq I D^{*}$ if $i_{m} \neq h_{m}^{*}$, and $u_{1}^{\prime}, h_{1}^{\prime} \bmod p_{2}$ are information theoretically hidden to the adversary. If the query with $\left(i_{m} \neq h_{m}^{*}\right) \wedge\left(i_{m}=h_{m}\right) \wedge\left(i_{c}=h_{c}\right)$ is a time-constrained update key, then we also obtain that $u_{2}^{\prime} T+h_{2}^{\prime} \bmod p_{2}$ and $u_{2}^{\prime} T^{*}+h_{2}^{\prime}$ are random to the adversary since $u_{2}^{\prime} x+h_{2}^{\prime}$ pair-wise independent function, $T \neq T^{*}$ if $i_{m} \neq h_{m}^{*}$, and $u_{2}^{\prime}, h_{2}^{\prime} \bmod p_{2}$ are information theoretically hidden to the adversary. This completes our proof.

Lemma 4.9. If the GSD assumption holds, then no polynomial-time Type-I adversary can distinguish between $\boldsymbol{H}_{\left(h_{m}, h_{c}\right), 1}$ and $\boldsymbol{H}_{\left(h_{m}, h_{c}\right), 2}$ with a non-negligible advantage.

Proof. The proof of this lemma is almost the same as that of Lemma 4.8 except the generation of the key with indexes $i_{g}=h$ and $\left(i_{m} \neq h_{m}^{*}\right) \wedge\left(i_{m}=h_{m}\right) \wedge\left(i_{c}=h_{c}\right)$. This key with the group index $h$ is generated as follows:

- $\left(i_{m} \neq h_{m}^{*}\right) \wedge\left(i_{m}=h_{m}\right) \wedge\left(i_{c}=h_{c}\right)$ :

If this is a personalized private key query, then it chooses random $a^{\prime} \in \mathbb{Z}_{N}, Y_{0}^{\prime}, Y_{1}^{\prime} \in \mathbb{G}_{p_{3}}$ and creates a key as $P S K_{I D, S_{i, j}}=\left(K_{0}=g^{f_{G L}\left(L_{j}\right)}(Z)^{u_{1}^{\prime} I D+h_{1}^{\prime}} Y_{0}^{\prime}\left(R_{2} Y_{1}\right)^{a^{\prime}}, K_{1}=Z^{-1} Y_{1}^{\prime}\right)$.

If this is a time-constrained update key query, then it chooses random $a^{\prime} \in \mathbb{Z}_{N}, Y_{0}^{\prime}, Y_{1}^{\prime} \in \mathbb{G}_{p_{3}}$ and creates a key as $T U K_{T, S_{i, j}}=\left(U_{0}=g^{f_{G L}\left(L_{j}\right)}(Z)^{u_{2}^{\prime} T+h_{2}^{\prime}} Y_{0}^{\prime}\left(R_{2} Y_{1}\right)^{a^{\prime}}, K_{1}=Z^{-1} Y_{1}^{\prime}\right)$.

Note that this personalized private key or time-constrained update key is no longer correlated with $C T^{*}$ since the element $K_{0}$ is re-randomized by $\left(R_{2} Y_{1}\right)^{a^{\prime}}$.

Lemma 4.10. If the GSD assumption holds, then no polynomial-time Type-I adversary can distinguish between $\boldsymbol{H}_{\left(h_{m}, h_{c}-1\right), 2}^{\prime}$ and $\boldsymbol{H}_{\left(h_{m}, h_{c}\right), 1}^{\prime}$ with a non-negligible advantage.

Lemma 4.11. If the GSD assumption holds, then no polynomial-time Type-I adversary can distinguish between $\boldsymbol{H}_{\left(h_{m}, h_{c}\right), 1}^{\prime}$ and $\boldsymbol{H}_{\left(h_{m}, h_{c}\right), 2}^{\prime}$ with a non-negligible advantage.

The proofs of Lemma 4.10 and Lemma 4.11 are almost the same as that of Lemma 4.8 and Lemma 4.9 respectively. The only difference is that each element $K_{0}$ of personalized private keys and each element $U_{0}$ of time-constrained update keys with indexes $\left(i_{g}=h, i_{m}, i_{c}\right)$ such that $i_{m} \neq h_{m}^{*}$ that are generated in Lemma 4.8 and Lemma 4.9 respectively are additionally multiplied by $\left(R_{2} Y_{1}\right)^{\delta_{i, j}^{\prime}}$ where $\delta_{i, j}^{\prime}$ is a fixed exponent that is related with the member subset $S_{i, j}$. This modification is possible since $R_{2} Y_{1}$ is given in the assumption. We omit the detailed proofs of these lemmas.

Lemma 4.12. If the GSD assumption holds, then no polynomial-time Type-I adversary can distinguish between $\boldsymbol{H}_{(1,0), 2}^{\prime}$ and $\boldsymbol{H}^{\prime \prime}$ with a non-negligible advantage. 
Proof. The proof of this lemma is the core of the RIBE security proof since it changes the personalized private key for $I D^{*}$ and the time-constrained update key for $T^{*}$ from normal type to semi-functional type. It should be noted that this changes from normal to semi-functional cannot be handled by introducing nominally semi-functional type since an information theoretic argument for $I D^{*}$ and $T^{*}$ cannot be used. Recall that $h_{m}^{*}$ be the member index that is related to $I D^{*}$ and $T^{*}$. To solve this problem, we directly change normal keys for $h_{m}^{*}$ to semi-functional keys without introducing nominally semi-functional keys, and then we argue that the paradox of dual system encryption can be solved by the property of the Lagrange interpolation method.

Many parts of this proof is similar to that of Lemma 4.8 except the generation of personalized private keys and time-constrained update keys with the group index $i_{g}=h$. These keys with the group index $i_{g}=h$ are generated as follows:

- Case $i_{g}=h$ : Let $\delta_{i, j}^{\prime}$ be a fixed exponent in $\mathbb{Z}_{N}$ for each member $S_{i, j}$ in this group index $h$.

$-\left(i_{m} \neq h_{m}^{*}\right)$ :

If this is a personalized private key query, then it selects random $r_{1} \in \mathbb{Z}_{N}, Y_{0}^{\prime}, Y_{1}^{\prime} \in \mathbb{G}_{p_{3}}$ and creates a semi-functional key as $P S K_{I D, S_{i, j}}=\left(K_{0}=(Z)^{L_{j}} g^{\alpha}\left(u_{1}^{I D} h_{1}\right)^{r_{1}} Y_{0}^{\prime} \cdot\left(R_{2} Y_{1}\right)^{\delta_{i, j}^{\prime}}, K_{1}=g^{-r_{1}} Y_{1}^{\prime}\right)$.

If this is a time-constrained update key query, then it selects random $r_{2} \in \mathbb{Z}_{N}, Y_{0}^{\prime}, Y_{1}^{\prime} \in \mathbb{G}_{p_{3}}$ and creates a semi-functional key as $T U K_{T, S_{i, j}}=\left(U_{0}=(Z)^{L_{j}} g^{\alpha}\left(u_{2}^{T} h_{2}\right)^{r_{2}} Y_{0}^{\prime} \cdot\left(R_{2} Y_{1}\right)^{\delta_{i, j}^{\prime}}, U_{1}=g^{-r_{2}} Y_{1}^{\prime}\right)$.

$-\left(i_{m}=h_{m}^{*}\right)$ :

If this is a personalized private key query, then it selects random $r_{1} \in \mathbb{Z}_{N}, Y_{0}^{\prime}, Y_{1}^{\prime} \in \mathbb{G}_{p_{3}}$ and creates a key as $P S K_{I D, S_{i, j}}=\left(K_{0}=(Z)^{L_{j}} g^{\alpha}\left(u_{1}^{I D} h_{1}\right)^{r_{1}} Y_{0}^{\prime}, K_{1}=g^{-r_{1}} Y_{1}^{\prime}\right)$.

If this is a time-constrained update key query, then it selects random $r_{2} \in \mathbb{Z}_{N}, Y_{0}^{\prime}, Y_{1}^{\prime} \in \mathbb{G}_{p_{3}}$ and creates a key as $T U K_{T, S_{i, j}}=\left(U_{0}=(Z)^{L_{j}} g^{\alpha}\left(u_{2}^{T} h_{2}\right)^{r_{2}} Y_{0}^{\prime}, U_{1}=g^{-r_{2}} Y_{1}^{\prime}\right)$.

If $Z=Z_{0}=X_{2} Y_{2}$, then the simulation is the same as $\mathbf{H}_{(1,0), 2}^{\prime}$ since all personalized private keys and timeconstrained update keys with the group index $h$ implicitly uses a random polynomial $f_{G L}(x) \equiv \log _{g}\left(X_{2}\right)$. $x+\alpha \bmod p_{1}$ and it implicitly sets $\delta_{i, j} \equiv \log _{g_{p_{2}}}\left(R_{2}\right) \delta_{i, j}^{\prime} \bmod p_{2}$ for each member index $i_{m} \neq h_{m}^{*}$. If $Z=$ $Z_{1}=X_{2} R_{3} Y_{2}$, then the simulation is the same as $\mathbf{H}^{\prime \prime}$ since it implicitly sets $\delta_{i, j}=\log _{g_{p_{2}}}\left(R_{3}\right) L_{j} \bmod p_{2}$ for the member index $h_{m}^{*}$. As mentioned before, the personalized private key query for $I D^{*}$ and the timeconstrained update key query for $T^{*}$ should belong to the same member index $h_{m}^{*}$ by the restriction $I D^{*} \in R^{*}$ of the security model.

We now argue that the paradox of dual system encryption can be solved. To distinguish whether a personalized private key for $h_{m}^{*}$ and a time-constrained update key for $h_{m}^{*}$ are normal or semi-functional, the simulator may try to decrypt a semi-functional ciphertext by deriving a decryption key from these keys for $h_{m}^{*}$. However, the simulator cannot derive a decryption key from those keys since the Lagrange interpolation method does not work for the same $h_{m}^{*}$ since only one point of $f_{G L}(x)$ is revealed. Recall that the Lagrange interpolation method requires two points of $f_{G L}(x)$ to derive $f_{G L}(0)$. Thus, the simulator cannot distinguish whether these two keys for the same $h_{m}^{*}$ are normal or semi-functional. This completes our proof.

\subsubsection{Type-II Adversary}

Lemma 4.13. If the GSD assumption holds, then no polynomial-time Type-II adversary can distinguish between $\boldsymbol{I}_{\left(h_{m}, h_{c}-1\right), 2}$ and $\boldsymbol{I}_{\left(h_{m}, h_{c}\right), 1}$ with a non-negligible advantage.

Lemma 4.14. If the GSD assumption holds, then no polynomial-time Type-II adversary can distinguish between $\boldsymbol{I}_{\left(h_{m}, h_{c}\right), 1}$ and $\boldsymbol{I}_{\left(h_{m}, h_{c}\right), 2}$ with a non-negligible advantage. 
Lemma 4.15. If the GSD assumption holds, then no polynomial-time Type-II adversary can distinguish between $\boldsymbol{I}_{\left(h_{m}, h_{c}-1\right), 2}^{\prime}$ and $\boldsymbol{I}_{\left(h_{m}, h_{c}\right), 1}^{\prime}$ with a non-negligible advantage.

Lemma 4.16. If the GSD assumption holds, then no polynomial-time Type-II adversary can distinguish between $\boldsymbol{I}_{\left(h_{m}, h_{c}\right), 1}^{\prime}$ and $\boldsymbol{I}_{\left(h_{m}, h_{c}\right), 2}^{\prime}$ with a non-negligible advantage.

The proofs of Lemmas 4.13, 4.14, 4.15, and 4.16 are almost the same as those of Lemmas 4.8, 4.9, 4.10, and 4.11 respectively except that there is no case $i_{m}=h_{m}^{*}$ since the Type-II adversary does not request a personalized private key for $I D^{*}$ and a time-constrained update key for $T^{*}$. We omit the detailed proofs of these lemmas.

\subsection{Discussions}

Prime-Order Groups Our RIBE scheme is fully secure in composite-order bilinear groups under static assumptions. To improve the efficiency of our RIBE scheme, we may consider to use prime-order bilinear groups instead of composite-order bilinear groups. A general methodology that converts cryptosystems that use dual system encryption techniques in composite-order bilinear groups into prime-order setting was introduced by Lewko [22]. Lewko showed that the parameter hiding property of composite-order bilinear groups can be achieved in prime-order groups by using the dual pairing vector space proposed by Okamoto and Takashima [33]. We expect that our RIBE scheme in composite-order bilinear groups also can be converted into prime-order setting by slightly modifying the prime-order IBE scheme of Lewko.

\section{Conclusion}

In this work, we presented a new technique for RIBE that can use the efficient SD scheme (or the LSD scheme) instead of the CS scheme for key revocation. By following our technique, we first proposed a new RIBE scheme in bilinear groups by combining the IBE scheme of Boneh and Boyen [5] and the SD scheme, and then we proved its security in the selective revocation list model. We also proposed another RIBE scheme in bilinear groups by combining the IBE scheme in composite-order bilinear groups of Lewko and Waters [25] and the SD scheme, and proved its full security under static assumptions. Our constructions also can be integrated with the LSD scheme to reduce the size of private keys.

An interesting open problem is to build efficient R-ABE, RS-ABE, and RS-PE schemes that provide the revocation functionality for $\mathrm{ABE}$ and $\mathrm{PE}$ by using the SD scheme. One may expect that our technique in this work can be used to achieve these schemes, but there is one crucial difficulty to prove the security of the schemes since our proof techniques in the selective revocation list model (or the full model) only work when there is only one private key (or one update key) that matches to a challenge ciphertext. However, there are many private key queries in $\mathrm{ABE}$ that can decrypt a challenge ciphertext. Thus, we expect that a new different technique will be needed to build R-ABE, RS-ABE, and RS-PE schemes that use the SD scheme.

\section{References}

[1] William Aiello, Sachin Lodha, and Rafail Ostrovsky. Fast digital identity revocation (extended abstract). In Hugo Krawczyk, editor, CRYPTO '98, volume 1462 of Lecture Notes in Computer Science, pages 137-152. Springer, 1998. 
[2] Nuttapong Attrapadung and Hideki Imai. Conjunctive broadcast and attribute-based encryption. In Hovav Shacham and Brent Waters, editors, Pairing 2009, volume 5671 of Lecture Notes in Computer Science, pages 248-265. Springer, 2009.

[3] John Bethencourt, Amit Sahai, and Brent Waters. Ciphertext-policy attribute-based encryption. In IEEE Symposium on Security and Privacy, pages 321-334. IEEE Computer Society, 2007.

[4] Alexandra Boldyreva, Vipul Goyal, and Virendra Kumar. Identity-based encryption with efficient revocation. In Peng Ning, Paul F. Syverson, and Somesh Jha, editors, ACM Conference on Computer and Communications Security, pages 417-426. ACM, 2008.

[5] Dan Boneh and Xavier Boyen. Efficient selective-id secure identity-based encryption without random oracles. In Christian Cachin and Jan Camenisch, editors, EUROCRYPT 2004, volume 3027 of Lecture Notes in Computer Science, pages 223-238. Springer, 2004.

[6] Dan Boneh and Matthew K. Franklin. Identity-based encryption from the weil pairing. In Joe Kilian, editor, CRYPTO 2001, volume 2139 of Lecture Notes in Computer Science, pages 213-229. Springer, 2001.

[7] Dan Boneh, Amit Sahai, and Brent Waters. Functional encryption: Definitions and challenges. In Yuval Ishai, editor, TCC 2011, volume 6597 of Lecture Notes in Computer Science, pages 253-273. Springer, 2011.

[8] Dan Boneh and Brent Waters. Conjunctive, subset, and range queries on encrypted data. In Salil P. Vadhan, editor, TCC 2007, volume 4392 of Lecture Notes in Computer Science, pages 535-554. Springer, 2007.

[9] Ran Canetti, Shai Halevi, and Jonathan Katz. Chosen-ciphertext security from identity-based encryption. In Christian Cachin and Jan Camenisch, editors, EUROCRYPT 2004, volume 3027 of Lecture Notes in Computer Science, pages 207-222. Springer, 2004.

[10] Amos Fiat and Moni Naor. Broadcast encryption. In Douglas R. Stinson, editor, CRYPTO '93, volume 773 of Lecture Notes in Computer Science, pages 480-491. Springer, 1993.

[11] Craig Gentry. Certificate-based encryption and the certificate revocation problem. In Eli Biham, editor, EUROCRYPT 2003, volume 2656 of Lecture Notes in Computer Science, pages 272-293. Springer, 2003.

[12] Craig Gentry and Alice Silverberg. Hierarchical id-based cryptography. In Yuliang Zheng, editor, ASIACRYPT 2002, volume 2501 of Lecture Notes in Computer Science, pages 548-566. Springer, 2002.

[13] Sergey Gorbunov, Vinod Vaikuntanathan, and Hoeteck Wee. Functional encryption with bounded collusions via multi-party computation. In Reihaneh Safavi-Naini and Ran Canetti, editors, CRYPTO 2012, volume 7417 of Lecture Notes in Computer Science, pages 162-179. Springer, 2012.

[14] Vipul Goyal, Omkant Pandey, Amit Sahai, and Brent Waters. Attribute-based encryption for finegrained access control of encrypted data. In Ari Juels, Rebecca N. Wright, and Sabrina De Capitani di Vimercati, editors, ACM Conference on Computer and Communications Security, pages 89-98. ACM, 2006. 
[15] Dani Halevy and Adi Shamir. The lsd broadcast encryption scheme. In Moti Yung, editor, CRYPTO 2002, volume 2442 of Lecture Notes in Computer Science, pages 47-60. Springer, 2002.

[16] Jeremy Horwitz and Ben Lynn. Toward hierarchical identity-based encryption. In Lars R. Knudsen, editor, EUROCRYPT 2002, volume 2332 of Lecture Notes in Computer Science, pages 466-481. Springer, 2002.

[17] Jonathan Katz, Amit Sahai, and Brent Waters. Predicate encryption supporting disjunctions, polynomial equations, and inner products. In Nigel P. Smart, editor, EUROCRYPT 2008, volume 4965 of Lecture Notes in Computer Science, pages 146-162. Springer, 2008.

[18] Kwangsu Lee, Seung Geol Choi, Dong Hoon Lee, Jong Hwan Park, and Moti Yung. Self-updatable encryption: Time constrained access control with hidden attributes and better efficiency. In Kazue Sako and Palash Sarkar, editors, ASIACRYPT 2013, volume 8269 of Lecture Notes in Computer Science, pages 235-254. Springer, 2013.

[19] Kwangsu Lee, Intae Kim, and Seong Oun Hwang. Privacy preserving revocable predicate encryption revisited. Secur. Commun. Netw., 8(3):471-485, 2015.

[20] Kwangsu Lee, Woo Kwon Koo, Dong Hoon Lee, and Jong Hwan Park. Public-key revocation and tracing schemes with subset difference methods revisited. In Miroslaw Kutylowski and Jaideep Vaidya, editors, ESORICS 2014, volume 8713 of Lecture Notes in Computer Science, pages 1-18. Springer, 2014.

[21] Kwangsu Lee and Dong Hoon Lee. Improved hidden vector encryption with short ciphertexts and tokens. Designs Codes Cryptogr., 58(3):297-319, 2011.

[22] Allison B. Lewko. Tools for simulating features of composite order bilinear groups in the prime order setting. In David Pointcheval and Thomas Johansson, editors, EUROCRYPT 2012, volume 7237 of Lecture Notes in Computer Science, pages 318-335. Springer, 2012.

[23] Allison B. Lewko, Tatsuaki Okamoto, Amit Sahai, Katsuyuki Takashima, and Brent Waters. Fully secure functional encryption: Attribute-based encryption and (hierarchical) inner product encryption. In Henri Gilbert, editor, EUROCRYPT 2010, volume 6110 of Lecture Notes in Computer Science, pages 62-91. Springer, 2010.

[24] Allison B. Lewko, Amit Sahai, and Brent Waters. Revocation systems with very small private keys. In IEEE Symposium on Security and Privacy, pages 273-285. IEEE Computer Society, 2010.

[25] Allison B. Lewko and Brent Waters. New techniques for dual system encryption and fully secure hibe with short ciphertexts. In Daniele Micciancio, editor, TCC 2010, volume 5978 of Lecture Notes in Computer Science, pages 455-479. Springer, 2010.

[26] Benoît Libert and Damien Vergnaud. Adaptive-id secure revocable identity-based encryption. In Marc Fischlin, editor, CT-RSA 2009, volume 5473 of Lecture Notes in Computer Science, pages 1-15. Springer, 2009.

[27] Thomas Martin, Keith M. Martin, and Peter R. Wild. Establishing the broadcast efficiency of the subset difference revocation scheme. Designs Codes Cryptogr., 51(3):315-334, 2009. 
[28] Silvio Micali. Efficient certificate revocation. Technical Report TM-542b, MIT Laboratory for Computer Science, 1996.

[29] Dalit Naor, Moni Naor, and Jeffery Lotspiech. Revocation and tracing schemes for stateless receivers. In Joe Kilian, editor, CRYPTO 2001, volume 2139 of Lecture Notes in Computer Science, pages 41-62. Springer, 2001.

[30] Moni Naor and Kobbi Nissim. Certificate revocation and certificate update. IEEE J. Sel. Areas Commun., 18(4):561-570, 2000.

[31] Moni Naor and Benny Pinkas. Efficient trace and revoke schemes. In Yair Frankel, editor, FC 2000, volume 1962 of Lecture Notes in Computer Science, pages 1-20. Springer, 2000.

[32] Juan Manuel González Nieto, Mark Manulis, and Dongdong Sun. Fully private revocable predicate encryption. In Willy Susilo, Yi Mu, and Jennifer Seberry, editors, ACISP 2012, volume 7372 of Lecture Notes in Computer Science, pages 350-363. Springer, 2012.

[33] Tatsuaki Okamoto and Katsuyuki Takashima. Hierarchical predicate encryption for inner-products. In Mitsuru Matsui, editor, ASIACRYPT 2009, volume 5912 of Lecture Notes in Computer Science, pages 214-231. Springer, 2009.

[34] Tatsuaki Okamoto and Katsuyuki Takashima. Adaptively attribute-hiding (hierarchical) inner product encryption. In David Pointcheval and Thomas Johansson, editors, EUROCRYPT 2012, volume 7237 of Lecture Notes in Computer Science, pages 591-608. Springer, 2012.

[35] Seunghwan Park, Kwangsu Lee, and Dong Hoon Lee. New constructions of revocable identity-based encryption from multilinear maps. IEEE Trans. Inf. Forensic Secur., 10(8):1564-1577, 2015.

[36] Amit Sahai, Hakan Seyalioglu, and Brent Waters. Dynamic credentials and ciphertext delegation for attribute-based encryption. In Reihaneh Safavi-Naini and Ran Canetti, editors, CRYPTO 2012, volume 7417 of Lecture Notes in Computer Science, pages 199-217. Springer, 2012.

[37] Amit Sahai and Brent Waters. Fuzzy identity-based encryption. In Ronald Cramer, editor, EUROCRYPT 2005, volume 3494 of Lecture Notes in Computer Science, pages 457-473. Springer, 2005.

[38] Jae Hong Seo and Keita Emura. Efficient delegation of key generation and revocation functionalities in identity-based encryption. In Ed Dawson, editor, CT-RSA 2013, volume 7779 of Lecture Notes in Computer Science, pages 343-358. Springer, 2013.

[39] Jae Hong Seo and Keita Emura. Revocable identity-based encryption revisited: Security model and construction. In Kaoru Kurosawa and Goichiro Hanaoka, editors, PKC 2013, volume 7778 of Lecture Notes in Computer Science, pages 216-234. Springer, 2013.

[40] Adi Shamir. Identity-based cryptosystems and signature schemes. In G. R. Blakley and David Chaum, editors, CRYPTO '84, volume 196 of Lecture Notes in Computer Science, pages 47-53. Springer, 1984.

[41] Le Su, Hoon Wei Lim, San Ling, and Huaxiong Wang. Revocable ibe systems with almost constantsize key update. In Zhenfu Cao and Fangguo Zhang, editors, Pairing 2013, volume 8365 of Lecture Notes in Computer Science, pages 168-185. Springer, 2013. 
[42] Brent Waters. Efficient identity-based encryption without random oracles. In Ronald Cramer, editor, EUROCRYPT 2005, volume 3494 of Lecture Notes in Computer Science, pages 114-127. Springer, 2005 .

[43] Brent Waters. Dual system encryption: Realizing fully secure ibe and hibe under simple assumptions. In Shai Halevi, editor, CRYPTO 2009, volume 5677 of Lecture Notes in Computer Science, pages 619-636. Springer, 2009. 